\title{
Search for phenomena beyond the Standard Model in events with large $b$-jet multiplicity using the ATLAS detector at the LHC
}

\author{
ATLAS Collaboration ${ }^{\star}$ \\ CERN, 1211 Geneva 23, Switzerland
}

Received: 2 October 2020 / Accepted: 4 December 2020 / Published online: 11 January 2021

(C) CERN for the benefit of the ATLAS collaboration 2021, corrected publication 2021

\begin{abstract}
A search is presented for new phenomena in events characterised by high jet multiplicity, no leptons (electrons or muons), and four or more jets originating from the fragmentation of $b$-quarks ( $b$-jets). The search uses $139 \mathrm{fb}^{-1}$ of $\sqrt{s}=13 \mathrm{TeV}$ proton-proton collision data collected by the ATLAS experiment at the Large Hadron Collider during Run 2. The dominant Standard Model background originates from multijet production and is estimated using a datadriven technique based on an extrapolation from events with low $b$-jet multiplicity to the high $b$-jet multiplicities used in the search. No significant excess over the Standard Model expectation is observed and $95 \%$ confidence-level limits that constrain simplified models of R-parity-violating supersymmetry are determined. The exclusion limits reach $950 \mathrm{GeV}$ in top-squark mass in the models considered.
\end{abstract}

\section{Introduction}

Events with a large number of high-transverse momentum $\left(p_{\mathrm{T}}\right)$ jets originating from the fragmentation of $b$-quarks $(b-$ jets) are rarely produced by Standard Model (SM) processes in proton-proton $(p p)$ collisions at the LHC. As a result, this signature can provide sensitivity to certain phenomena beyond the SM (BSM) [1-3]. Event signatures with five or more $b$-jets, no leptons (electrons or muons) and without any requirements on missing transverse momentum are not covered by existing searches at the LHC.

Supersymmetry (SUSY) provides an extension to the SM by introducing partners of the known bosons and fermions. It predicts the existence of superpartner states (with different statistics) associated to each of the SM particles and fields. The lightest among such superpartners (LSP) may or may not be stable, depending on the conservation of R-parity [46]. Final states with high leptonic or hadronic multiplicity are commonly predicted by R-parity-violating (RPV) SUSY. Models of RPV SUSY do not provide stable superpartners, and they give rise to a wide variety of experimental signatures

^e-mail: atlas.publications@cern.ch whose nature depends on which of the many RPV couplings are non-zero.

In the analysis presented here, a particular benchmark model is considered in order to interpret the measurements in the different jet and $b$-jet multiplicity regions. The process under consideration is the pair production of the top squark as the lightest of the coloured SUSY partners. The existence of light SUSY partners of third-generation quarks, bottom squarks $(\tilde{b})$ and top squarks $(\tilde{t})$, is favoured by naturalness considerations $[7,8]$. The scenario assumes the LSP to be a triplet of two neutralino $\left(\tilde{\chi}_{1}^{0}, \tilde{\chi}_{2}^{0}\right)$ and one chargino $\left(\tilde{\chi}_{1}^{ \pm}\right)$states that are mass-degenerate and carry dominantly higgsino components (in the following collectively referred to as "higgsinos"). The top squark decays either into a chargino, $\tilde{\chi}_{1}^{ \pm}$, and a bottom quark or into a neutralino, $\tilde{\chi}_{1,2}^{0}$, and a top quark. The chargino and neutralino decay, respectively, to $b b s$ and $t b s$ quark triplets, as shown in Fig. 1; this decay is mediated through their higgsino components via the non-zero baryon-number-violating RPV coupling $\lambda_{323}^{\prime \prime}$ $[9,10]$.

When $m_{\tilde{t}}-m_{\tilde{\chi}_{1,2}^{0}, \tilde{x}_{1}^{ \pm}} \leq m_{\text {top }}$ (Fig. 1a), the $\tilde{t} \rightarrow t \tilde{\chi}_{1,2}^{0}$ decay is kinematically forbidden and the top-squark branching ratio (B) to $b \tilde{\chi}_{1}^{ \pm}$is equal to unity; when $m_{\tilde{t}}-m_{\tilde{\chi}_{1,2}^{0}, \tilde{\chi}_{1}^{ \pm}} \geq m_{\text {top }}$ the value of $B$ is taken to be 0.5 . In the latter case, the rest of the decay rate is evenly divided between the two neutralino states: $\tilde{t} \rightarrow t \tilde{\chi}_{1,2}^{0}\left(\tilde{\chi}_{1,2}^{0} \rightarrow t b s\right)$ (Fig. 1b). For the supersymmetric particle masses under consideration, the analysis considers only values of $\lambda_{323}^{\prime \prime} \approx O\left(10^{-2}-10^{-1}\right)$ [11] which ensure prompt neutralino and chargino decays and omit more complex RPV decay patterns such as $\tilde{\chi}_{1}^{ \pm} \rightarrow W^{ \pm *} \tilde{\chi}_{1}^{0}\left(\tilde{\chi}_{1}^{0} \rightarrow\right.$ $t b s)$ or $\tilde{\chi}_{2}^{0} \rightarrow Z^{*} \tilde{\chi}_{1}^{0}\left(\tilde{\chi}_{1}^{0} \rightarrow t b s\right)$ that could be substantial for very small values of $\lambda_{323}^{\prime \prime}$ [3].

Previous searches targeting RPV SUSY models of pairproduced top squarks decaying through the coupling $\lambda_{323}^{\prime \prime}$ have been carried out by the ATLAS and CMS collaborations. Those searches already exclude top-squark masses in the ranges $100 \mathrm{GeV} \leq m_{\tilde{t}} \leq 470 \mathrm{GeV}$ and $480 \mathrm{GeV} \leq m_{\tilde{t}} \leq$ $610 \mathrm{GeV}$ (ATLAS [12]), and $80 \mathrm{GeV} \leq m_{\tilde{t}} \leq 270 \mathrm{GeV}$, $285 \mathrm{GeV} \leq m_{\tilde{t}} \leq 340 \mathrm{GeV}$ and $400 \mathrm{GeV} \leq m_{\tilde{t}} \leq 505 \mathrm{GeV}$ 
(CMS [13]) in scenarios where the top squark is the LSP and decays directly via $\tilde{t} \rightarrow b s$. For the direct top-squark production and $\lambda_{323}^{\prime \prime}$-mediated decays of higgsino LSP scenarios, ATLAS has excluded top-squark masses up to $1.10 \mathrm{TeV}$, depending on the higgsino mass considered, in the region where $m_{\tilde{t}}-m_{\tilde{\chi}_{1,2}^{0}, \tilde{\chi}_{1}^{ \pm}} \geq m_{\text {top }}$, by analysing lepton plus jets events [11]. CMS has excluded top-squark masses between 100 and $720 \mathrm{GeV}$ for top-squark decays into four quarks in boosted topologies and with the mass of the higgsinos set to $75 \%$ of the squark mass [14].

This analysis considers events with six or more jets, of which at least four are identified as $b$-jets ( $b$-tagged). There must be no identified electron or muon, and no requirement is made on the missing transverse momentum. In this channel, the dominant background is the non-resonant production of multijet events, referred to as 'multijet' in the following, and a data-driven method is applied to estimate its yield. Other backgrounds arise from top-quark pair production accompanied by extra $b$-jets or by a $Z$ or Higgs boson decaying into a $b$-quark pair. Results are reported as $95 \%$ confidence level (CL) exclusion limits on the top-squark mass in the benchmark models described above. Modelindependent limits on the possible contribution of BSM physics are also evaluated at large jet and $b$-tagged jet multiplicities.

\section{ATLAS detector}

The ATLAS experiment [15] at the LHC is a multipurpose particle detector with a forward-backward symmetric cylindrical geometry and a near $4 \pi$ coverage in solid angle. ${ }^{1}$ It consists of an inner tracking detector (ID) surrounded by a thin superconducting solenoid providing a $2 \mathrm{~T}$ axial magnetic field, electromagnetic and hadron calorimeters, and a muon spectrometer (MS). The inner tracking detector covers the pseudorapidity range $|\eta|<2.5$. It consists of silicon pixel, silicon microstrip, and transition radiation tracking detectors. An additional innermost layer of the silicon pixel tracker, the insertable B-layer [16, 17], was installed in 2014 at an average radial distance of $3.3 \mathrm{~cm}$ from the beam-line to improve track reconstruction and flavour identification of quark-initiated jets. Lead/liquid-argon (LAr) sampling calorimeters provide electromagnetic energy measurements with high granularity. A steel/scintillator-tile calorimeter provides hadronic energy

\footnotetext{
1 ATLAS uses a right-handed coordinate system with its origin at the nominal interaction point (IP) in the centre of the detector and the $z$ axis along the beam pipe. The $x$-axis points from the IP to the centre of the LHC ring, and the $y$-axis points upwards. Cylindrical coordinates $(r, \phi)$ are used in the transverse plane, $\phi$ being the azimuthal angle around the $z$-axis. The pseudorapidity is defined in terms of the polar angle $\theta$ as $\eta=-\ln \tan (\theta / 2)$. Angular distance is measured in units of $\Delta R \equiv \sqrt{(\Delta \eta)^{2}+(\Delta \phi)^{2}}$.
}

measurements and covers the central pseudorapidity range $(|\eta|<1.7)$. The endcap and forward regions are instrumented with LAr calorimeters for both the electromagnetic and hadronic energy measurements up to $|\eta|=4.9$. The muon spectrometer surrounds the calorimeters and is based on three large air-core toroidal superconducting magnets with eight coils each. The field integral of the toroids ranges between 2.0 and $6.0 \mathrm{~T} \mathrm{~m}$ across most of the detector. The muon spectrometer includes a system of precision tracking chambers and fast detectors for triggering. A two-level trigger system is used to select events to be recorded. The firstlevel trigger is implemented in hardware and uses a subset of the detector information to accept events at a rate of at most $100 \mathrm{kHz}$. This is followed by a software-based high-level trigger (HLT) that reduces the accepted event rate to $\sim 1.2$ $\mathrm{kHz}$, on average.

\section{Data collection and simulated event samples}

This search is based on $139 \mathrm{fb}^{-1}$ of centre-of-mass energy $\sqrt{s}=13 \mathrm{TeV} p p$ collision data, collected between 2015 and 2018 , that satisfy beam, detector and data-quality criteria. The uncertainty in the combined 2015-2018 integrated luminosity is $1.7 \%$ [18], obtained using the LUCID-2 detector [19] for the primary luminosity measurements. The average number of interactions $(\langle\mu\rangle)$ in the same and nearby bunch crossings (pile-up) varies from $\langle\mu\rangle=13.4$ (2015 dataset) to $\langle\mu\rangle=36.1$ (2018 dataset), with a highest $\langle\mu\rangle=37.8$ (2017 dataset) and an average $\langle\mu\rangle=33.7$. Data were collected using a four-jet trigger which, in the HLT, requires four jets each having $|\eta|<2.5$, with $p_{\mathrm{T}}>100 \mathrm{GeV}$ for the 2015-2016 data period and $p_{\mathrm{T}}>120 \mathrm{GeV}$ for the 20172018 data period. Data events used for the validation of the data-driven multijet background were collected using the lowest unprescaled single-lepton triggers; the lowest trigger $p_{\mathrm{T}}$ threshold used for muons is 20 (26) $\mathrm{GeV}$ in 2015 (2016-2018), while for electrons the trigger $p_{\mathrm{T}}$ threshold is 24 (26) GeV in 2015-2017 (2018).

Monte Carlo (MC) simulations are used to model the SUSY signals, as well as to aid in the description of the background processes. In the remainder of this section, the simulation of the signal and of the main background processes contributing to the selected events in data is described. For all the simulated physics processes, the top-quark mass is assumed to be $m_{\text {top }}=172.5 \mathrm{GeV}$ and the Higgs boson mass is taken to be $m_{H}=125 \mathrm{GeV}$. The generation of the simulated event samples includes the effect of multiple $p p$ interactions in the same and neighbouring bunch crossings, as well as the effect of pile-up on the detector response. These interactions were produced using PYTHIA 8.230 [20] with a set of tuned parameters called the A3 tune [21] and the NNPDF2.3 


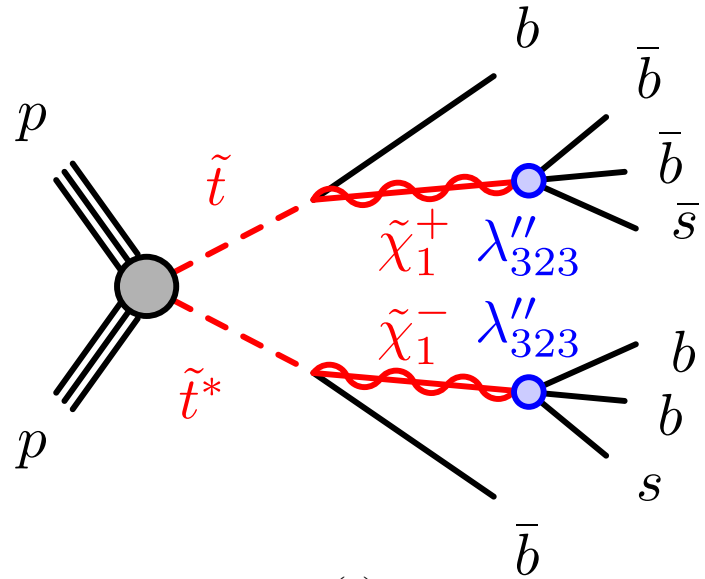

(a)

Fig. 1 Diagrams of the signal processes involving pair production of top squarks $\tilde{t}$ : a with the decay into a $b$-quark and the lightest chargino $\tilde{\chi}_{1}^{+}\left(\tilde{t} \rightarrow b \tilde{\chi}_{1}^{+}\right)$with the subsequent decay of the chargino $\tilde{\chi}_{1}^{+} \rightarrow \bar{b} \bar{b} \bar{s}$

leading-order (LO) [22] parton distribution function (PDF) set.

All generated MC samples were processed through a simulation [23] of the detector geometry and response using either GEANT4 [24] or a fast simulation [25] of the calorimeter response and were then processed by the same reconstruction software used on data. To model the parton shower, hadronisation, and underlying event, the PYTHIA 8 generator was used with the NNPDF2.3 LO PDF set and the A14 [26] set of tunable parameters. The decays of bottom and charm hadrons were modelled using EVTGEN [27]. Simulated MC events are weighted such that the object identification efficiencies, energy scales and energy resolutions match those determined from data control samples [28,29].

MC samples for multijet production were generated using PYTHIA 8.230 with leading-order matrix elements for dijet production and a $p_{\mathrm{T}}$-ordered parton shower. EVTGEN v1.6.0 was used for bottom and charm hadron decays. The renormalisation and factorisation scales were set to the geometric mean of the squared transverse masses of the two outgoing partons, $\sqrt{\left(p_{\mathrm{T}, 1}^{2}+m_{1}^{2}\right)\left(p_{\mathrm{T}, 2}^{2}+m_{2}^{2}\right)}$.

The production of $t \bar{t}$ events (referred to as $t \bar{t}+$ jets) was modelled using the POWHEG-Box v2 [30-33] generator at next-to-leading order (NLO) with the NNPDF3.0 NLO [34] PDF set and with the $h_{\text {damp }}$ parameter ${ }^{2}$ set to $1.5 m_{\text {top }}$ [35]. PYTHIA 8.230 was used for the parton shower and EVTGEN v1.6.0 for bottom and charm hadron decays. The $t \bar{t}+$ jets sample was generated inclusively in the number of

\footnotetext{
2 The $h_{\text {damp }}$ parameter is a resummation damping factor and one of the parameters that controls the matching of POWHEG matrix elements to the parton shower and thus effectively regulates the high- $p_{\mathrm{T}}$ radiation against which the $t \bar{t}$ system recoils.
}

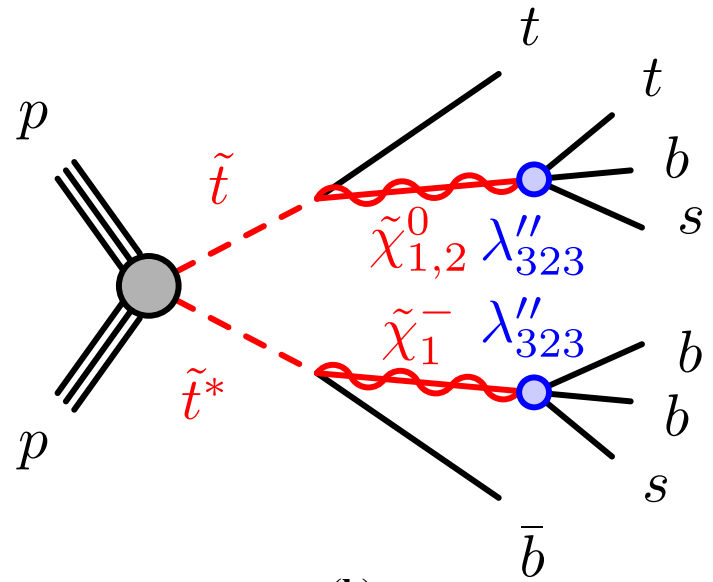

(b)

and charge conjugate (c.c.), and $\mathbf{b}$ the decay into a top quark and the two lightest neutralinos $\tilde{\chi}_{1,2}^{0}$ with the subsequent decay $\tilde{\chi}_{1,2}^{0} \rightarrow t b s$

jets using fast simulation. The MC sample cross-section is corrected to the theory prediction at next-to-next-to-leading order (NNLO) in QCD including resummation of next-tonext-to-leading logarithmic (NNLL) soft gluon terms by means of the Top $++(\mathrm{v} 2.0)$ program [36-42]. The generated events may have jets which do not originate from the decay of the $t \bar{t}$ system. These additional jets are used to categorise the events depending on the flavour of the matching parton. Particle jets are reconstructed from all stable particles generated in the event (excluding muons and neutrinos) using the anti$k_{t}$ algorithm [43] with a radius parameter $R=0.4$ and are required to have $p_{\mathrm{T}}>15 \mathrm{GeV}$ and $|\eta|<2.5$. Events having at least one such particle jet, matched within $\Delta R<0.3$ to a generated $b$-hadron having $p_{\mathrm{T}}>5 \mathrm{GeV}$ and not originating from a top-quark decay, are labelled as $t \bar{t}+\geq 1 b$ events. Similarly, events which are not already categorised as $t \bar{t}+\geq 1 b$, and where at least one particle jet is matched to a $c$-hadron not originating from a $W$ boson decay, are labelled as $t \bar{t}+\geq 1 c$ events. Events labelled as either $t \bar{t}+\geq 1 b$ or $t \bar{t}+\geq 1 c$ are referred to as $t \bar{t}+$ HF events (HF for 'heavy flavour'). The remaining events, including those with no additional jets, are labelled as $t \bar{t}+$ light events (light for 'light flavour').

The $W t$ single-top-quark background was generated at NLO in QCD by POWHEG-BOX v2 with the NNPDF3.0 NLO PDF set. Overlaps between the $t \bar{t}$ and $W t$ final states were removed using the 'diagram removal' scheme [44]. PYTHIA 8.230 was used for the parton shower and EVTGEN v1.6.0 for bottom and charm hadron decays. Samples of single-top events are normalised to the cross-section calculated at NLO in QCD with NNLL soft gluon corrections $[45,46]$. 
The production of $t \bar{t} V$ events was modelled using the MADGRAPH5_aMC@NLO v2.3.3 [47] generator at NLO with the NNPDF3.0 NLO PDF set. PYTHIA 8.210 was used for the parton shower and EVTGEN v1.2.0 for bottom and charm hadron decays.

The production of $t \bar{t} H$ events was modelled using the POWHEG-BOX V2 generator to NLO with the NNPDF3.0 NLO PDF set. PYTHIA 8.230 was used for the parton shower and EVTGENv1.6.0 for bottom and charm hadron decays. The cross-sections are calculated at NLO QCD and NLO electroweak accuracy using the generator MADGRAPH5_ aMC@NLO [48].

Signal events were produced using the MADGRAPH5_aMC@NLOv2.3.3 generator at NLO with the NNPDF2.3 LO PDF, and the fast simulation of the detector response. PYTHIA 8.230 was used for the parton shower and EVTGEN v1.6.0 for bottom and charm hadron decays. Signal cross-section calculations include approximate next-to-nextto-leading-order $\left(\mathrm{NNLO}_{\text {Approx }}\right)$ supersymmetric QCD corrections and the resummation of soft gluon emission at NNLL accuracy [49]. The nominal cross-section and its uncertainty are taken from an envelope of predictions using different PDF sets as well as different factorisation and renormalisation scales. Top-squark masses between $600 \mathrm{GeV}$ and $1 \mathrm{TeV}$ and higgsino masses between $100 \mathrm{GeV}$ and $950 \mathrm{GeV}$ are considered.

\section{Event reconstruction}

Events are required to have a primary vertex reconstructed from at least two tracks with transverse momentum $p_{\mathrm{T}}>$ $500 \mathrm{MeV}$. When several vertices are found in a given bunch crossing, the vertex with the largest summed $p_{\mathrm{T}}^{2}$ of the associated tracks is selected as the primary vertex.

Electrons are reconstructed from energy deposits (clusters) in the electromagnetic calorimeter matched to tracks reconstructed in the ID $[50,51]$ and are required to have $p_{\mathrm{T}}>10 \mathrm{GeV}$ and $|\eta|<2.47$. Candidates in the calorimeter barrel-endcap transition region $(1.37<|\eta|<1.52)$ are excluded. Electron tracks must match the primary vertex of the event: the longitudinal impact parameter $^{3}$ is required to satisfy $\left|z_{0}\right|<0.5 \mathrm{~mm}$, while the transverse impact parameter is required to satisfy $\left|d_{0}\right| / \sigma_{d_{0}}<5$, where $\sigma_{d_{0}}$ represents the uncertainty in the measured $\left|d_{0}\right|$ values. Loose electrons are identified using the 'Medium' identification criterion provided by a likelihood-based discriminant [52]. Tight elec-

\footnotetext{
3 The transverse impact parameter $\left(d_{0}\right)$ is defined as the distance of closest approach in the transverse plane between a track and the beam-line. The longitudinal impact parameter $\left(z_{0}\right)$ corresponds to the $z$-coordinate difference between the point along the track at which the transverse impact parameter is defined and the primary vertex.
}

trons are required to pass the 'TightLH' selection [52] and the 'Gradient' isolation criteria [52] and $p_{\mathrm{T}}>27 \mathrm{GeV}$.

Muons are reconstructed by matching either track segments or full tracks in the MS to tracks in the ID [53]. Combined tracks are then re-fitted using information from both detector systems. Muon tracks must match the primary vertex of the event: the longitudinal impact parameter is required to satisfy $\left|z_{0}\right|<0.5 \mathrm{~mm}$, while the transverse impact parameter is required to satisfy $\left|d_{0}\right| / \sigma_{d_{0}}<3$. Loose muons are those that pass the 'Loose' muon selection [53] and have $p_{\mathrm{T}}>10 \mathrm{GeV}$ and $|\eta|<2.5$, and Tight muons are those that pass the 'Medium' muon selection [53], satisfy the 'FixedCutTightTrackOnly' isolation criterion [53], and have $p_{\mathrm{T}}>27 \mathrm{GeV}$.

Jets are reconstructed from three-dimensional topological energy clusters [54] in the calorimeter using the anti- $k_{t}$ jet algorithm [43] with a radius parameter of 0.4. Reconstructed jets are then corrected to the particle level by the application of a jet energy scale calibration that is derived from simulation and by in situ corrections obtained from $13 \mathrm{TeV}$ data [55]. Jets used in this analysis are required to have $p_{\mathrm{T}}>25 \mathrm{GeV}$ and $|\eta|<2.5$ after calibration.

To avoid selecting jets from pile-up, low- $p_{\mathrm{T}}\left(p_{\mathrm{T}}<\right.$ $120 \mathrm{GeV})$ jets in the central $(|\eta|<2.5)$ region of the detector are required to satisfy the jet-vertex tagger [56] configured such that it has an efficiency of approximately $92 \%$ to identify jets from a primary vertex. This requirement is applied to both data and simulation. Quality criteria are imposed to identify jets arising from non-collision sources or detector noise (using the BadLoose operating point [57]), and any event containing at least one such jet is removed. This removal produces a negligible loss of efficiency for signal events.

The $b$-jets are identified via a $b$-tagging algorithm that uses multivariate techniques to combine information from the impact parameters of displaced tracks as well as topological properties of secondary and tertiary decay vertices reconstructed within the jet. This analysis uses the MV2c10 tagger [58], trained on a hybrid sample of simulated $t \bar{t}$ and $Z^{\prime}$ events statistically enriched at high- $p_{\mathrm{T}}$ in order to discriminate $b$-jets from a background consisting of light- (93\%) and $c$-labelled (7\%) jets [29]. A weight is calculated corresponding to the probable presence of a $b$-quark or a $c$-quark, and jets are confirmed $b$-tagged if they satisfy a minimum requirement on the MV2c10 $b$-tagging weight corresponding to an average efficiency in $t \bar{t}$ events of $60 \%$ for $b$-jets, $4 \%$ for $c$-jets and a rejection factor of approximately 1200 for light-flavour jets across the jet $p_{\mathrm{T}}$ range.

An overlap removal procedure is carried out to resolve ambiguities between jets and lepton candidates. To prevent treating electron energy deposits as jets, the closest jet within $\Delta R_{y}=\sqrt{(\Delta y)^{2}+(\Delta \phi)^{2}}=0.2$ of a selected electron is 
removed. ${ }^{4}$ If the nearest jet surviving that selection is within $\Delta R_{y}=0.4$ of the electron, the electron is discarded. To reduce the background from heavy-flavour decays inside jets, muons are removed if they are separated from the nearest jet by $\Delta R_{y}<0.4$. However, if that jet has fewer than three associated tracks, the muon is kept and the jet is removed instead.

\section{Analysis strategy}

Events selected for further analysis are required to have at least five jets, of which at least two must be $b$-tagged. The four highest- $p_{\mathrm{T}}$ jets are required to be on the trigger efficiency plateau, namely to have $p_{\mathrm{T}}>120 \mathrm{GeV}$ or $p_{\mathrm{T}}>140 \mathrm{GeV}$, depending on the jet- $p_{\mathrm{T}}$ trigger requirement in 2015-2016 or 2017-2018, and have $|\eta|<2.5$. All other jets present in the event are required to have $p_{\mathrm{T}}>25 \mathrm{GeV}$ and $|\eta|<2.5$. A lepton veto is applied: events that contain loose muons or electrons with $p_{\mathrm{T}}>10 \mathrm{GeV}$, whether isolated or nonisolated, are discarded.

After the selections described above, the largest background contribution to the measurement is from non-resonant multijet production from light-quark and gluonic final states. The next largest is from $t \bar{t}+$ jets production. Other small background contributions originate from the production of a single top quark and from the production of a $t \bar{t}$ pair in association with either a vector boson or a Higgs boson. The estimation of the multijet background using a data-driven method and the validation of this estimate without significant bias from potential signal contamination are the main challenges for this analysis.

To probe top-squark pair production and estimate the contribution of signal top squarks in data, a model-dependent fit of the yield of events with jet multiplicity $N_{\mathrm{j}}=6,7,8$ and $\geq$ 9 and $b$-tagged jet multiplicity $N_{\mathrm{b}}=4$ and $\geq 5$ is performed. These $\left(N_{\mathrm{j}}, N_{\mathrm{b}}\right)$ regions are indicated as $\mathrm{SR}_{\tilde{t}}$ in Table 1 . The signal contribution predicted for different values of $m_{\tilde{f}}$ and $m_{\tilde{\chi}_{1,2}^{0}, \tilde{\chi}_{1}^{ \pm}}$is considered in all bins and is scaled by one common signal-strength parameter $\left(\mu_{\tilde{t} \tilde{t}^{*}}\right)$. For the model considered here, the product of acceptance and reconstruction efficiency $(\mathrm{A} \times \epsilon)$ is of order $\sim 5 \times 10^{-2}$ for $N_{\mathrm{j}} \geq 9$ and $N_{\mathrm{b}} \geq$ 5. Figure 2 shows the number of signal events obtained from the model as a function of $N_{\mathrm{j}}$ and $N_{\mathrm{b}}$ compared to the estimated backgrounds. Their evaluation is described in Sect. 6. The signal yields are concentrated at high jet and $b$-tagged jet multiplicity, while the backgrounds are concentrated at low $b$-tagged jet multiplicity. To validate the background estimates, intervals with $N_{\mathrm{j}}=6,7,8$ and $\geq 9$, and $N_{\mathrm{b}}=3$ and 4 , subsequently referred to as VR-MJ, are used. In these, a

\footnotetext{
$\overline{4}$ The rapidity is defined as $y=\frac{1}{2} \ln \frac{E+p_{\mathrm{Z}}}{E-p_{\mathrm{z}}}$ where $E$ is the energy and $p_{\mathrm{Z}}$ is the longitudinal component of the momentum along the beam-line.
}

region-dependent selection is applied, based on a maximum accepted value of the centrality mass $\left(C_{\text {mass }}\right)$, defined as:

$C_{\text {mass }}=\frac{H_{\mathrm{T}}}{\sqrt{\left(\sum_{i=1}^{N_{\mathrm{j}}} E_{i}\right)^{2}-\left(\sum_{i=1}^{N_{\mathrm{j}}} \mathbf{p}_{i}\right)^{2}}}$,

i.e. the ratio of the scalar sum of all jet $p_{\mathrm{T}}$ in the event $\left(H_{\mathrm{T}}\right)$ to the invariant mass of the set of observed jets. The signalto-background ratio decreases monotonically with decreasing $C_{\text {mass }}$ for all $N_{\mathrm{j}}$ and $N_{\mathrm{b}}$ values. The value of the maximum value of $C_{\text {mass }}\left(C_{\operatorname{mass}}^{\max }\right)$ is chosen such that the signal-tobackground ratio is less than $5 \%$. Values of the $C_{\operatorname{mass}}^{\max }$ limits used are listed in Table 1.

A separate, model-independent test is used to search for, and to set generic exclusion limits on, potential contributions from a hypothetical BSM signal by comparing the observed number of events with background predictions in two dedicated signal regions, one with $N_{\mathrm{j}} \geq 9$ and $N_{\mathrm{b}} \geq 5$ and the other with $N_{\mathrm{j}} \geq 8$ and $N_{\mathrm{b}} \geq 5$ (labelled SR discovery in Table 1), that were not explored in previous searches at the LHC.

\section{Multijet background estimation}

The predominant multijet background is estimated via a datadriven method, subsequently referred to as the tag-rate function method for multijet events $\left(\mathrm{TRF}_{\mathrm{MJ}}\right)[59,60]$. The aim is to extrapolate the $b$-tag multiplicity distributions from $N_{\mathrm{j}}$ $=5$, where the signal contamination for models not already excluded by other LHC searches is negligible, to larger $N_{\mathrm{j}}$ values. The $\mathrm{TRF}_{\mathrm{MJ}}$ method uses a tag-rate function to quantify the experimental probability of $b$-tagging an additional jet in samples of events with at least two, or at least three, $b$ tagged jets. This per-jet probability is then used to estimate the shape of the multijet $b$-tag multiplicity distribution for each $N_{\mathrm{j}}$ value.

Events that satisfy the selection criteria described in Sect. 5 and that have exactly five jets, of which at least two are $b$-tagged, are used to determine the $b$-tagging probability. The data are first corrected by subtracting the expected nonmultijet background found in simulation, approximately $5 \%$ of the total. After excluding the two jets in each event with the highest $b$-tagging weight, the probability that each remaining jet is $b$-tagged, denoted $\varepsilon_{2}$, is calculated for this jet. A similar procedure is used to calculate the probability $\varepsilon_{3}$ of additional $b$-tagged jets in events with at least three $b$-tagged jets. These $\varepsilon$ probabilities are parameterised as a function of both the $p_{\mathrm{T}}$ of the remaining jet divided by $H_{\mathrm{T}}$, and the minimum $\Delta R$ between that jet and the two (for $\varepsilon_{2}$ ) or three (for $\varepsilon_{3}$ ) jets with the largest $b$-tagging weight in the event $\left(\Delta R_{\min }\right)$. This choice of variables for the parameterisation is made to minimise the residual differences (non-closure) between the 
Table 1 The strategy of the analysis. For the model-dependent fit, the signal regions $\left(\mathrm{SR}_{\tilde{t}}\right)$ consist of events with $N_{\mathrm{j}}=6,7,8$ and $\geq 9$ jets and $N_{\mathrm{b}}=4$ and $\geq 5$. These are used independently in the final fit. For the model-independent fit, two dedicated signal regions $\left(\mathrm{SR}_{\text {discovery }}\right)$, with $\left(N_{\mathrm{j}} \geq 9, N_{\mathrm{b}} \geq 5\right)$ and $\left(N_{\mathrm{j}} \geq 8, N_{\mathrm{b}} \geq 5\right)$, are used. The validation regions (VR-MJ), which are based on a maximum value of the centrality mass, $C_{\operatorname{mass}}^{\max }$, introduced for the description of the VRs in Sect. 5, are also indicated

\begin{tabular}{lllll}
\hline Analysis regions & \multicolumn{2}{l}{$N_{\mathrm{b}}$} & & \\
\cline { 2 - 4 } & & 3 & 4 \\
\hline
\end{tabular}

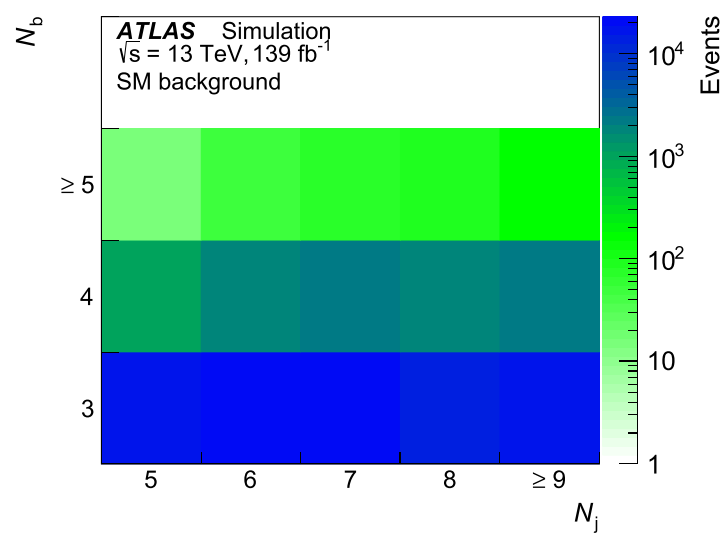

(a)

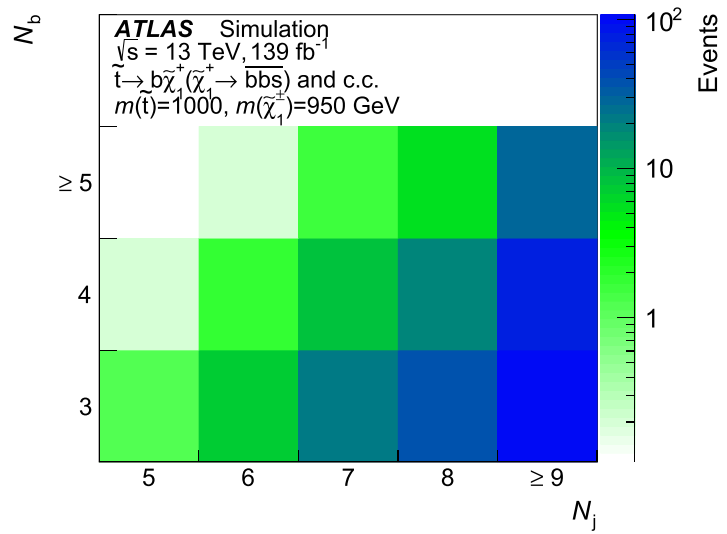

(b)

Fig. 2 Predicted numbers of events as a function of jet multiplicity, $N_{\mathrm{j}}$, and $b$-tagged jet multiplicity, $N_{\mathrm{b}}$, for a SM background (multijet and top-quark production) and $\mathbf{b}$ top-squark pair production in the $\tilde{t} \rightarrow \bar{b} \tilde{\chi}_{1}^{+}\left(\tilde{\chi}_{1}^{+} \rightarrow \bar{b} \bar{b} \bar{s}\right.$ ) (and c.c.) channel, for $m_{\tilde{t}}=1000 \mathrm{GeV}$ and $m_{\tilde{\chi}_{1}^{ \pm}}=950 \mathrm{GeV}$

$\mathrm{TRF}_{\mathrm{MJ}}$ prediction and the number of events obtained when selecting $b$-jets directly in the most sensitive signal regions in the multijet events simulated by MC. The dependence of $\varepsilon_{2}$ and $\varepsilon_{3}$ on both $p_{\mathrm{T}} / H_{\mathrm{T}}$ and $\Delta R_{\min }$ is shown in Fig. 3. The rapid variation with $\Delta R_{\min }$ is consistent with the dependence expected from multi- $b$-jet production due to gluon-splitting. The $p_{\mathrm{T}} / H_{\mathrm{T}}$ dependence, more visible at small $\Delta R_{\text {min }}$, reflects the variation of the $b$-tagging efficiency with jet $p_{\mathrm{T}}$.

Following the methods of Ref. [61], in the second step of the $\mathrm{TRF}_{\mathrm{MJ}}$ method the expected number of events with each different number of $b$-tagged jets is estimated for each $N_{\mathrm{j}}$ value by weighting all events with $N_{\mathrm{b}} \geq 2$ by the event probability of having $N_{\mathrm{b}}=2,3,4$ and $\geq 5$, respectively. Upon subtracting the non-multijet background contribution [59], the event probabilities are estimated using both $\varepsilon_{2}$ and $\varepsilon_{3}$, after first excluding the two jets with the highest $b$-tagging weight. For $N_{\mathrm{b}}=2$ the event probabilities are estimated directly from $\varepsilon_{2}$, treating the tagging probability for each jet as independent. For $N_{\mathrm{b}}=3,4$ and $\geq 5$, a two-step procedure is employed. First, a 'pseudodata sample' with $N_{\mathrm{b}} \geq 3$ is emulated, using $\varepsilon_{2}$ in events with $N_{\mathrm{b}} \geq 2$. The additional emulated $b$-tagged jet is chosen randomly from the remaining $N_{\mathrm{j}}-2$ jets by using their probability-dependent $b$-tagging weights [60]. This emulated sample is then used to estimate the event probabilities, this time relying on $\varepsilon_{3}$. The probability of finding $N_{\mathrm{b}}=4$ and $N_{\mathrm{b}} \geq 5$ is estimated using the emulated $N_{\mathrm{b}} \geq 3$ sample via $\varepsilon_{3}$. Due to too few events in the control sample from which the $\varepsilon_{2}$ and $\varepsilon_{3}$ values are extracted, it is not possible to estimate the probability of $b$-tagging an additional jet in a sample of events with at least four $b$-tagged jets.

\subsection{Validation of $\mathrm{TRF}_{\mathrm{MJ}}$ method}

The TRF $F_{M J}$ method is validated using two different comparisons with data: in the VR-MJ regions defined in Sect. 5, and in a separate set of $Z+$ jets-enriched events. Figure 4 shows 


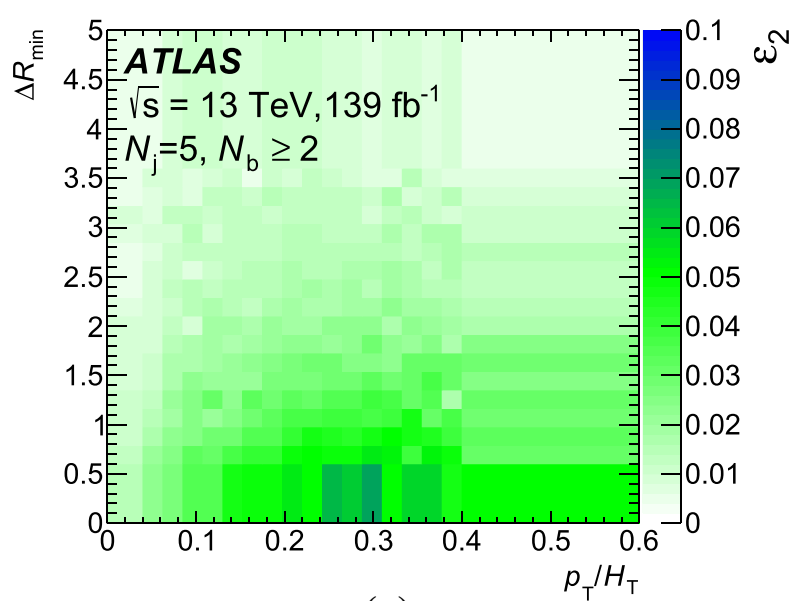

(a)

Fig. 3 Two-dimensional distributions of the probability $\mathbf{a} \varepsilon_{2}$ or $\mathbf{b} \varepsilon_{3}$ of $b$-tagging an additional jet in a sample of events with $\mathbf{a}$ at least two or $\mathbf{b}$ at least three $b$-tagged jets as a function of the ratio of jet transverse momentum to $H_{\mathrm{T}}, p_{\mathrm{T}} / H_{\mathrm{T}}$, and the minimum $\Delta R$ between the jet and

a comparison between measured and estimated event rates in VR-MJ. The data and predictions are in agreement within systematic uncertainties (described in Sect. 7).

An independent test of the method is performed in $Z+$ jetsenriched events, referred as 'VR-ZJ', where additional jets are produced by radiation and where $b \bar{b}$ pairs arise from gluon splitting. In order to select events where a $Z$ boson decays into pairs of electrons or muons, events are required to pass a single-lepton trigger. Two opposite-sign, same-flavour, tight electrons or muons are required to each have $p_{\mathrm{T}}>27 \mathrm{GeV}$ and a pair mass larger than $60 \mathrm{GeV}$. Events are required to have at least five jets with $p_{\mathrm{T}}>25 \mathrm{GeV}$ and $|\eta|<2.5$, of which at least two must be $b$-tagged. The tagging probabilities $\varepsilon_{2}$ and $\varepsilon_{3}$ are derived from five-jet VR-ZJ events and used to predict the number of events with $N_{\mathrm{j}}=6,7,8, \geq 9$ and $N_{\mathrm{b}}=4, \geq 5$. As shown in Fig. 5, this statistically limited test further validates the $\mathrm{TRF}_{\mathrm{MJ}}$ method.

\section{Systematic uncertainties}

Several sources of systematic uncertainty are considered that can affect the overall normalisation of signal and background samples and their relative contribution for different values of $N_{\mathrm{j}}$ and $N_{\mathrm{b}}$. In estimating the dominant multijet background from the data, systematic uncertainties arise from the assumptions made in obtaining the $\mathrm{TRF}_{\mathrm{MJ}}$ background estimates. Uncertainties related to the theoretical modelling and due to the description of the detector response in simulated events are relevant only for the signal and background MC samples.

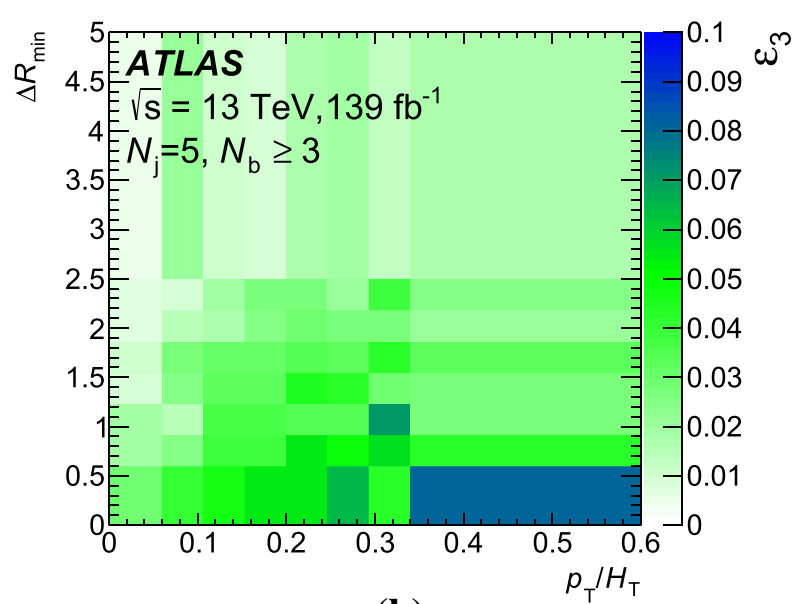

(b)

the a two or $\mathbf{b}$ three $b$-tagged jets with the highest $b$-tagging weight in the event, $\Delta R_{\min }$. The choice of binning is made so as to avoid empty bins

The main assumption of the $\mathrm{TRF}_{\mathrm{MJ}}$ method is that it is possible to define per-jet $b$-tagging probabilities $\left(\varepsilon_{2}\right.$ and $\left.\varepsilon_{3}\right)$ in events with at least two or at least three $b$-tagged jets and, in particular, that the variables used for the parameterisation are sensitive to the heavy-flavour composition of the jet sample. A second assumption is that the per-jet probabilities are independent of the jet multiplicity and, therefore, may be derived in a specific region, namely that with exactly five jets, and applied to regions with $N_{\mathrm{j}}=6,7,8$ and $\geq 9$ jets. The validity of these assumptions is verified using MC simulations. The $\mathrm{TRF}_{\mathrm{MJ}}$ method is applied to PYTHIA $8 \mathrm{MC}$ dijet events, and the larger of (a) the residual non-closure and (b) the statistical uncertainty in the number of events with a given $b$-tagged jet multiplicity, is symmetrised and taken to be the systematic uncertainty associated with the method. Table 2 shows the final $\mathrm{TRF}_{\mathrm{MJ}}$ systematic uncertainty in the multijet background estimation in each $\left(N_{\mathrm{j}}, N_{\mathrm{b}}\right)$ region. For $N_{\mathrm{b}}=$ 4 the $\mathrm{TRF}_{\mathrm{MJ}}$ uncertainties are dominated by the non-closure component, while for $N_{\mathrm{b}} \geq 5$, the statistical component dominates. The $\mathrm{TRF}_{\mathrm{MJ}}$ uncertainties are the source of the largest systematic uncertainty for the analysis.

The second largest contribution to the total systematic uncertainty arises from the modelling of the $t \bar{t}+$ jets background. The diagrams that contribute to $t \bar{t}+\geq 1 b, t \bar{t}+\geq 1 c$, and $t \bar{t}+$ light production are different, and the associated uncertainties may affect these processes differently in different regions. As a result, all uncertainties in $t \bar{t}+$ jets background modelling, except the uncertainty in the inclusive cross-section, are considered to be uncorrelated among $t \bar{t}+\geq 1 b, t \bar{t}+\geq 1 c$, and $t \bar{t}+$ light. 
Fig. 4 Comparison between data and the predicted number of events with $N_{\mathrm{j}}=6,7,8$ and $\geq 9$ and $N_{\mathrm{b}}=3$ and 4 in the VR-MJ validation regions, which are based on a maximum value of the centrality mass, $C_{\text {mass. }}$. The bottom panel displays the ratios of data to the are statistical only. The systematic uncertainties listed in Sect. 7 are represented by the blue hatched area total prediction, uncertainty bars

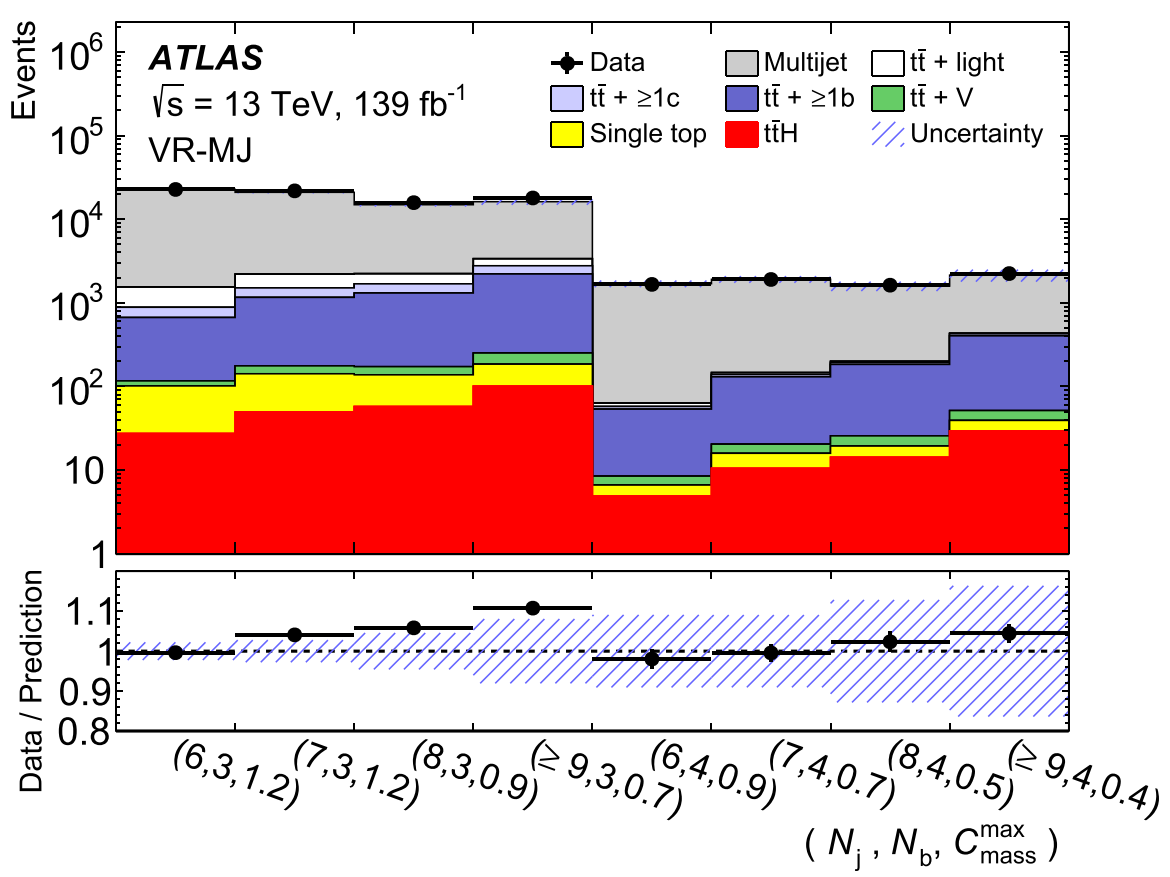

Fig. 5 Comparison between data and the number of events with $N_{\mathrm{j}}=6,7,8$ and $\geq 9$ and $N_{\mathrm{b}}=4$ and $\geq 5$ predicted by the $\mathrm{TRF}_{\mathrm{MJ}}$ method (grey histogram) in the VR-ZJ region, defined by the requirement of two isolated leptons with invariant mass larger than $60 \mathrm{GeV}$. The bottom panel displays the ratios of data to the $\mathrm{TRF}_{\mathrm{MJ}}$ prediction, uncertainty bars are statistical only. Systematic uncertainties in the $\mathrm{TRF}_{\mathrm{MJ}}$ prediction are represented by the blue hatched area

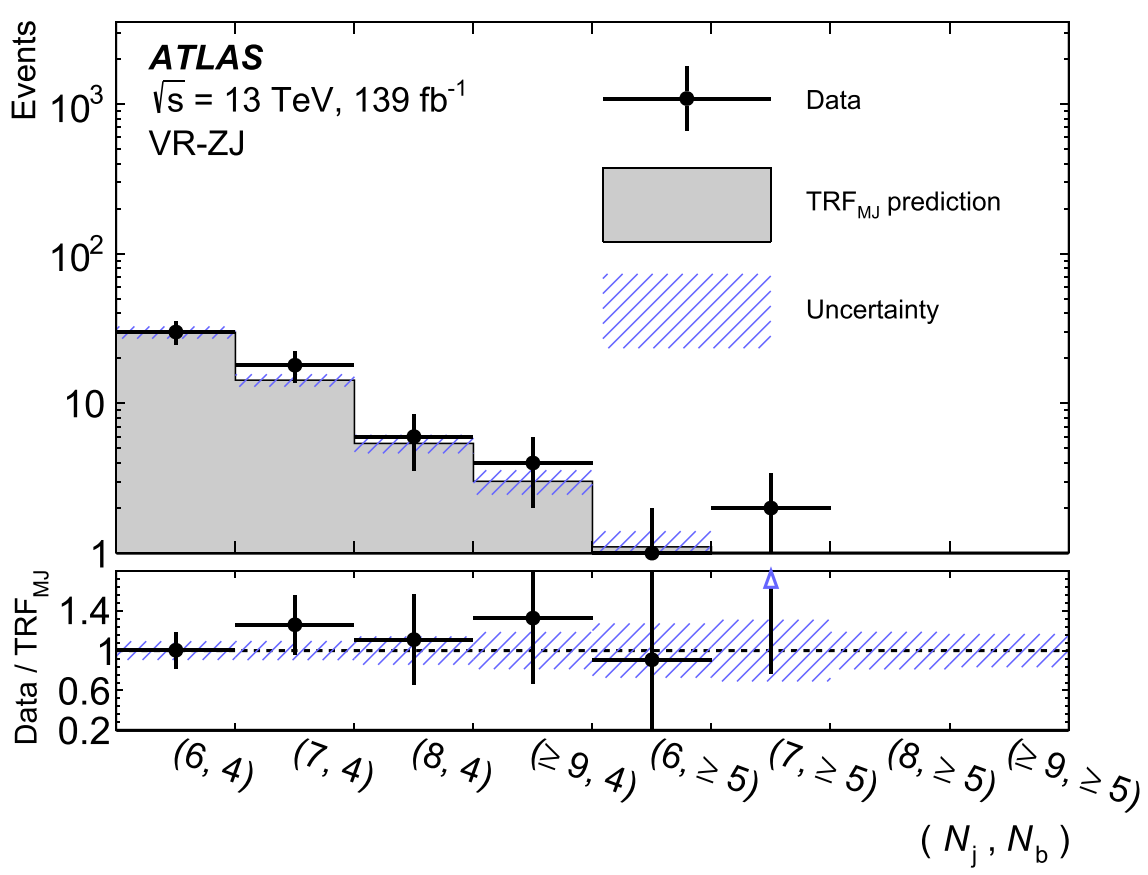

The uncertainty in the inclusive $t \bar{t}$ NNLO+NNLL production cross-section is taken to be $\pm 6 \%$ [42]. This uncertainty includes effects from varying the factorisation and renormalisation scales, the PDF, $\alpha_{\mathrm{S}}$, and the top-quark mass. The normalisations of the $t \bar{t}+\geq 1 c$ and $t \bar{t}+\geq 1 b$ yields are taken from their fractional contribution to the nominal $t \bar{t}+$ jets sample as generated using the POWHEG-Box program. In addi- tion to the uncertainty in the inclusive $t \bar{t}$ cross-section, an additional uncertainty of $50 \%$, based on the measurement of the $t \bar{t}+\geq 1 b$ and $t \bar{t}+\geq 1 c$ normalisation factors reported in Ref. [62], is assigned to the $t \bar{t}+\geq 1 c$ and $t \bar{t}+\geq 1 b$ production cross-sections.

The impact of the parton shower and hadronisation model uncertainties on the $t \bar{t}+$ jets, $t \bar{t} H$ and $W t$ single-top-quark 
Table 2 Systematic uncertainties in the data-driven estimation of the multijet background using the $\mathrm{TRF}_{\mathrm{MJ}}$ method. The uncertainties are assessed using PYTHIA 8 MC dijet events for each value of jet multiplicity $\left(N_{\mathrm{j}}\right)$ and $b$-tagged jet multiplicity $\left(N_{\mathrm{b}}\right)$ used in the final fit

\begin{tabular}{llll}
\hline TRF & & \multicolumn{2}{l}{$N_{\mathrm{b}}$} \\
\cline { 3 - 4 } & & 4 & $\geq 5$ \\
\hline$N_{\mathrm{j}}$ & 6 & $9 \%$ & $27 \%$ \\
& 7 & $9 \%$ & $30 \%$ \\
& 8 & $13 \%$ & $18 \%$ \\
& $\geq 9$ & $16 \%$ & $14 \%$ \\
\hline
\end{tabular}

yields is evaluated by comparing the sample from the nominal generator set-up with a sample produced with the NLO POWHEG-BOX v2 generator using the NNPDF3.0 NLO PDF set. The latter events are interfaced with HERwIG 7.04 $[63,64]$, using the H7UE set of tuned parameters [64] and the MMHT2014LO PDF set [65], and processed using fast simulation of the detector response. The difference between the two predictions of the $t \bar{t}+\geq 1 b$ event yield ranges from $20 \%(33 \%)$ for $N_{\mathrm{j}}=6$ and $N_{\mathrm{b}}=4(\geq 5)$ to $46 \%(60 \%)$ in the region with $N_{j} \geq 9$ and $N_{\mathrm{b}}=4(\geq 5)$.

To assess the uncertainty due to the choice of matching scheme, the POWHEG-BOX sample is compared with a sample produced by MADGRAPH5_aMC@NLO and PYTHIA 8 . For the calculation of the hard scattering, MADGRAPH5_ aMC@NLO v2.6.0 with the NNPDF3.0 NLO PDF set is used. The events are processed with PYTHIA 8.230, using the A14 set of tuned parameters and the NNPDF2.3 LO PDF set, and the fast simulation of the detector response. The uncertainty, which is obtained from the difference in yield between the two models and is symmetrised, affects both the normalisation and the $N_{\mathrm{j}}$ - and $N_{\mathrm{b}}$-dependence of background rates. It is largest for large values of the jet and $b$-tagged jet multiplicities. For $t \bar{t}+\geq 1 b$, it reaches $25 \%$ for $N_{\mathrm{j}}=8, \geq 9$ and $N_{\mathrm{b}}=4$, and $41 \%(32 \%)$ for $N_{\mathrm{j}}=8(\geq 9)$ and $N_{\mathrm{b}} \geq 5$.

The effect of renormalisation and factorisation scale uncertainties and PDF uncertainties is evaluated for $t \bar{t} H$ and $t \bar{t} V$ events. For the former, the scales are varied simultaneously by common factors of 2.0 and 0.5 . For the latter, the envelope of the 100 variations for NNPDF3.0 NLO [34] are taken into account. An uncertainty of $\pm 5 \%$ is assigned to the total cross-section for single-top production $[45,66,67]$. For both the $t \bar{t} H$ and single-top events, additional uncertainties due to initial- and final-state radiation and the choice of generator are evaluated in a manner similar to that used for $t \bar{t}+$ jets. The uncertainty in the amount of interference between $W t$ and $t \bar{t}$ production at NLO is assessed by comparing samples using the default 'diagram removal' scheme with those using an alternative 'diagram subtraction' scheme [44]. All modelling uncertainties from non- $t \bar{t}+$ jets simulated backgrounds are, after investigation, found to be negligible.
The uncertainties assigned to the expected signal yield for the SUSY benchmark processes considered include the experimental uncertainties related to the luminosity and to the detector modelling, which are dominated by the modelling of the jet energy scale and the $b$-tagging efficiencies. For example, for the $\tilde{t} \rightarrow b \tilde{\chi}_{1}^{+}\left(\tilde{\chi}_{1}^{+} \rightarrow \bar{b} \bar{b} \bar{s}\right.$ and c.c.) signal model, the $b$-tagging uncertainties in the region $N_{j} \geq 9$ and $N_{\mathrm{b}}=4$ are approximatively $10 \%$, and the jet-related uncertainties of the signal yields are in the range of $3-5 \%$. The uncertainties in the signal yields related to the modelling of additional jet radiation are studied by varying the factorisation, renormalisation, and jet-matching scales as well as the parton-shower tune in the simulation. The corresponding uncertainties are small for most of the signal parameter space and are largest for small top-squark masses, where they reach $7 \%$. The uncertainty in the signal cross-section ranges between $8 \%$ and $11 \%$ for a top-squark mass in the range 600-1000 GeV.

\section{Results}

The events are allocated to $\left(N_{\mathrm{j}}, N_{\mathrm{b}}\right)$ regions with different signal-to-background ratios in order to constrain systematic uncertainties and to improve the separation of signal and background. Then, in each region, the total signal and background yields, shown in Tables 3 and 4, are used in combination as the input for the statistical analysis to extract the final results.

Hypothesis testing is performed using a modified frequentist method as implemented in ROOSTATS [68] and is based on a profile likelihood which takes into account the systematic uncertainties as nuisance parameters. This procedure minimises the impact of systematic uncertainties on the search sensitivity by taking advantage of the highly populated, background-dominated $\left(N_{\mathrm{j}}, N_{\mathrm{b}}\right)$ regions included in the likelihood fit. The signal-strength parameter, $\mu_{\tilde{t} \tilde{t}^{*}}$, defined for positive values and corresponding to the signal normalisation, is unconstrained in the profile-likelihood fit. The normalisation of each component of the background and $\mu_{\tilde{t} \tilde{t}^{*}}$ are determined simultaneously from the fit to the data.

Individual sources of systematic uncertainty are taken as uncorrelated. Contributions from $t \bar{t}+\geq 1 b, t \bar{t}+\geq 1 c, t \bar{t}$ + light, $t \bar{t}+V, t \bar{t} H$ and single-top-quark backgrounds are constrained by the uncertainties of the respective theoretical calculations, the uncertainty in the luminosity (described in Sect. 3), and experimental data. The $\mathrm{TRF}_{\mathrm{MJ}}$ uncertainty is taken as uncorrelated across regions because of its large statistical component. In all cases, the profile-likelihoodratio test is used to establish $95 \%$ confidence intervals using the $\mathrm{CL}_{\mathrm{s}}$ [69] prescription. The likelihood is configured differently for the model-independent and model-dependent hypothesis tests. 
Table 3 Event yields from background predictions and data in the regions with $N_{\mathrm{j}}=6,7,8$ or $\geq 9$ and $N_{\mathrm{b}}=4$. The quoted uncertainties are the sum in quadrature of the statistical and systematic uncertainties in the yields for all samples. The individual background uncertainties can be larger than the total uncertainty due to correlations between parameters

\begin{tabular}{lllll}
\hline Process & $\left(N_{\mathrm{j}}, N_{\mathrm{b}}\right)$ & & & $(8,4)$ \\
\cline { 2 - 5 } & $(6,4)$ & $(7,4)$ & $1510 \pm 210$ & $(\geq 9,4)$ \\
\hline Multijet & $1760 \pm 170$ & $1920 \pm 180$ & $6 \pm 4$ & $1870 \pm 350$ \\
$t \bar{t}+$ light & $6 \pm 4$ & $8.0 \pm 3.4$ & $11 \pm 6$ & $8 \pm 7$ \\
$t \bar{t}+\geq 1 c$ & $4.1 \pm 2.9$ & $8 \pm 5$ & $160 \pm 100$ & $22 \pm 17$ \\
$t \bar{t}+\geq 1 b$ & $45 \pm 26$ & $110 \pm 70$ & $0.30 \pm 0.10$ & $350 \pm 260$ \\
$t \bar{t}+W$ & $0.055 \pm 0.032$ & $0.26 \pm 0.07$ & $6.0 \pm 1.5$ & $1.34 \pm 0.28$ \\
$t \bar{t}+Z$ & $1.8 \pm 0.4$ & $4.3 \pm 1.0$ & $5.1 \pm 3.1$ & $10.9 \pm 2.3$ \\
$W t$ & $1.7 \pm 2.0$ & $5 \pm 5$ & $14.2 \pm 2.4$ & $29 \pm 8$ \\
$t \bar{t} H$ & $4.9 \pm 0.9$ & $10.5 \pm 1.7$ & $1710 \pm 220$ & $2300 \pm 400$ \\
\hline Total background & $1820 \pm 170$ & $2060 \pm 190$ & 1624 & 2237 \\
Data & 1660 & 1901 & & \\
\hline
\end{tabular}

Table 4 Event yields from background predictions and data in the regions with $N_{\mathrm{j}}=6,7,8$ or $\geq 9$ and $N_{\mathrm{b}} \geq 5$. The quoted uncertainties are the sum in quadrature of the statistical and systematic uncertainties in the yields for all samples. The individual background uncertainties can be larger than the total uncertainty due to correlations between parameters

\begin{tabular}{lllll}
\hline Process & $\left(N_{\mathrm{j}}, N_{\mathrm{b}}\right)$ & & & $(8, \geq 5)$ \\
\cline { 2 - 5 } & $(6, \geq 5)$ & $(7, \geq 5)$ & $74 \pm 14$ & $(\geq 9, \geq 5)$ \\
\hline Multijet & $49 \pm 13$ & $75 \pm 23$ & $<0.01$ & $123 \pm 20$ \\
$t \bar{t}+$ light & $<0.01$ & $0.3 \pm 0.6$ & $0.3 \pm 0.4$ & $0.00 \pm 0.04$ \\
$t \bar{t}+\geq 1 c$ & $<0.01$ & $0.016 \pm 0.029$ & $7 \pm 6$ & $0.26 \pm 0.31$ \\
$t \bar{t}+\geq 1 b$ & $1.2 \pm 0.9$ & $3.9 \pm 2.7$ & $0.021 \pm 0.025$ & $28 \pm 25$ \\
$t \bar{t}+W$ & $<0.01$ & $0.005 \pm 0.007$ & $0.7 \pm 0.4$ & $0.090 \pm 0.035$ \\
$t \bar{t}+Z$ & $0.05 \pm 0.05$ & $0.22 \pm 0.12$ & $0.00 \pm 0.13$ & $0.7 \pm 0.7$ \\
$W t$ & $<0.01$ & $<0.01$ & $0.82 \pm 0.21$ & $0.9 \pm 1.2$ \\
$t \bar{t} H$ & $0.12 \pm 0.05$ & $0.49 \pm 0.13$ & $84 \pm 15$ & $2.9 \pm 1.5$ \\
\hline Total background & $50 \pm 13$ & $80 \pm 23$ & 80 & $156 \pm 27$ \\
Data & 35 & 75 & & 179 \\
\hline
\end{tabular}

For the model-independent test, a profile-likelihood fit is performed independently in the two $\mathrm{SR}_{\text {discovery }}$ regions with $\left(N_{\mathrm{j}} \geq 8, N_{\mathrm{b}} \geq 5\right)$ and $\left(N_{\mathrm{j}} \geq 9, N_{\mathrm{b}} \geq 5\right)$. This test is used to search for, and to compute generic exclusion limits on, the potential contribution from a hypothetical BSM signal in the given $\mathrm{SR}_{\text {discovery }}$ regions.

For the model-dependent test, assuming a specific topsquark model with variable mass values, tests of the signalplus-background hypothesis, i.e. $\mu_{\tilde{t} \tilde{t}^{*}}=1$, are formed for a

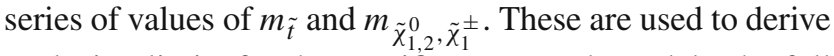
exclusion limits for the specific top-squark model. The full set of regions, $N_{\mathrm{j}}=6,7,8$ and $\geq 9$ and $N_{\mathrm{b}}=4$ and $\geq 5$, is employed in the likelihood. The expected signal contribution, as predicted by the given model, is considered in all regions and is scaled by $\mu_{\tilde{t} \tilde{t}^{*}}$.
Figure 6 shows the observed numbers of data events compared with the fitted background model. The likelihood fit is configured using the model-dependent set-up where all bins are input to the fit, and $\mu_{\tilde{t} \tilde{t}^{*}}$ is set to zero. This configuration is also referred to as the background-only fit and includes no free-floating parameters, only nuisance parameters with Gaussian constraints. An example signal model is also shown in the figure to illustrate the separation between the signal and the background.

\subsection{Model-independent interpretation}

The model-independent results are calculated from the observed number of events and the background predictions in the two $\mathrm{SR}_{\text {discovery }}$ regions. The observed number of events and the backgrounds obtained from the fits are shown for both $\mathrm{SR}_{\text {discovery }}$ regions in Table 5 . 
Fig. 6 Expected background and observed number of events in different jet and $b$-tag multiplicity bins. The background is estimated by including all bins in a background-only fit and is plotted separately for each contribution. An example signal yield for $\tilde{t} \rightarrow \bar{b} \tilde{\chi}_{1}^{+}\left(\tilde{\chi}_{1}^{+} \rightarrow \bar{b} \bar{b} \bar{s}\right.$ and c.c.) production with $m_{\tilde{t}}=$ $600 \mathrm{GeV}$ and $m_{\tilde{\chi}_{1}^{ \pm}}=550 \mathrm{GeV}$ is overlaid. The bottom panel displays the ratios of data to the total prediction, uncertainty bars are statistical only. All uncertainties, which can be correlated across bins, are included in the error bands (hatched regions)

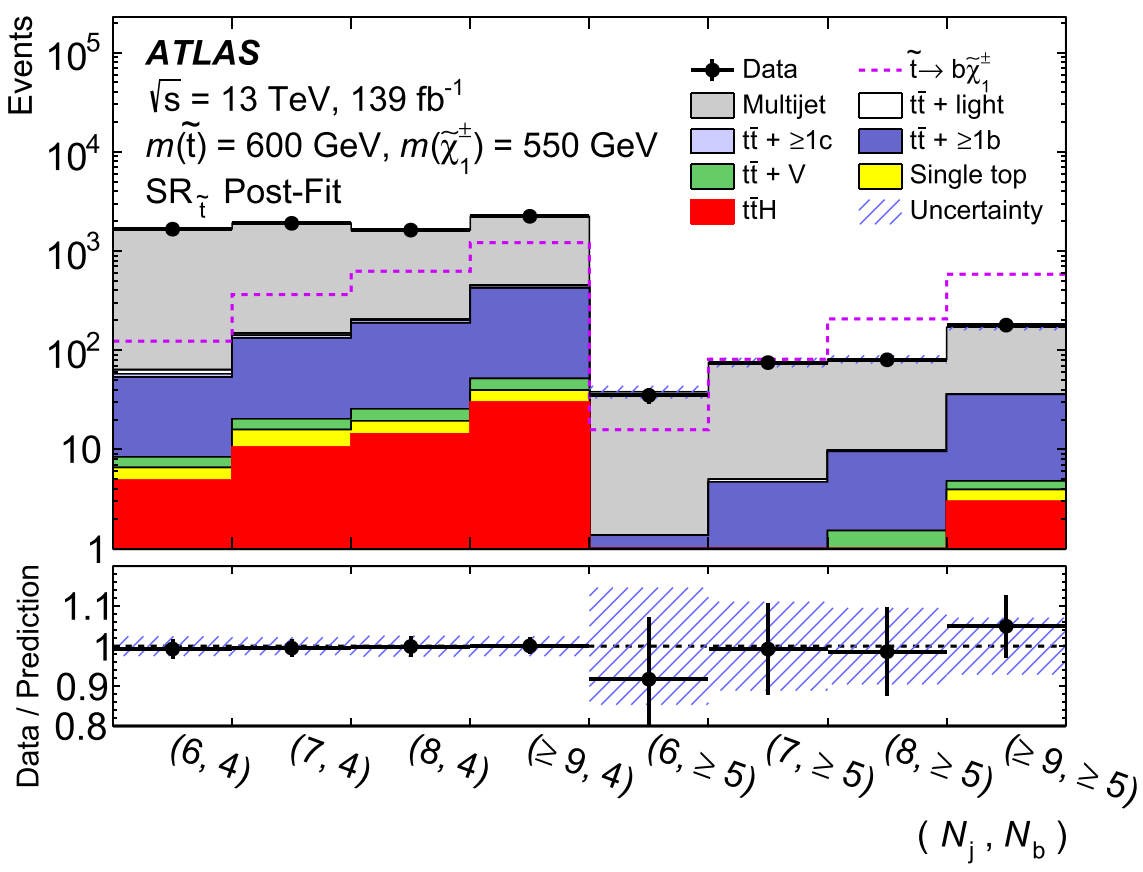

8.2 Model-dependent interpretation

Table 5 Fitted background yields in $\left(N_{\mathrm{j}} \geq 8, N_{\mathrm{b}} \geq 5\right)$ and $\left(N_{\mathrm{j}} \geq 9\right.$, $N_{\mathrm{b}} \geq 5$ ) signal regions. The individual background uncertainties can be larger than the total uncertainty due to correlations between parameters

\begin{tabular}{lll}
\hline Process & $N_{\mathrm{j}} \geq 8, N_{\mathrm{b}} \geq 5$ & $N_{\mathrm{j}} \geq 9, N_{\mathrm{b}} \geq 5$ \\
\hline Multijet & $200 \pm 40$ & $123 \pm 20$ \\
$t \bar{t}+\geq 1 c$ & $0.6 \pm 0.6$ & $0.29 \pm 0.33$ \\
$t \bar{t}+\geq 1 b$ & $26 \pm 20$ & $20 \pm 15$ \\
$t \bar{t}+W$ & $0.11 \pm 0.05$ & $0.09 \pm 0.04$ \\
$t \bar{t}+Z$ & $1.4 \pm 0.7$ & $0.8 \pm 0.7$ \\
$W t$ & $0.9 \pm 0.8$ & $0.9 \pm 1.2$ \\
$t \bar{t} H$ & $3.7 \pm 1.6$ & $2.9 \pm 1.4$ \\
\hline Total background & $230 \pm 40$ & $147 \pm 20$ \\
Data & 259 & 179 \\
\hline
\end{tabular}

Model-independent 95\% CL upper limits on the expected and observed number of BSM events, $N_{\exp }^{95}$ and $\sigma_{\text {obs }}^{95}$, that may contribute to the signal regions are computed from the observed number of events and the fitted background. Normalising these results by the integrated luminosity, $L$, of the data sample, allows them to be interpreted as upper limits on the visible BSM cross-section $\sigma_{\mathrm{obs}}^{95}$, defined as:

$\sigma_{\mathrm{obs}}^{95}=\sigma_{\mathrm{prod}} \times \mathrm{A} \times \epsilon=\frac{N_{\mathrm{obs}}^{95}}{L}$,

where $\sigma_{\text {prod }}$ is the production cross-section. The resulting limits are presented in Table 6. In addition, the $p_{0}$ values, which quantify the probability that a background-only hypothesis results in a fluctuation giving an event yield equal to or larger than the one observed in the data, are calculated, as are the corresponding Gaussian significance values $Z$.
For each signal model probed, the fit is configured using the model-dependent set-up, as detailed in the first part of Sect. 8 . Figure 7 shows exclusion limits at the $95 \%$ confidence level in the top-squark production model when $B\left(\tilde{t} \rightarrow b \tilde{\chi}_{1}^{+}\right)$is assumed to be unity. For this model, top-squark masses are excluded up to $950 \mathrm{GeV}$ for chargino masses close to the kinematic threshold for producing this final state. For lower values of the chargino mass, the limit weakens such that for chargino masses of around $200 \mathrm{GeV}$, the top-squark mass is constrained to be more than $800 \mathrm{GeV}$. In this phase space region, the signal is concentrated at lower $N_{\mathrm{j}}$ and $N_{\mathrm{b}}$ values where the background is larger.

The limits for higgsino LSPs are shown in Fig. 8. In the region $m_{\tilde{t}}-m_{\tilde{\chi}_{1,2}^{0}, \tilde{\chi}_{1}^{ \pm}} \geq m_{\text {top }}$ the sensitivity of the analysis is lower than in the pure $\tilde{t} \rightarrow b \tilde{\chi}_{1}^{ \pm}$case because contributions to the signal that have one leptonically decaying top quark fail the lepton-veto requirement. The large contribution of the multijet background reduces the present sensitivity relative to a previous ATLAS search that analysed events characterised by the presence of a lepton plus jets [11].

\section{Conclusion}

A search for physics beyond the Standard Model in events with high jet multiplicity and a large number of $b$-tagged jets is described in this paper. The search uses $139 \mathrm{fb}^{-1}$ of $\sqrt{s}=13 \mathrm{TeV}$ proton-proton collision data collected by the ATLAS experiment at the LHC. In contrast to many previous 


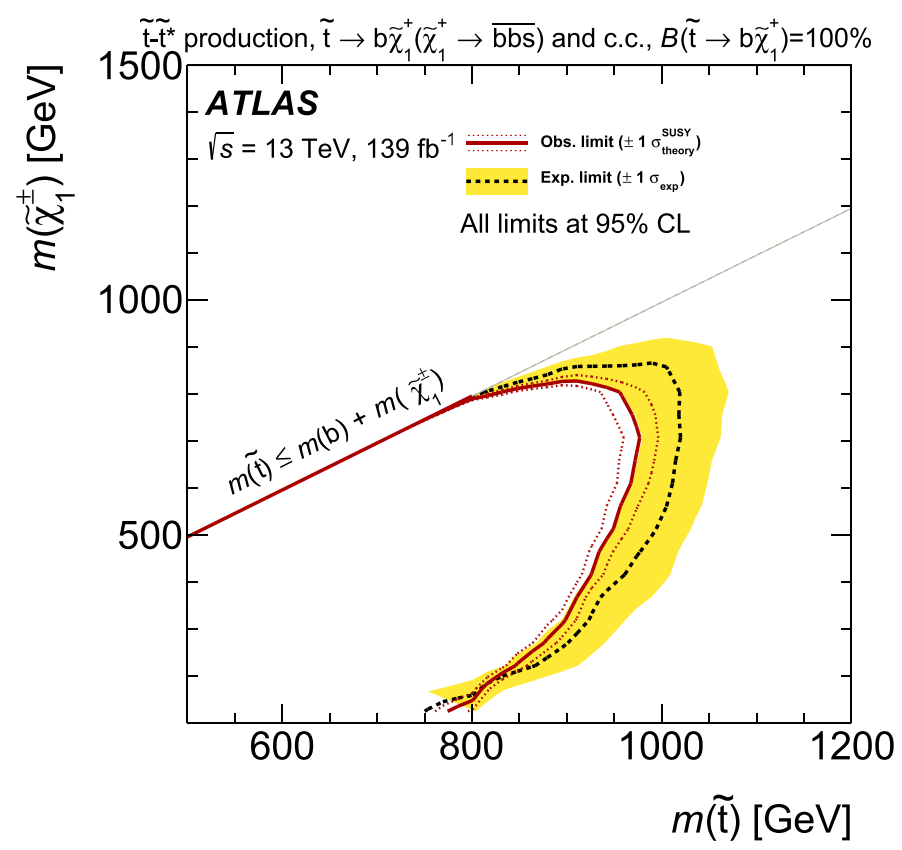

Fig. 7 Observed and expected exclusion contours for the $\tilde{t}$ and $\chi_{1}^{ \pm}$ masses in a top-squark production model with RPV decays of the $\chi_{1}^{ \pm}$. Limits are shown for $B\left(\tilde{t} \rightarrow b \chi_{1}^{+}\right)$equal to unity. The contours of the band around the expected limit are the $\pm 1 \sigma$ variations, including all uncertainties except theoretical uncertainties in the signal cross-section.
The dotted lines around the observed limit illustrate the change in the observed limit as the nominal signal cross-section is scaled up and down by the theoretical uncertainty. All limits are computed at 95\% CL. The results are constrained by the kinematic limits of the top-squark decay into a chargino and a bottom quark (diagonal line)

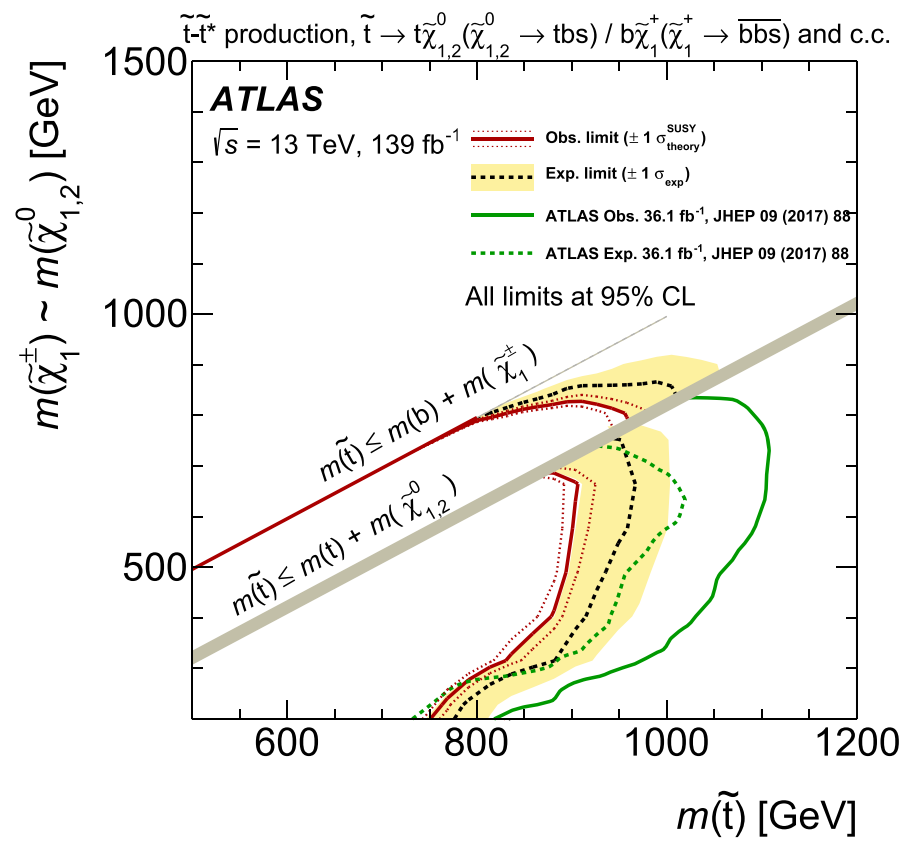

Fig. 8 Observed and expected exclusion contours for the $\tilde{t}$ and $\chi_{1}^{ \pm}$ masses in a top-squark production model with RPV decays of the $\chi_{1}^{ \pm}$ and of the $\tilde{\chi}_{1,2}^{0}$. Limits are shown in the case of a higgsino LSP. The contours of the band around the expected limit are the $\pm 1 \sigma$ variations, including all uncertainties except theoretical uncertainties in the signal cross-section. The dotted lines around the observed limit illustrate the change in the observed limit as the nominal signal cross-section is scaled up and down by the theoretical uncertainty. All limits are computed at 95\% CL. The results are constrained by the kinematic limits of the top-squark decay into a chargino and a bottom quark (upper diagonal line) and into a neutralino and a top quark (lower diagonal line), respectively. Also shown are the limits from Ref. [11] 
Table 6 Observed 95\% CL model-independent upper limits on the visible BSM cross-section, $\sigma_{\text {obs }}^{95}$, expressed in fb, along with the observed (expected) limits, $N_{\mathrm{obs}}^{95}\left(N_{\mathrm{exp}}^{95}\right)$, on the number of excess events. The limits are determined for two signal regions, $\left(N_{\mathrm{j}} \geq 8, N_{\mathrm{b}} \geq 5\right)$ and

\begin{tabular}{llrll}
\hline Signal region & $\sigma_{\text {obs }}^{95}[\mathrm{fb}]$ & $N_{\text {obs }}^{95}$ & $N_{\exp }^{95}$ & $p_{0}(Z)$ \\
\hline$N_{\mathrm{j}} \geq 8, N_{\mathrm{b}} \geq 5$ & 0.76 & 105 & $85_{-24}^{+30}$ & $0.24(0.7)$ \\
$N_{\mathrm{j}} \geq 9, N_{\mathrm{b}} \geq 5$ & 0.54 & 75 & $52_{-15}^{+20}$ & $0.11(1.2)$ \\
\hline
\end{tabular}

$\left(N_{\mathrm{j}} \geq 9, N_{\mathrm{b}} \geq 5\right)$. The $p_{0}$ value quantifies the probability that the background-only hypothesis would result in a fluctuation that gives an event yield equal to or larger than the one observed in the data, and $Z$ is the corresponding Gaussian significance searches in similar final states, leptons are vetoed and no requirement is placed on the missing transverse momentum in the event. The multijet background dominates the observed yields and is estimated using a data-driven technique based on an extrapolation from events with low $b$-jet multiplicity to the high $b$-jet multiplicities. No significant excess over the SM expectation is observed, and model-independent limits on the contribution of new phenomena to the signal-region yields are computed. In the context of a model with direct topsquark production and RPV decays of the higgsinos, the data exclude top squarks with masses up to $950 \mathrm{GeV}$ in the region $m_{\tilde{t}}-m_{\tilde{\chi}_{1,2}^{0}, \tilde{\chi}_{1}^{ \pm}} \leq m_{\text {top }}$, where this analysis has exclusive sensitivity. The results represent the first limits from ATLAS on the production of top squarks that decay exclusively into a chargino and a $b$-quark.

Acknowledgements We would like to thank Gilbert Moultaka for helpful discussions on the phenomenological aspects of the analysis. We thank CERN for the very successful operation of the LHC, as well as the support staff from our institutions without whom ATLAS could not be operated efficiently.

We acknowledge the support of ANPCyT, Argentina; YerPhI, Armenia; ARC, Australia; BMWFW and FWF, Austria; ANAS, Azerbaijan; SSTC, Belarus; CNPq and FAPESP, Brazil; NSERC, NRC and CFI, Canada; CERN; ANID, Chile; CAS, MOST and NSFC, China; COLCIENCIAS, Colombia; MSMT CR, MPO CR and VSC CR, Czech Republic; DNRF and DNSRC, Denmark; IN2P3-CNRS and CEA-DRF/IRFU, France; SRNSFG, Georgia; BMBF, HGF and MPG, Germany; GSRT, Greece; RGC and Hong Kong SAR, China; ISF and Benoziyo Center, Israel; INFN, Italy; MEXT and JSPS, Japan; CNRST, Morocco; NWO, Netherlands; RCN, Norway; MNiSW and NCN, Poland; FCT, Portugal; MNE/IFA, Romania; MES of Russia and NRC KI, Russia Federation; JINR; MESTD, Serbia; MSSR, Slovakia; ARRS and MIZŠ, Slovenia; DST/NRF, South Africa; MICINN, Spain; SRC and Wallenberg Foundation, Sweden; SERI, SNSF and Cantons of Bern and Geneva, Switzerland; MOST, Taiwan; TAEK, Turkey; STFC, United Kingdom; DOE and NSF, United States of America. In addition, individual groups and members have received support from BCKDF, CANARIE, Compute Canada and CRC, Canada; ERC, ERDF, Horizon 2020, Marie Skłodowska-Curie Actions and COST, European Union; Investissements d'Avenir Labex, Investissements d'Avenir Idex and ANR, France; DFG and AvH Foundation, Germany; Herakleitos, Thales and Aristeia programmes co-financed by EU-ESF and the Greek NSRF, Greece; BSF-NSF and GIF, Israel; La Caixa Banking Foundation, CERCA Programme Generalitat de Catalunya and PROMETEO and GenT Programmes Generalitat Valenciana, Spain; Göran Gustafssons Stiftelse, Sweden; The Royal Society and Leverhulme Trust, United Kingdom.
The crucial computing support from all WLCG partners is acknowledged gratefully, in particular from CERN, the ATLAS Tier-1 facilities at TRIUMF (Canada), NDGF (Denmark, Norway, Sweden), CCIN2P3 (France), KIT/GridKA (Germany), INFN-CNAF (Italy), NLT1 (Netherlands), PIC (Spain), ASGC (Taiwan), RAL (UK) and BNL (USA), the Tier- 2 facilities worldwide and large non-WLCG resource providers. Major contributors of computing resources are listed in Ref. [70].

Data Availability Statement This manuscript has no associated data or the data will not be deposited. [Authors' comment: All ATLAS scientific output is published in journals, and preliminary results are made available in Conference Notes. All are openly available, without restriction on use by external parties beyond copyright law and the standard conditions agreed by CERN. Data associated with journal publications are also made available: tables and data from plots (e.g. cross section values, likelihood profiles, selection efficiencies, cross section limits, ...) are stored in appropriate repositories such as HEPDATA (http:// hepdata.cedar.ac.uk/). ATLAS also strives to make additional material related to the paper available that allows a reinterpretation of the data in the context of new theoretical models. For example, an extended encapsulation of the analysis is often provided for measurements in the framework of RIVET (http://rivet.hepforge.org/)." This information is taken from the ATLAS Data Access Policy, which is a public document that can be downloaded from http://opendata.cern.ch/record/413 [opendata.cern.ch].]

Open Access This article is licensed under a Creative Commons Attribution 4.0 International License, which permits use, sharing, adaptation, distribution and reproduction in any medium or format, as long as you give appropriate credit to the original author(s) and the source, provide a link to the Creative Commons licence, and indicate if changes were made. The images or other third party material in this article are included in the article's Creative Commons licence, unless indicated otherwise in a credit line to the material. If material is not included in the article's Creative Commons licence and your intended use is not permitted by statutory regulation or exceeds the permitted use, you will need to obtain permission directly from the copyright holder. To view a copy of this licence, visit http://creativecomm ons.org/licenses/by/4.0/.

Funded by SCOAP ${ }^{3}$.

\section{References}

1. J.A. Evans, A swarm of Bs. JHEP 1408, 073 (2014). arXiv:1402.4481 [hep-ph]

2. M.R. Buckley, D. Feld, S. Macaluso, A. Monteux, D. Shih, Cornering natural SUSY at LHC Run II and beyond. JHEP 08, 115 (2017). arXiv:1610.08059 [hep-ph] 
3. S. Diglio, L. Feligioni, G. Moultaka, Stashing the stops in multijet events at the LHC. Phys. Rev. D 96, 055032 (2017). arXiv:1611.05850 [hep-ph]

4. P. Fayet, Supergauge invariant extension of the Higgs mechanism and a model for the electron and its neutrino. Nucl. Phys. B 90, 104 (1975)

5. A. Salam, J.A. Strathdee, Supersymmetry and fermion number conservation. Nucl. Phys. B 87, 85 (1975)

6. R. Barbieri et al., R-parity violating supersymmetry. Phys. Rep. 420, 1 (2005). arXiv:hep-ph/0406039

7. R. Barbieri, G. Giudice, Upper bounds on supersymmetric particle masses. Nucl. Phys. B 306, 63 (1988)

8. B. de Carlos, J. Casas, One-loop analysis of the electroweak breaking in supersymmetric models and the fine-tuning problem. Phys. Lett. B 309, 320 (1993). arXiv:hep-ph/9303291

9. G. D'Ambrosio, G. Giudice, G. Isidori, A. Strumia, Minimal flavour violation: an effective field theory approach. Nucl. Phys. B 645, 155 (2002). ISSN:0550-3213. https://doi.org/10.1016/ S0550-3213(02)00836-2

10. C. Csáki, Y. Grossman, B. Heidenreich, Minimal flavor violation supersymmetry: a natural theory for R-parity violation. Phys. Rev. D 85 (2012). ISSN:1550-2368. http://dx.doi.org/10.1103/ PhysRevD.85.095009

11. ATLAS Collaboration, Search for new phenomena in a lepton plus high jet multiplicity final state with the ATLAS experiment using $\sqrt{s}=13 \mathrm{TeV}$ proton-proton collision data. JHEP 09, 088 (2017). arXiv:1704.08493 [hep-ex]

12. ATLAS Collaboration, A search for pair-produced resonances in four-jet final states at $\sqrt{s}=13 \mathrm{TeV}$ with the ATLAS detector. Eur. Phys. J. C 78, 250 (2018). arXiv:1710.07171 [hep-ex]

13. CMS Collaboration, Search for pair-produced resonances decaying to quark pairs in proton-proton collisions at $\sqrt{s}=13 \mathrm{TeV}$. Phys. Rev. D 98, 112014 (2018). arXiv:1808.03124 [hep-ex]

14. CMS Collaboration, Search for pair-produced resonances each decaying into at least four quarks in proton-proton collisions at $\sqrt{s}=13$ TeV. Phys. Rev. Lett. 121, 141802 (2018). arXiv: 1806.01058 [hep-ex]

15. ATLAS Collaboration, The ATLAS experiment at the CERN Large Hadron Collider, JINST 3, S08003 (2008)

16. ATLAS Collaboration, ATLAS insertable B-layer technical design report, ATLAS-TDR-19 (2010). https://cds.cern.ch/record/ 1291633

17. B. Abbott et al., Production and integration of the ATLAS Insertable B-Layer. JINST 13, T05008 (2018). arXiv:1803.00844 [physics.ins-det]

18. ATLAS Collaboration, Luminosity determination in pp collisions at $\sqrt{s}=13 \mathrm{TeV}$ using the ATLAS detector at the LHC, ATLASCONF-2019-021 (2019). https://cds.cern.ch/record/2677054

19. G. Avoni et al., The new LUCID-2 detector for luminosity measurement and monitoring in ATLAS. J. Instrum. 13, P07017 (2018)

20. T. Sjöstrand et al., An introduction to PYTHIA 8.2. Comput. Phys. Commun. 191, 159 (2015). arXiv:1410.3012 [hep-ph]

21. The Pythia 8 A3 tune description of ATLAS minimum bias and inelastic measurements incorporating the Donnachie-Landshoff diffractive model, Tech. Rep. ATL-PHYS-PUB-2016-017, CERN (2016). https://cds.cern.ch/record/2206965

22. R.D. Ball et al., Parton distributions with LHC data. Nucl. Phys. B 867, 244 (2013). arXiv:1207.1303 [hep-ph]

23. ATLAS Collaboration, The ATLAS simulation infrastructure. Eur. Phys. J. C 70, 823 (2010). arXiv:1005.4568 [physics.ins-det]

24. GEANT4 Collaboration, S. Agostinelli et al., GEANT4: a simulation toolkit. Nucl. Instrum. Methods A 506, 250 (2003)

25. W. Lukas, Fast Simulation for ATLAS: Atlfast-II and ISF, Tech. Rep. ATL-SOFT-PROC-2012-065, CERN (2012). https://cds.cern. $\mathrm{ch} / \mathrm{record} / 1458503$
26. ATLAS Collaboration, ATLAS Pythia 8 tunes to $7 \mathrm{TeV}$ data, ATLPHYS-PUB-2014-021 (2014). https://cds.cern.ch/record/1966419

27. D.J. Lange, The EvtGen particle decay simulation package. Nucl. Instrum. Methods A 462, 152 (2001)

28. ATLAS Collaboration, Jet energy scale measurements and their systematic uncertainties in proton-proton collisions at $\sqrt{s}=13$ TeV with the ATLAS detector. Phys. Rev. D 96, 072002 (2017). arXiv:1703.09665 [hep-ex]

29. ATLAS Collaboration, ATLAS b-jet identification performance and efficiency measurement with $t \bar{t}$ events in pp collisions at $\sqrt{s}=13$ TeV. Eur. Phys. J. C 79, 970 (2019). arXiv:1907.05120 [hep-ex]

30. S. Frixione, P. Nason, G. Ridolfi, A positive-weight next-toleading-order Monte Carlo for heavy flavour hadroproduction. JHEP 09, 126 (2007). arXiv:0707.3088 [hep-ph]

31. P. Nason, A new method for combining NLO QCD with shower Monte Carlo algorithms. JHEP 11, 040 (2004). arXiv:hep-ph/0409146

32. S. Frixione, P. Nason, C. Oleari, Matching NLO QCD computations with Parton Shower simulations: the POWHEG method. JHEP 11, 070 (2007). arXiv:0709.2092 [hep-ph]

33. S. Alioli, P. Nason, C. Oleari, E. Re, A general framework for implementing NLO calculations in shower Monte Carlo programs: the POWHEG BOX. JHEP 06, 043 (2010). arXiv:1002.2581 [hep$\mathrm{ph}]$

34. NNPDF Collaboration, R.D. Ball et al., Parton distributions for the LHC Run II. JHEP 04, 040 (2015). arXiv:1410.8849 [hep-ph]

35. ATLAS Collaboration, Studies on top-quark Monte Carlo modelling for Top2016, ATL-PHYS-PUB-2016-020 (2016). https:// cds.cern.ch/record/2216168

36. M. Beneke, P. Falgari, S. Klein, C. Schwinn, Hadronic top-quark pair production with NNLL threshold resummation. Nucl. Phys. B 855, 695 (2012). arXiv:1109.1536 [hep-ph]

37. M. Cacciari, M. Czakon, M. Mangano, A. Mitov, P. Nason, Toppair production at hadron colliders with next-to-next-to-leading logarithmic soft-gluon resummation. Phys. Lett. B 710, 612 (2012). arXiv:1111.5869 [hep-ph]

38. P. Bärnreuther, M. Czakon, A. Mitov, Percent-level-precision physics at the Tevatron: next-to-next-to-leading order QCD corrections to $q \bar{q} \rightarrow t \bar{t}+X$. Phys. Rev. Lett. 109, 132001 (2012). arXiv:1204.5201 [hep-ph]

39. M. Czakon, A. Mitov, NNLO corrections to top-pair production at hadron colliders: the all-fermionic scattering channels. JHEP 12, 054 (2012). arXiv:1207.0236 [hep-ph]

40. M. Czakon, A. Mitov, NNLO corrections to top pair production at hadron colliders: the quark-gluon reaction. JHEP 01, 080 (2013). arXiv:1210.6832 [hep-ph]

41. M. Czakon, P. Fiedler, A. Mitov, Total top-quark pair-production cross section at hadron colliders through $O\left(\alpha_{S}^{4}\right)$. Phys. Rev. Lett. 110, 252004 (2013). arXiv:1303.6254 [hep-ph]

42. M. Czakon, A. Mitov, Top++: a program for the calculation of the top-pair cross-section at hadron colliders. Comput. Phys. Commun. 185, 2930 (2014). arXiv:1112.5675 [hep-ph]

43. M. Cacciari, G.P. Salam, G. Soyez, The anti- $\mathrm{k}_{t}$ jet clustering algorithm. JHEP 04, 063 (2008). arXiv:0802.1189 [hep-ph]

44. S. Frixione, E. Laenen, P. Motylinski, B.R. Webber, C.D. White, Single-top hadroproduction in association with a $\mathrm{W}$ boson. JHEP 07, 029 (2008). arXiv:0805.3067 [hep-ph]

45. N. Kidonakis, Two-loop soft anomalous dimensions for single top quark associated production with a $\mathrm{W}-$ or $\mathrm{H}-$. Phys. Rev. D 82, 054018 (2010). arXiv: 1005.4451 [hep-ph]

46. N. Kidonakis, 'Top Quark Production', Proceedings, Helmholtz International Summer School on Physics of Heavy Quarks and Hadrons (HQ 2013): JINR, Dubna, Russia, July 15-28, 2013 (2014). arXiv:1311.0283 [hep-ph] 
47. J. Alwall et al., The automated computation of tree-level and nextto-leading order differential cross sections, and their matching to parton shower simulations. JHEP 07, 079 (2014). arXiv:1405.0301 [hep-ph]

48. D. de Florian et al., Handbook of LHC Higgs Cross Sections: 4. Deciphering the Nature of the Higgs Sector (2016). arXiv:1610.07922 [hep-ph]

49. W. Beenakker, C. Borschensky, M. Kramer, A. Kulesza, E. Laenen, NNLL-fast: predictions for coloured supersymmetric particle production at the LHC with threshold and Coulomb resummation. JHEP 12, 133 (2016). arXiv:1607.07741 [hep-ph]

50. ATLAS Collaboration, Electron and photon energy calibration with the ATLAS detector using LHC Run 1 data. Eur. Phys. J. C 74 (2014). arXiv:1407.5063 [hep-ex]

51. ATLAS Collaboration, Electron efficiency measurements with the ATLAS detector using the 2015 LHC proton-proton collision data, ATLAS-CONF-2016-024 (2016). https://cds.cern.ch/record/ 2157687

52. ATLAS Collaboration, Electron and photon performance measurements with the ATLAS detector using the 2015-2017 LHC protonproton collision data. JINST 14, P12006 (2019). arXiv:1908.00005 [hep-ex]

53. ATLAS Collaboration, Muon reconstruction performance of the ATLAS detector in proton-proton collision data at $\sqrt{s}=13 \mathrm{TeV}$. Eur. Phys. J. C 76, 292 (2016). arXiv:1603.05598 [hep-ex]

54. W. Lampl et al., Calorimeter Clustering Algorithms: Description and Performance, Tech. Rep. ATL-LARG-PUB-2008-002. ATLCOM-LARG-2008-003, CERN (2008). https://cds.cern.ch/record/ 1099735

55. ATLAS Collaboration, Jet energy scale and resolution measured in proton-proton collisions at $\sqrt{s}=13 \mathrm{TeV}$ with the ATLAS detector (2020). arXiv:2007.02645 [hep-ex]

56. ATLAS Collaboration, Performance of pile-up mitigation techniques for jets in pp collisions at $\sqrt{s}=8 \mathrm{TeV}$ using the ATLAS detector. Eur. Phys. J. C 76, 581 (2016). arXiv: 1510.03823 [hepex]

57. ATLAS Collaboration, Selection of jets produced in $13 \mathrm{TeV}$ proton-proton collisions with the ATLAS detector, ATLASCONF-2015-029 (2015). http://cdsweb.cern.ch/record/2037702
58. ATLAS Collaboration, Optimisation of the ATLAS b-tagging performance for the 2016 LHC Run, Tech. Rep. ATL-PHYS-PUB2016-012, CERN (2016). https://cds.cern.ch/record/2160731

59. ATLAS Collaboration, Search for the Standard Model Higgs boson decaying into $b \bar{b}$ produced in association with top quarks decaying hadronically in pp collisions at $\sqrt{s}=8 \mathrm{TeV}$ with the ATLAS detector. JHEP 05, 160 (2016). arXiv:1604.03812 [hep-ex]

60. ATLAS Collaboration, Search for four-top-quark production in the single-lepton and opposite-sign dilepton final states in pp collisions at $\sqrt{s}=13 \mathrm{TeV}$ with the ATLAS detector. Phys. Rev. D 99, 052009 (2019). arXiv:1811.02305 [hep-ex]

61. D0 Collaboration, Measurement of the $t$ anti-t production cross section in $\mathrm{p}$ anti-p collisions at $\sqrt{s}=1.96 \mathrm{TeV}$ using secondary vertex b tagging. Phys. Rev. D 74, 112004 (2006). arXiv:hep-ex/0611002

62. ATLAS Collaboration, Search for the standard model Higgs boson produced in association with top quarks and decaying into a $b \bar{b}$ pair in pp collisions at $\sqrt{s}=13 \mathrm{TeV}$ with the ATLAS detector. Phys. Rev. D 97, 072016 (2018). arXiv:1712.08895 [hep-ex]

63. M. Bahr et al., Herwig++ physics and manual. Eur. Phys. J. C 58, 639 (2008). arXiv:0803.0883 [hep-ph]

64. J. Bellm et al., Herwig 7.0/Herwig ++ 3.0 release note. Eur. Phys. J. C 76, 196 (2016). arXiv:1512.01178 [hep-ph]

65. L. Harland-Lang, A. Martin, P. Motylinski, R. Thorne, Parton distributions in the LHC era: MMHT2014 PDFs. Eur. Phys. J. C 75, 204 (2015). arXiv:1412.3989 [hep-ph]

66. N. Kidonakis, Next-to-next-to-leading-order collinear and soft gluon corrections for t-channel single top quark production. Phys. Rev. D 83, 091503 (2011). arXiv:1103.2792 [hep-ph]

67. N. Kidonakis, NNLL resummation for s-channel single top quark production. Phys. Rev. D 81, 054028 (2010). arXiv:1001.5034 [hep-ph]

68. W. Verkerke, D. Kirkby, The RooFit toolkit for data modeling (2003). arXiv:physics/0306116 [physics.data-an]

69. A.L. Read, Presentation of search results: the CL(s) technique. J. Phys. G 28, 2693 (2002)

70. ATLAS Collaboration, ATLAS Computing Acknowledgements, ATL-SOFT-PUB-2020-001. https://cds.cern.ch/record/2717821 


\section{ATLAS Collaboration}

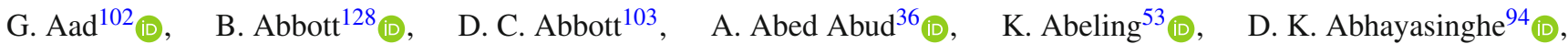

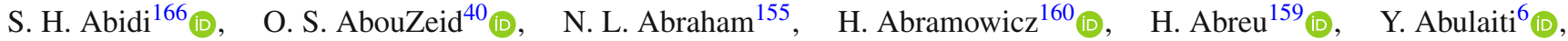

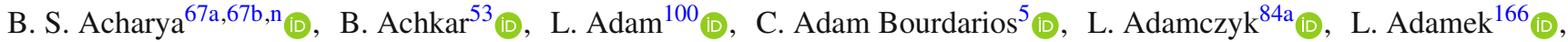
J. Adelman ${ }^{121}$ (I) , M. Adersberger ${ }^{114}$, A. Adiguzel ${ }^{12 c}$ (I), S. Adorni ${ }^{54}$ (D), T. Adye ${ }^{143}$ (D), A. A. Affolder ${ }^{145}$ (D), Y. Afik ${ }^{159}$ (D), C. Agapopoulou ${ }^{65}$ (D), M. N. Agaras ${ }^{38}$ (I) A. Aggarwal ${ }^{119}$ (D), C. Agheorghiesei ${ }^{27 c}$ (D), J. A. Aguilar-Saavedra ${ }^{139 f, 139 a, a d}$ (D),

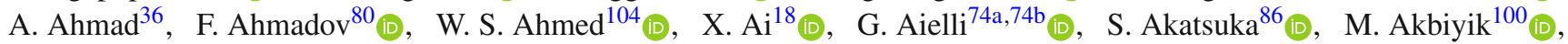
T. P. A. Åkesson ${ }^{97}$ (D), E. Akilli ${ }^{54}$ (D), A. V. Akimov ${ }^{111_{\text {(D) }}, \quad \text { K. Al Khoury }}{ }^{65}$ (D), G. L. Alberghi ${ }^{23 a, 23 b}$ (D), J. Albert ${ }^{175}$ (D),

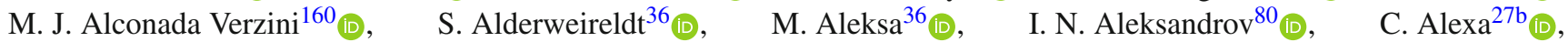

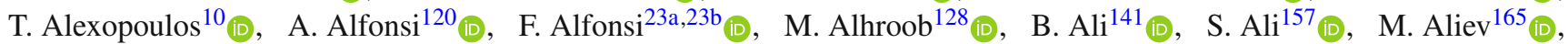

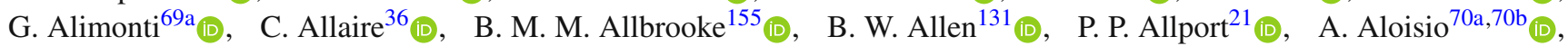

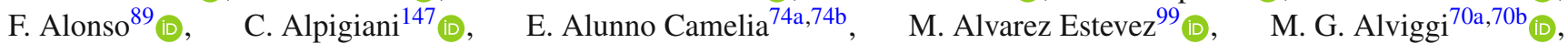

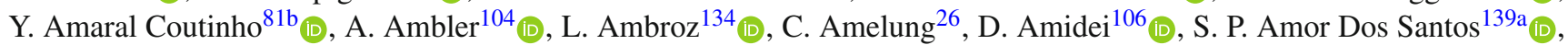

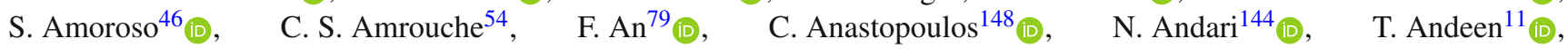

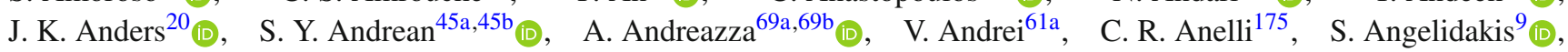

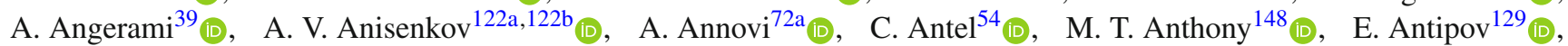

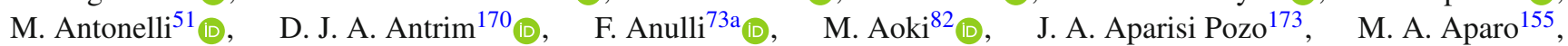

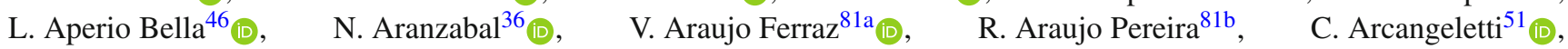

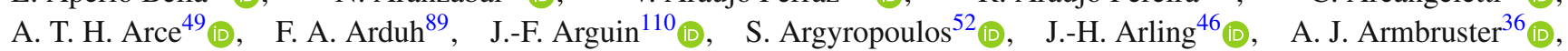

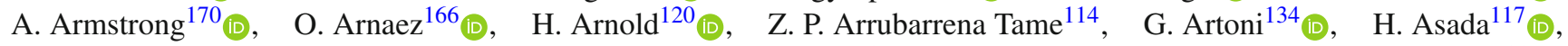
$\begin{array}{lll}\text { K. Asai } & 126\end{array}$ S. Asai ${ }^{162}$ (D), T. Asawatavonvanich ${ }^{164}, \quad$ N. Asbah ${ }^{59}$ (D), E. M. Asimakopoulou ${ }^{171}$ (D), L. Asquith ${ }^{155}$ (D),

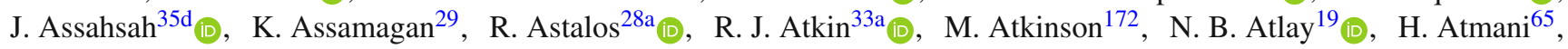
K. Augsten ${ }^{141}$ (D), V. A. Austrup ${ }^{181}$ (D), G. Avolio ${ }^{36}$ (D), M. K. Ayoub ${ }^{15 a}$ (D), G. Azuelos ${ }^{110, a l}$ (D), H. Bachacou ${ }^{144}$ (D), K. Bachas ${ }^{161}$ (D), F. Backman ${ }^{45 a, 45 b}$, P. Bagnaia ${ }^{73 a, 73 b}$ (D), M. Bahmani ${ }^{85}$ (D, H. Bahrasemani ${ }^{151}, \quad$ A. J. Bailey ${ }^{173}$ (D), V. R. Bailey ${ }^{172}$ (D) J.T. Baines ${ }^{143}$ (D) C. Bakalis ${ }^{10}$, O. K. Baker ${ }^{182}$ (D) P. J. Bakker ${ }^{120}$ (D), E. Bakos $^{16}$ (D) D. Bakshi Gupta ${ }^{8}$ (D),

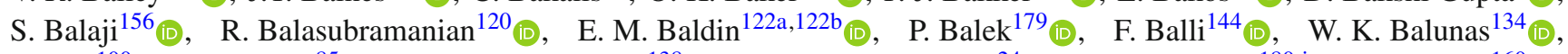
J. Balz ${ }^{100}$ (D), E. Banas ${ }^{85}$ (D), M. Bandieramonte ${ }^{138}$ (D), A. Bandyopadhyay ${ }^{24}$ (D), Sw. Banerjee ${ }^{\left.180, i_{(}\right), \quad \text { L. Barak }}{ }^{160}$ (D),

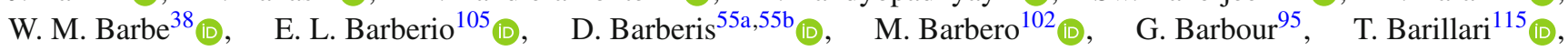

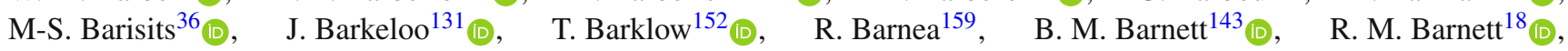

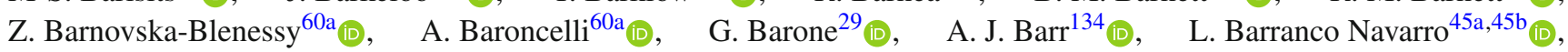

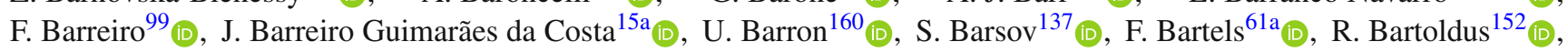

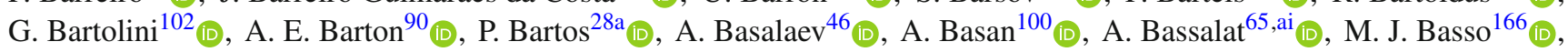

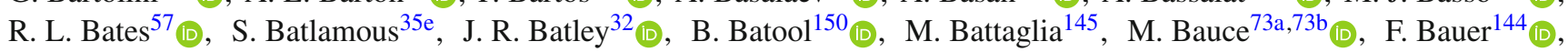

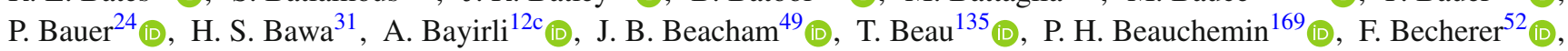

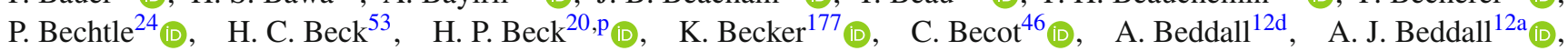

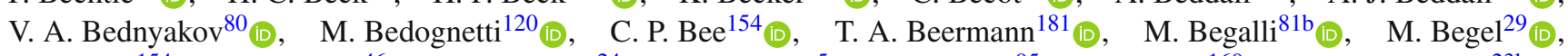

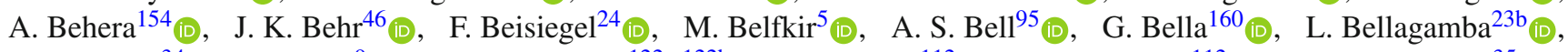

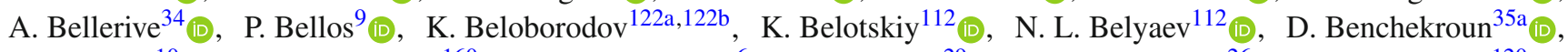

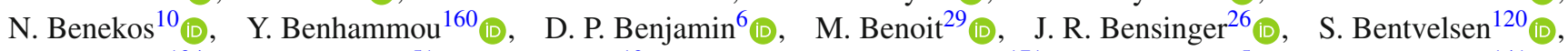

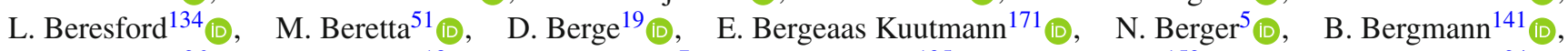

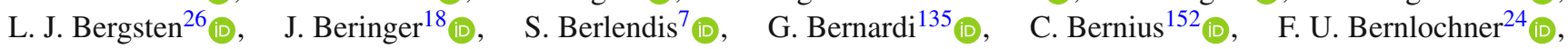

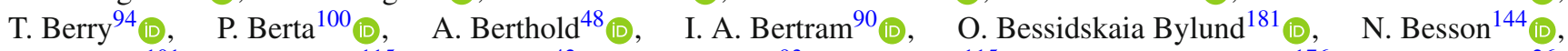
A. Bethani ${ }^{101}$ (I) S. Bethke ${ }^{115}$ (D), A. Betti ${ }^{42}$ (D) A. J. Bevan ${ }^{93}$ (D), J. Beyer ${ }^{115}$ (D), D. S. Bhattacharya ${ }^{176}$ (D), P. Bhattarai ${ }^{26}$,

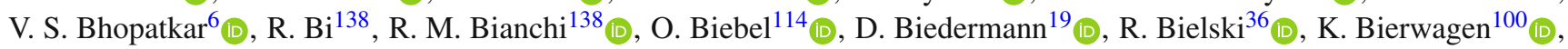

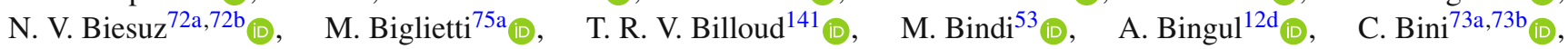

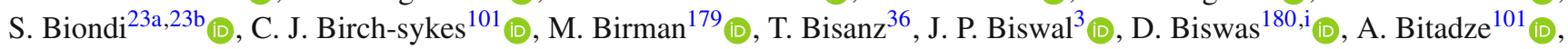
C. Bittrich ${ }^{48}$ (D), K. Bjørke ${ }^{133}$ (D), T. Blazek ${ }^{28 a}$ (D), I. Bloch ${ }^{46}$ (D), C. Blocker ${ }^{26}$ (D), A. Blue ${ }^{57}$ (D), U. Blumenschein ${ }^{93}$ (D),

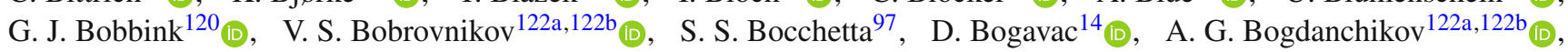

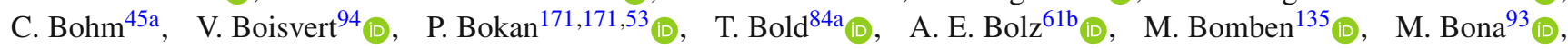
J. S. Bonilla ${ }^{131}$ (D) M. Boonekamp ${ }^{144}$ (D), C. D. Booth ${ }^{94}$, A. G. Borbély ${ }^{57}$ (D) H. M. Borecka-Bielska ${ }^{91}$ (D), L. S. Borgna ${ }^{95}$,

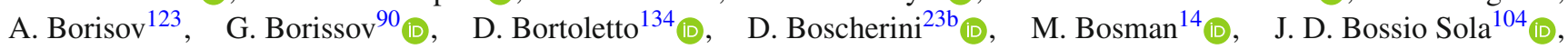
K. Bouaouda ${ }^{35 \mathrm{a}}$ (i), J. Boudreau ${ }^{138}$ (D), E. V. Bouhova-Thacker ${ }^{90}$ (D) D. Boumediene ${ }^{38}$ (D), A. Boveia ${ }^{127}$ (D), J. Boyd ${ }^{36}$ (D), 


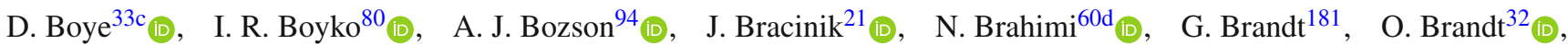
F. Braren ${ }^{46}$ (D) B. Brau ${ }^{103}$ (D) J. E. Brau ${ }^{131}$ (D), W. D. Breaden Madden $^{57}$, K. Brendlinger ${ }^{46}$, , R. Brener ${ }^{159}$ (D), L. Brenner ${ }^{36}$ (D), R. Brenner ${ }^{171}$ (D), S. Bressler ${ }^{179}$ (D), B. Brickwedde ${ }^{100}$ (D) D. L. Briglin ${ }^{21}$ (D), D. Britton ${ }^{57}$ (D), D. Britzger ${ }^{115}$ (I) I. Brock ${ }^{24}$ (D), R. Brock ${ }^{107}$ (D), G. Brooijmans ${ }^{39}$ (D) W. K. Brooks ${ }^{146 d}$ (D), E. Brost ${ }^{29}$ (D) P. A. Bruckman de Renstrom ${ }^{85}$ (D) B. Brüers ${ }^{46}{ }_{(\mathbb{D})}$,

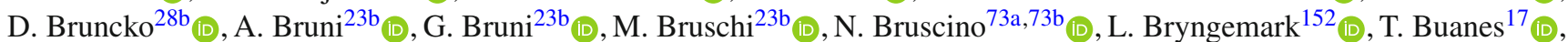

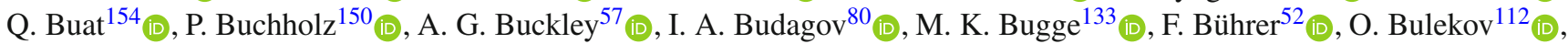

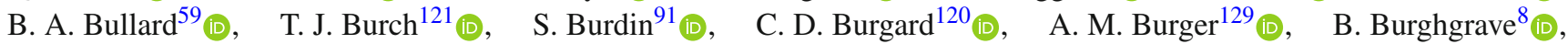
J. T. P. Burr ${ }^{46}$, C.D. Burton ${ }^{11}$ (D) J. C. Burzynski ${ }^{103}$, V. Büscher ${ }^{100}$ (D) E. Buschmann ${ }^{53}$, P. J. Bussey ${ }^{57}$ (D) J. M. Butler ${ }^{25}$ (D),

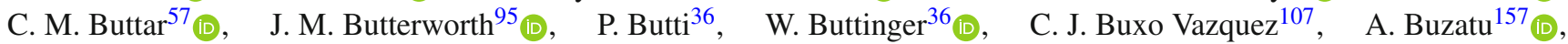

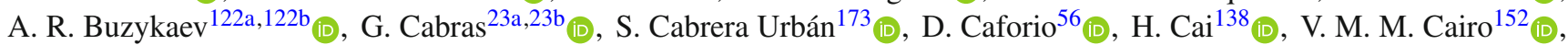

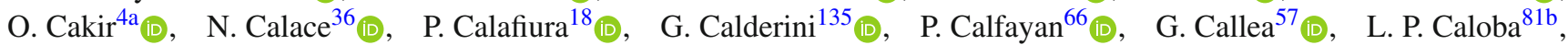
A. Caltabiano ${ }^{74 a, 74 b}$, S. Calvente Lopez $^{99}$ (D), D. Calvet ${ }^{38}$ (D), S. Calvet ${ }^{38}$ (D), T. P. Calvet ${ }^{102}$ (D), M. Calvetti ${ }^{72 a, 72 b}$ (D),

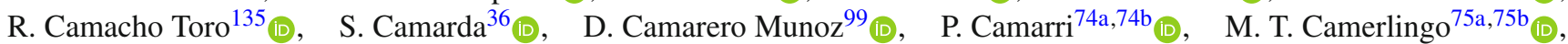
D. Cameron ${ }^{13}{ }_{\text {(D) }}, \quad$ C. Camincher $^{36}$ (D), S. Campana ${ }^{36}, \quad$ M. Campanelli ${ }^{95}$ (D), A. Camplani ${ }^{40}$ (D), V. Canale ${ }^{70 a, 70 b}{ }_{(\mathbb{D})}$,

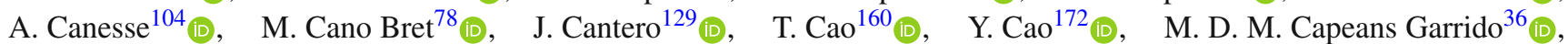

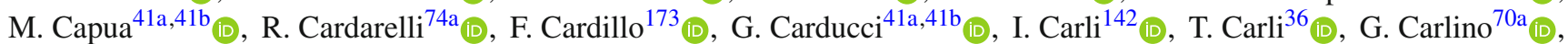

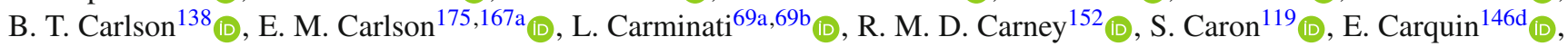

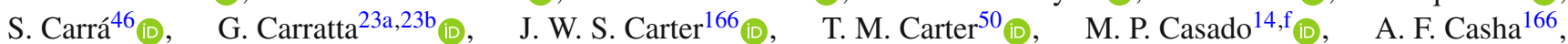

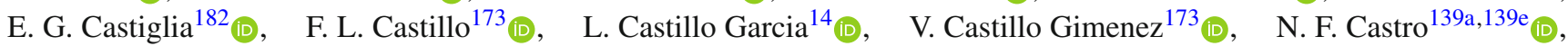
A. Catinaccio ${ }^{36}$ (D), J. R. Catmore ${ }^{133}$, A. Cattai ${ }^{36}$, V. Cavaliere ${ }^{29}$ (D), V. Cavasinni ${ }^{72 a, 72 b}$ (D), E. Celebi ${ }^{12 b}$, F. Celli ${ }^{134}$ (D),

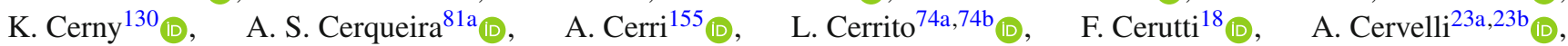

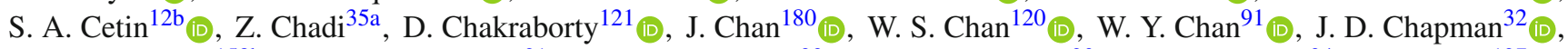

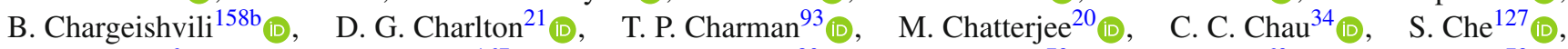

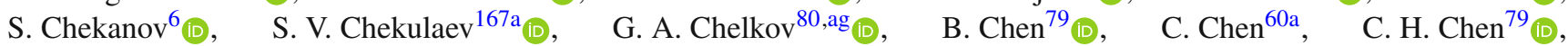

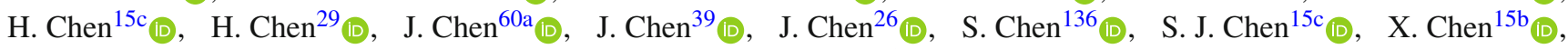

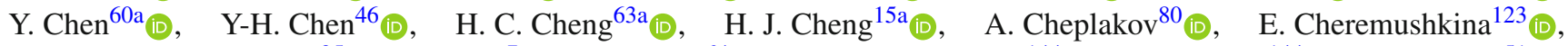

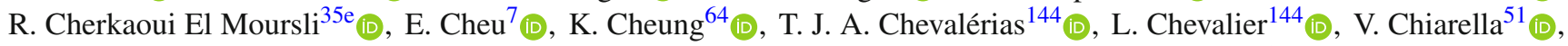

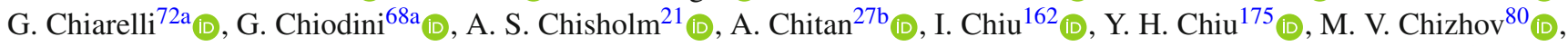

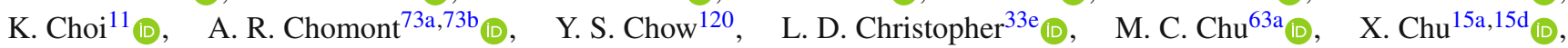
J. Chudoba ${ }^{140}$ (D) J. J. Chwastowski ${ }^{85}$ (D) L. Chytka ${ }^{130}$, D. Cieri ${ }^{115}$ (D) K. M. Ciesla ${ }^{85}$ (D) V. Cindro ${ }^{92}$ (D) I. A. Cioară ${ }^{27 b}$ (D),

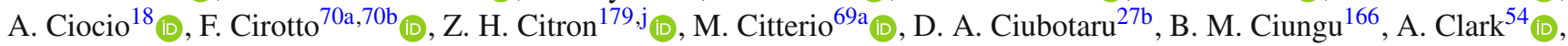
M. R. Clark ${ }^{39}$ (D), P. J. Clark ${ }^{50}$ (D) $\quad$ S. E. Clawson ${ }^{101}$ (I),$\quad$ C. Clement ${ }^{45 a, 45 b}$ (D), Y. Coadou ${ }^{102}$ (D), M. Cobal ${ }^{67 a, 67 c}$ (D),

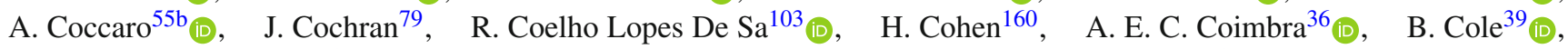
A. P. Colijn ${ }^{120}$, J. Collot $^{58}$ (D), P. Conde Muiño ${ }^{139 a}, 139 h_{\text {(D) }}$ S. H. Connell ${ }^{33 \mathrm{c}}$ (I), I. A. Connelly ${ }^{57}$ (D) S. Constantinescu ${ }^{27 b}$,

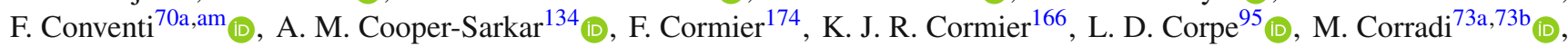

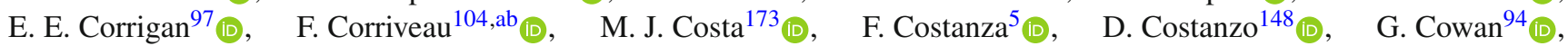
J. W. Cowley ${ }^{32}$ (D), J. Crane ${ }^{101}$ (D), K. Cranmer ${ }^{125}$ (D), R. A. Creager ${ }^{136}$ (D), S. Crépé-Renaudin ${ }^{58}$ (D), F. Crescioli ${ }^{135}$ (D),

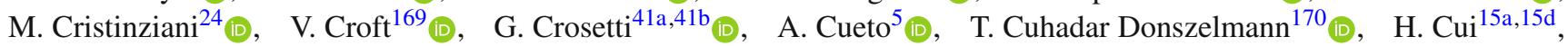

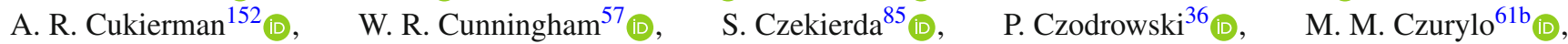

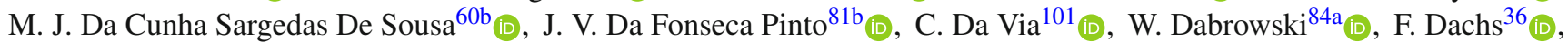

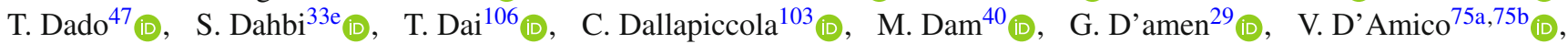

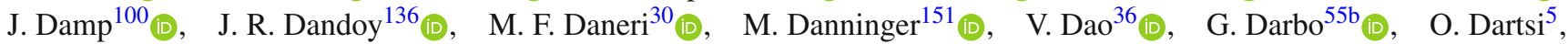

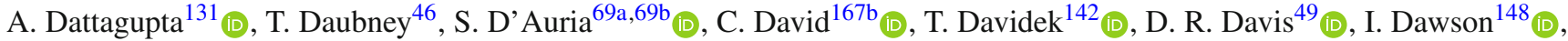

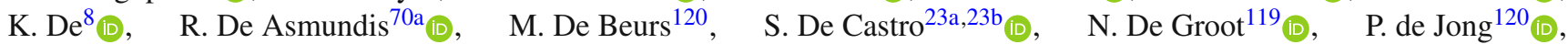

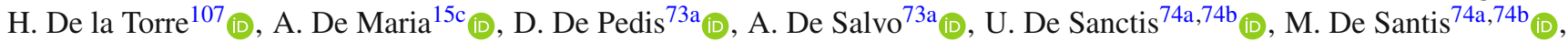
A. De Santo ${ }^{155}$ (D) J. B. De Vivie De Regie ${ }^{65}$ (D) D. V. Dedovich ${ }^{80}$, A. M. Deiana ${ }^{42}$ (D) J. Del Peso ${ }^{99}$ (D), Y. Delabat Diaz ${ }^{46}$ (D), D. Delgove ${ }^{65}$ (D), F. Deliot ${ }^{144}$ (D), C. M. Delitzsch ${ }^{7}$ (D), M. Della Pietra ${ }^{70 a, 70 b}$ (D), D. Della Volpe ${ }^{54}$ (D), A. Dell' Acqua $^{36}$ (D),

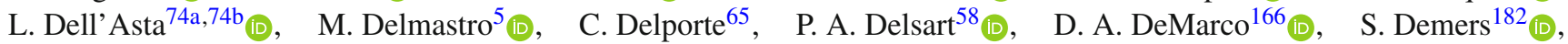
M. Demichev ${ }^{80}$ (D) G. Demontigny ${ }^{110}$, S. P. Denisov ${ }^{123}$ (D) , L. D’Eramo ${ }^{121}$ (D), D. Derendarz ${ }^{85}$ (D), J. E. Derkaoui ${ }^{35 d^{36}}$, F. Derue ${ }^{135}$ (D), P. Dervan ${ }^{91}$ (D), K. $\operatorname{Desch}^{24}$ (D) K. Dette ${ }^{166}$ (D) C. Deutsch ${ }^{24}$ (D), M. R. Devesa ${ }^{30}$, P. O. Deviveiros ${ }^{36}$ (D),

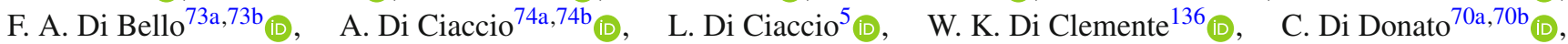

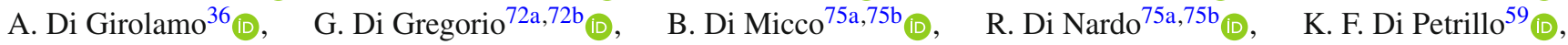

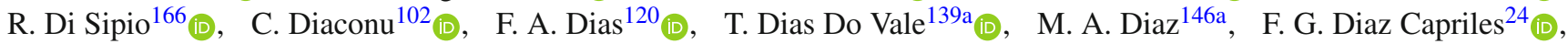




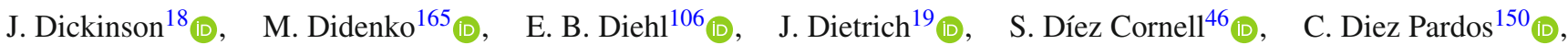

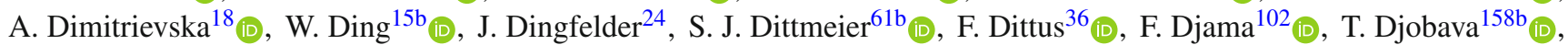

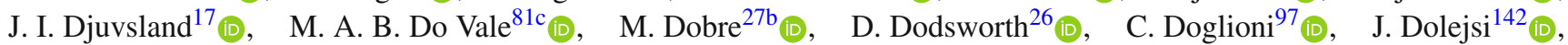

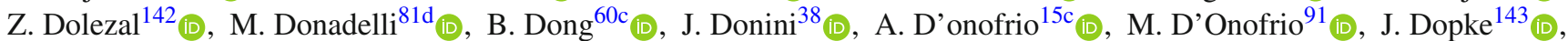

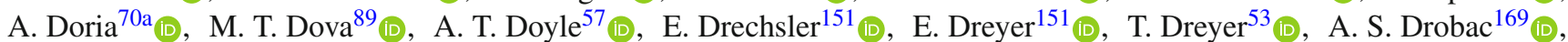

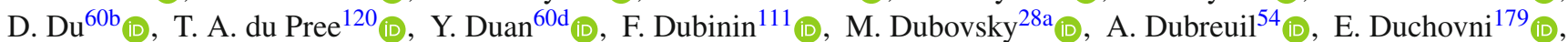

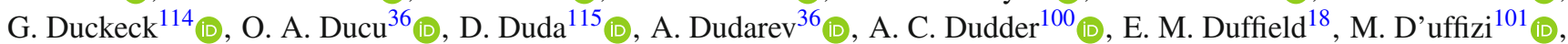

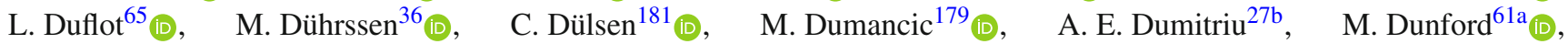

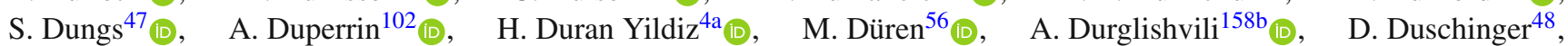

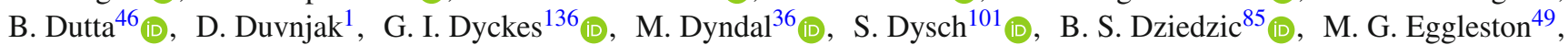

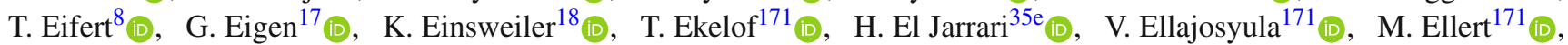

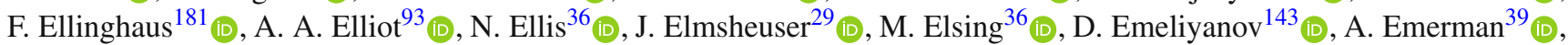

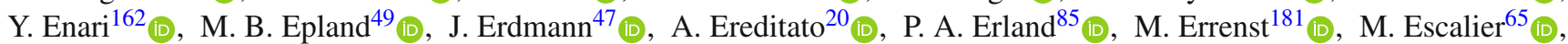

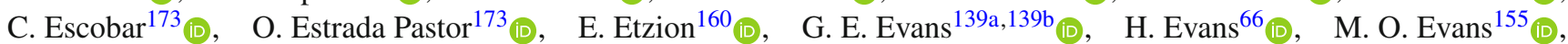

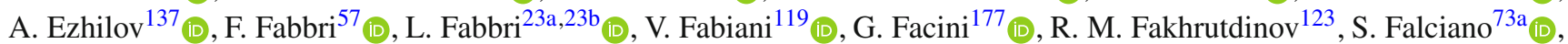

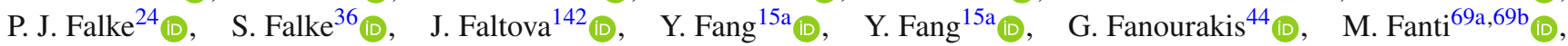

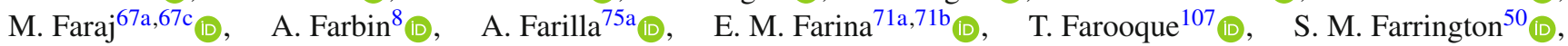

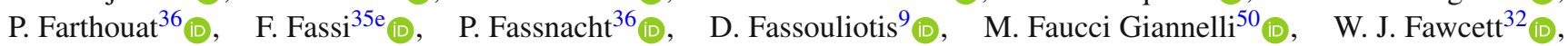

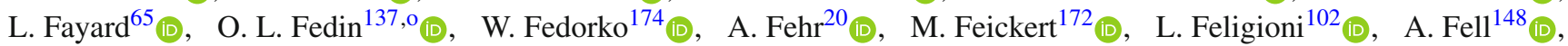

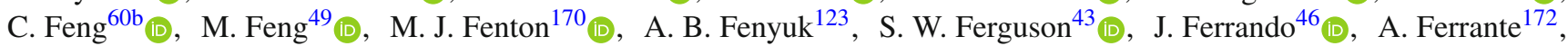

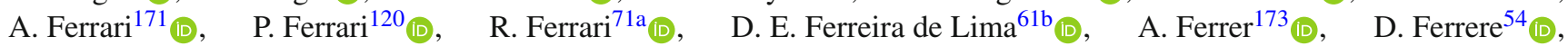

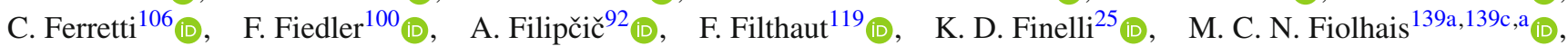
L. Fiorini ${ }^{173}$ (D), F. Fischer ${ }^{114}$ (D), J. Fischer ${ }^{100}$ (D), W. C. Fisher ${ }^{107}$ (D), T. Fitschen ${ }^{21}$ (D), I. Fleck ${ }^{150}$ (D) P. Fleischmann ${ }^{106}$ (D),

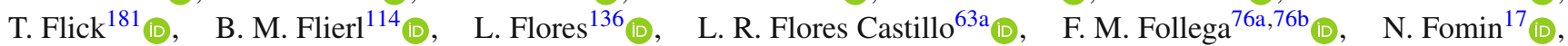

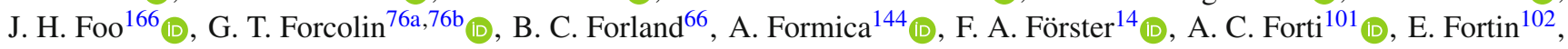

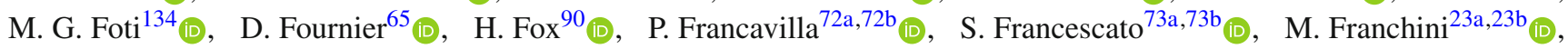

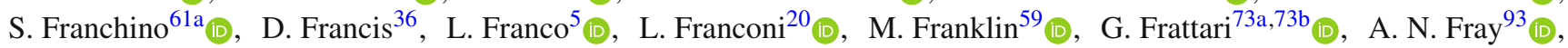

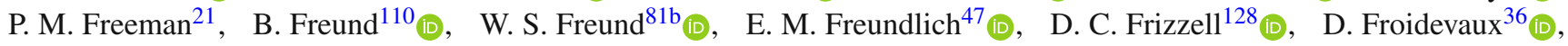

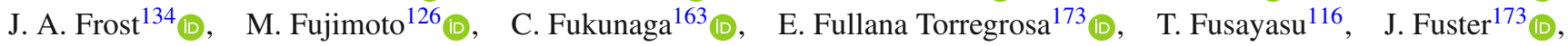

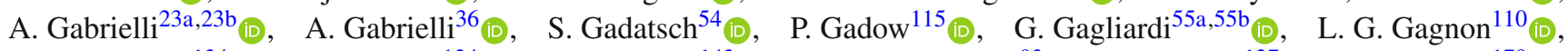
G. E. Gallardo ${ }^{134}$ (D), E. J. Gallas ${ }^{134}$ (I),$\quad$ B. J. Gallop ${ }^{143}$ (D), R. Gamboa Goni ${ }^{33}$ (D), K. K. Gan ${ }^{127}$ (D), S. Ganguly ${ }^{179}$ (D),

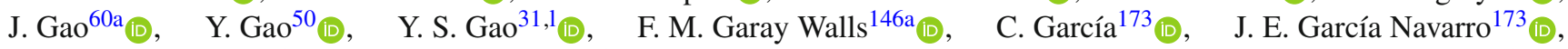

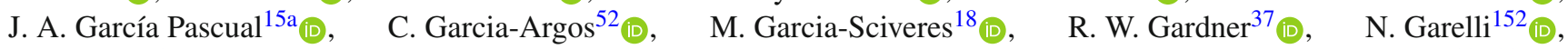
S. Gargiulo ${ }^{52}$ (D), C. A. Garner ${ }^{166}$, V. Garonne ${ }^{133}$ (D), S. J. Gasiorowski ${ }^{147}$ (D), P. Gaspar ${ }^{81 b}$ (D), A. Gaudiello ${ }^{55 a, 55 b}$ (D), $^{2}$,

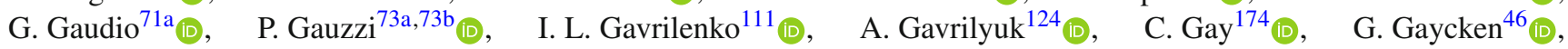

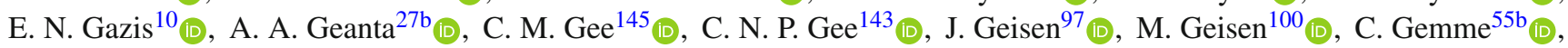

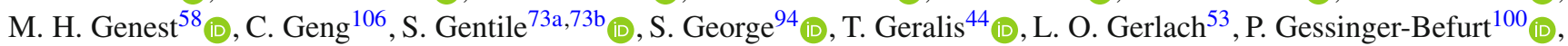
G. Gessner ${ }^{47}$ (1), S. Ghasemi ${ }^{150}$ (D), M. Ghasemi Bostanabad ${ }^{175}$ (D), M. Ghneimat ${ }^{150}$ (D), A. Ghosh ${ }^{65}$ (I), A. Ghosh $^{78}$ (D),

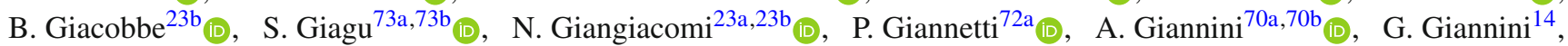

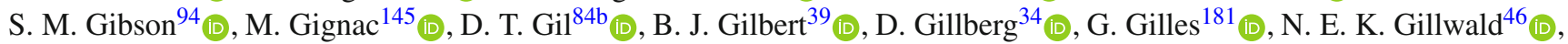

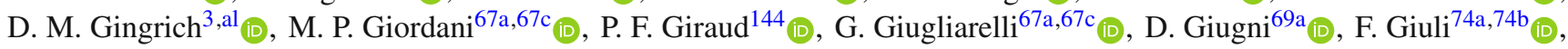

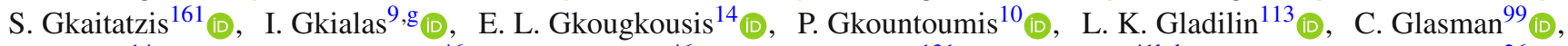

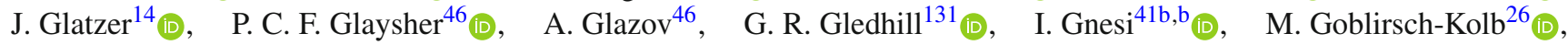

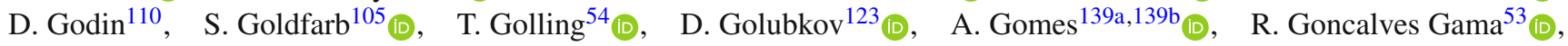

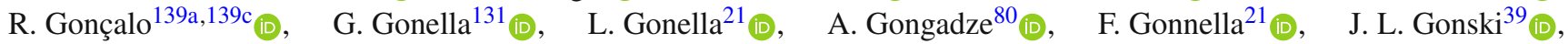
S. González de la Hoz ${ }^{173}$ (D), $\quad$ S. Gonzalez Fernandez ${ }^{14}$ (D) $\quad$ R. Gonzalez Lopez ${ }^{91}$ (D), $\quad$ C. Gonzalez Renteria ${ }^{18}$ (D), R. Gonzalez Suarez ${ }^{171}$ (ID), S. Gonzalez-Sevilla ${ }^{54}$ (D), G. R. Gonzalvo Rodriguez ${ }^{173}$ (I) L. Goossens ${ }^{36}$ (I) N. A. Gorasia ${ }^{21}$,

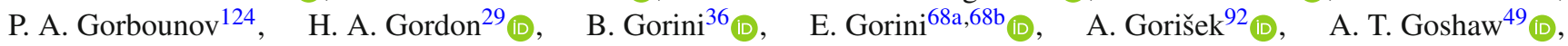

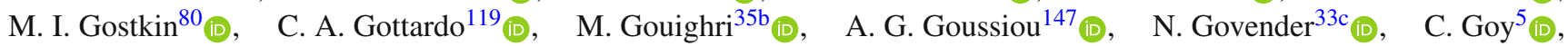
I. Grabowska-Bold ${ }^{84 a}$ (D), E. C. Graham ${ }^{91}$ (D), J. Gramling ${ }^{170}$, E. Gramstad ${ }^{133}$ (I) , S. Grancagnolo ${ }^{19}$ (I), M. Grandi ${ }^{155}$ (D),

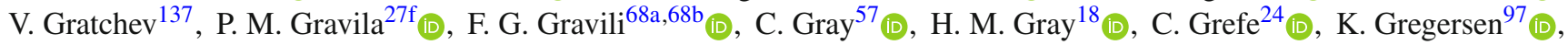

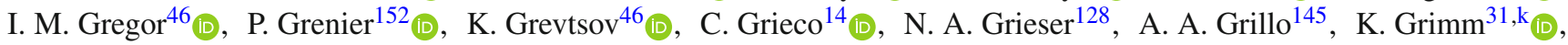

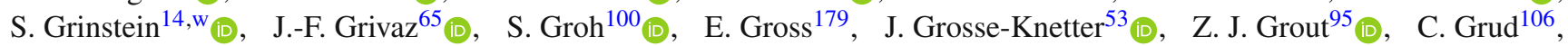




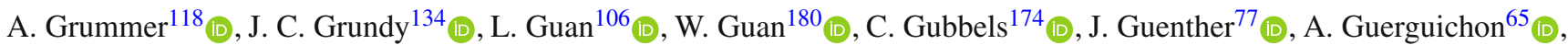
J. G. R. Guerrero Rojas ${ }^{173}$ (D), F. Guescini ${ }^{115}$ (D), D. Guest ${ }^{170}$ (D), R. Gugel ${ }^{100}$ (D), A. Guida ${ }^{46}$ (D), T. Guillemin ${ }^{5}$ (D),

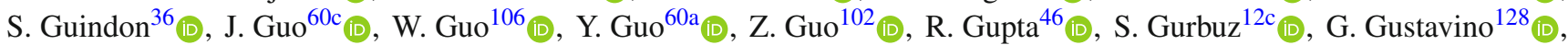
M. Guth ${ }^{52}$ (D) P. Gutierrez ${ }^{128}$ (D) , C. Gutschow ${ }^{95}$ (D) C. Guyot ${ }^{144}$, C. Gwenlan ${ }^{134}$ (D) C. B. Gwilliam ${ }^{91}$ (D) E. S. Haaland ${ }^{133}$ (D),

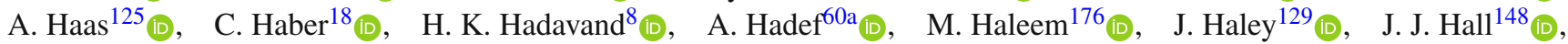

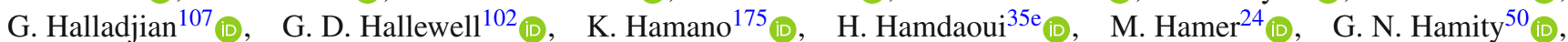

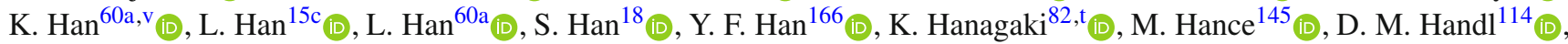

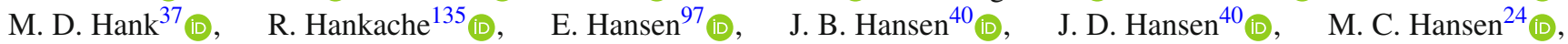
P. H. Hansen ${ }^{40}(\mathbb{D}) \quad$ E. C. Hanson ${ }^{101}(\mathbb{D}), \quad$ K. Hara ${ }^{168}$ (D), T. Harenberg ${ }^{181}$ (D), S. Harkusha ${ }^{108}$ (D), P. F. Harrison ${ }^{177}$, N. M. Hartman ${ }^{152}$ (D), N. M. Hartmann ${ }^{114}$ (D) Y. Hasegawa ${ }^{149}$ (D) A. Hasib ${ }^{50}$ (D) S. Hassani ${ }^{144}$ (D) S. Haug ${ }^{20}$ (D) R. Hauser ${ }^{107}$ (D),

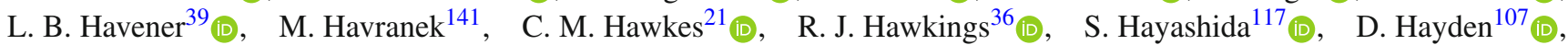
C. Hayes ${ }^{106}$ (D), R. L. Hayes ${ }^{174}$ (D), C. P. Hays ${ }^{134}$ (D), J. M. Hays ${ }^{93}$ (D), H. S. Hayward ${ }^{91}$ (D), S. J. Haywood ${ }^{143}$ (D), F. He Hea $^{60}$, Y. $\mathrm{He}^{164}\left(\mathbb{D}, \quad\right.$ M. P. Heath ${ }^{50}$ (D), V. Hedberg ${ }^{97}$ (D), A. L. Heggelund ${ }^{133}$ (D), C. Heidegger ${ }^{52}$ (D), K. K. $\operatorname{Heidegger}^{52}{ }_{(\mathbb{D})}$,

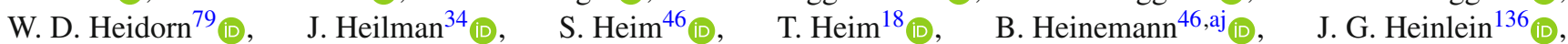

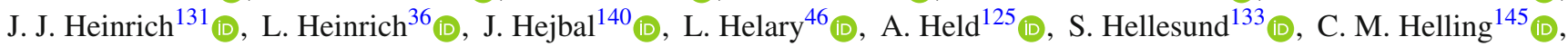
S. Hellman ${ }^{45 a, 45 b}$ (D), C. Helsens ${ }^{36}{ }_{(\mathbb{D})}$, R. C. W. Henderson ${ }^{90}$, Y. Heng ${ }^{180}$, L. Henkelmann ${ }^{32}$ (D), A. M. Henriques Correia ${ }^{36}$, H. Herde ${ }^{26}$ (D), Y. Hernández Jiménez ${ }^{33} e_{(D)}, \quad$ H. Herr ${ }^{100}, \quad$ M. G. Herrmann ${ }^{114}$ (D), T. Herrmann ${ }^{48}$ (D), G. Herten ${ }^{52}$ (D),

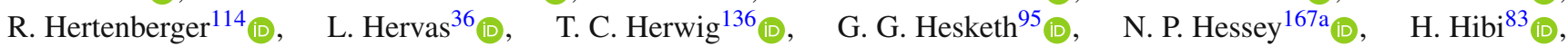
S. Higashino ${ }^{82}$ (D) , E. Higón-Rodriguez ${ }^{173}$ (D), K. Hildebrand ${ }^{37}$, J. C. Hill ${ }^{32}$ (D) , K. K. Hill ${ }^{29}$ (D) , K. H. Hiller ${ }^{46}$, S. J. Hillier ${ }^{21}$ (D),

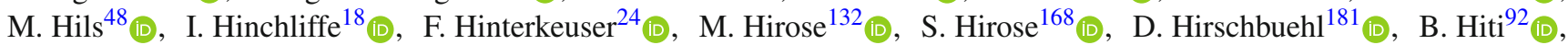

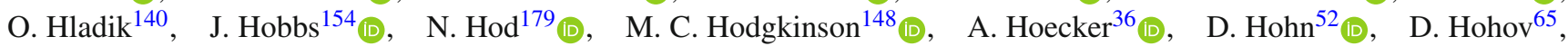
T. Holm ${ }^{24}$ (D), T. R. Holmes ${ }^{37}$ (D), M. Holzbock ${ }^{115}$ (D), L. B. A. H. Hommels ${ }^{32}$ (D), T. M. Hong ${ }^{138}$ (D), J. C. Honig ${ }^{52}$ (D), A. Hönle ${ }^{115}$ (D), B. H. Hooberman ${ }^{172}$ (D), W. H. Hopkins ${ }^{6}$ (D) Y. Horii ${ }^{117}$ (D), P. Horn ${ }^{48}$ (D) L. A. Horyn ${ }^{37}$ (D), S. Hou ${ }^{157}$ (D), A. Hoummada ${ }^{35 a}$, J. Howarth ${ }^{57}$ (D), J. Hoya ${ }^{89}$ (D), M. Hrabovsky ${ }^{130}$ (D), J. Hrivnac ${ }^{65}$, A. Hrynevich ${ }^{109}$ (D), T. Hryn'ova ${ }^{5}$ (D),

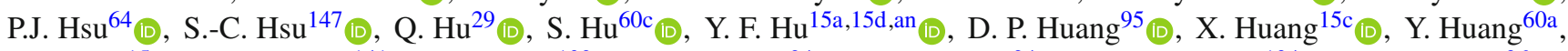

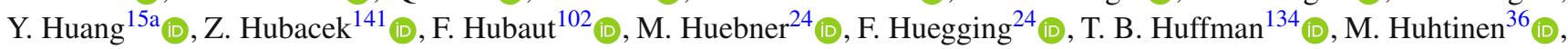

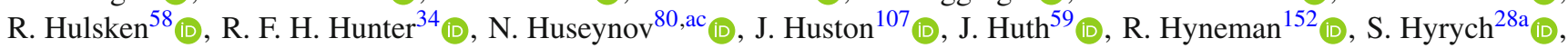

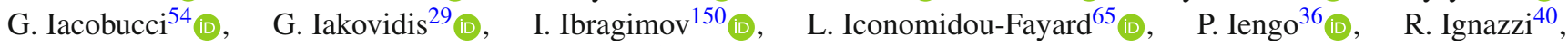

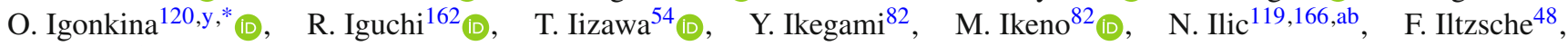

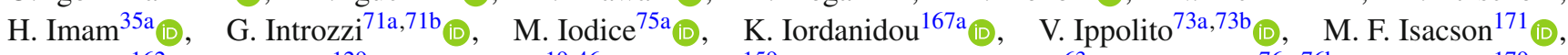

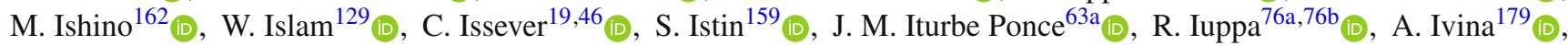
J. M. Izen ${ }^{43}$ (I) V. Izzo $^{70 a}$ (D), P. Jacka ${ }^{140}$ (D), P. Jackson ${ }^{1}$ (D), R. M. Jacobs ${ }^{46}$ (D) B. P. Jaeger ${ }^{151}$ (D), V. Jain ${ }^{2}$ (D), G. Jäkel ${ }^{181}$ (D), K. B. Jakobi ${ }^{100}$, K. Jakobs ${ }^{52}$ (D), T. Jakoubek ${ }^{179}$ (D), J. Jamieson ${ }^{57}$ (D) , K. W. Janas ${ }^{84 a}$ (D), R. Jansky ${ }^{54}$ (I), M. Janus ${ }^{53}$ (D),

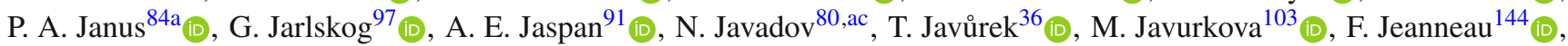

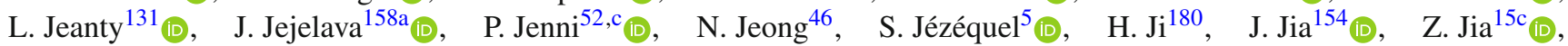

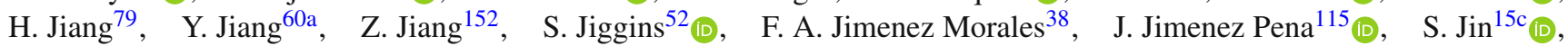

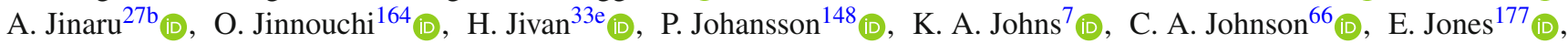

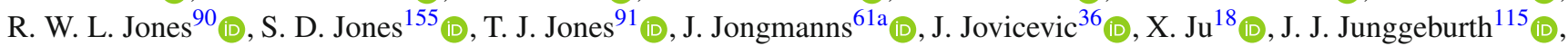

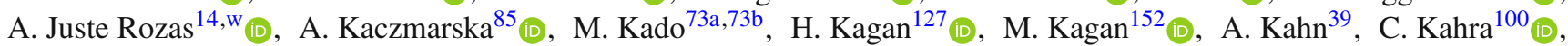
T. $\mathrm{Kaji}^{178}$ (D), E. Kajomovitz ${ }^{159}$ (D), C. W. Kalderon ${ }^{29}$ (D), A. Kaluza ${ }^{100}, \quad$ A. Kamenshchikov ${ }^{123}$ (D), M. Kaneda ${ }^{162}$ (D),

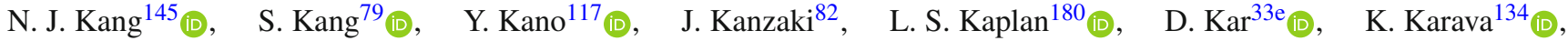

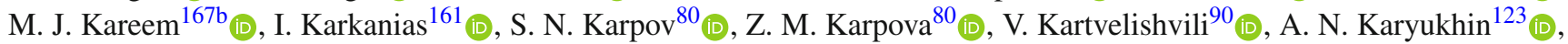

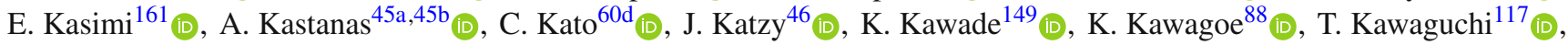

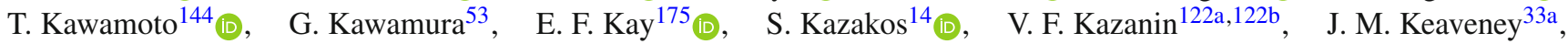
R. Keeler ${ }^{175}$ (D) J. S. Keller ${ }^{34}$ (D), E. Kellermann ${ }^{97}$, D. Kelsey ${ }^{155}$ (D) J. J. Kempster ${ }^{21}$ (D), J. Kendrick ${ }^{21}$ (D) K. E. Kennedy ${ }^{39}$ (D), O. Kepka ${ }^{140}$, S. Kersten ${ }^{181}$, B. P. Kerševan ${ }^{92}$ (D), S. Ketabchi Haghighat ${ }^{166}$ (D), M. Khader ${ }^{172}$ (D), F. Khalil-Zada ${ }^{13}$,

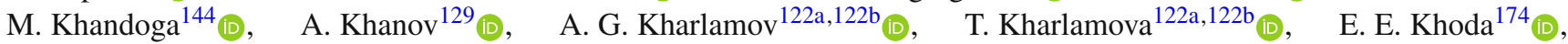
A. Khodinov ${ }^{165}$ (D), T. J. Khoo $^{77}$ (D), G. Khoriauli ${ }^{176}$ (D), E. Khramov ${ }^{80}$ (D) J. Khubua ${ }^{158 b}$ (D), S. Kido ${ }^{83}$ (D) M. Kiehn ${ }^{36}$ (D), E. $\operatorname{Kim}^{164}$ (D), Y. K. $\operatorname{Kim}^{37}$ (D), N. Kimura ${ }^{95}$, A. Kirchhoff ${ }^{53}$ (D), D. $\operatorname{Kirchmeier}^{48}$ (D), J. Kirk ${ }^{143}$ (D), A. E. Kiryunin ${ }^{115}$ (D), T. Kishimoto ${ }^{162}$ (D), D. P. Kisliuk ${ }^{166}$, V. Kitali ${ }^{46}$ (D), C. Kitsaki ${ }^{10}$ (D) O. Kivernyk ${ }^{24}$ (D), T. Klapdor-Kleingrothaus ${ }^{52}$ (D), M. Klassen ${ }^{61 \mathrm{a}}$ (D), C. Klein ${ }^{34}$, M. H. Klein ${ }^{106}$ (D), M. Klein ${ }^{91}$ (D), U. Klein ${ }^{91}$ (D), K. Kleinknecht ${ }^{100}$, P. Klimek ${ }^{36}$ (D),

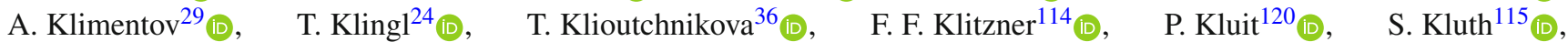

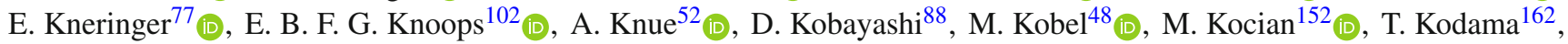




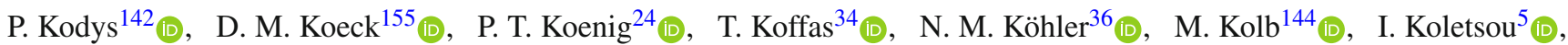
T. Komarek ${ }^{130}$ (D), T. Kondo ${ }^{82}$, K. Köneke ${ }^{52}$ (D), A. X. Y. Kong ${ }^{1}$ (D), A. C. König ${ }^{119}$ (D), T. Kono ${ }^{126}$ (D), V. Konstantinides ${ }^{95}$,

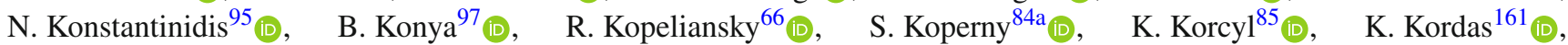
G. Koren ${ }^{160}$, A. $K \operatorname{ron}^{95}$ (D) I. Korolkov ${ }^{14}$ (D), E. V. Korolkova ${ }^{148}$, N. Korotkova ${ }^{113}$ (D), O. Kortner ${ }^{115}$ (D), S. Kortner ${ }^{115}$ (D), V. V. Kostyukhin ${ }^{148,165}$ (D) A. Kotsokechagia ${ }^{65}$ (D) A. Kotwal $^{49}$ (D), A. Koulouris ${ }^{10}$ (D), A. Kourkoumeli-Charalampidi ${ }^{71 a}, 71 b$ (D),

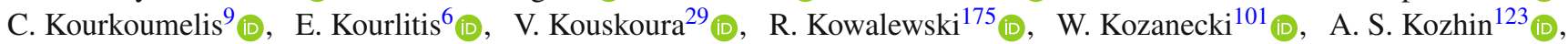

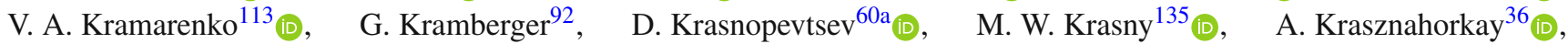

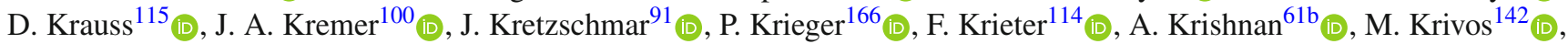
K. Krizka ${ }^{18}$ (D) K. Kroeninger ${ }^{47}$ (D) H. Kroha ${ }^{115}$ (D) J. Kroll ${ }^{140}$ (D) J. Kroll ${ }^{136}$ (D) K. S. Krowpman ${ }^{107}$ (D), U. Kruchonak ${ }^{80}$ (D), H. Krüger ${ }^{24}$ (D), N. Krumnack ${ }^{79}$, M. C. Kruse $^{49}$ (D), J. A. Krzysiak ${ }^{85}$ (D) A. Kubota ${ }^{164}$ (D), O. Kuchinskaia ${ }^{165}$, S. Kuday ${ }^{4 b}$ (D),

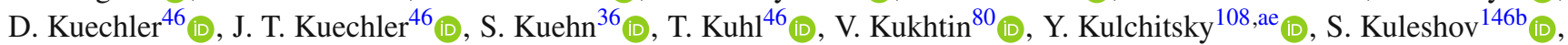

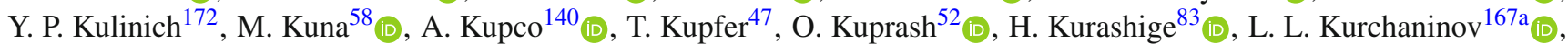

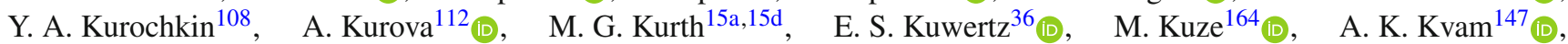

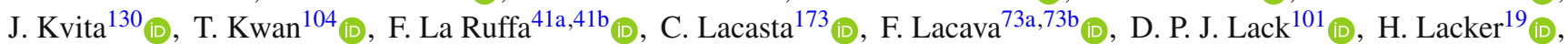

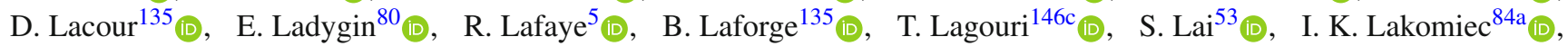

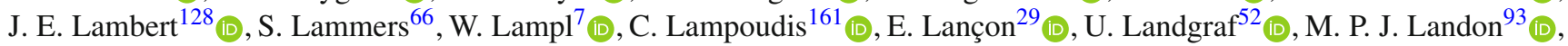

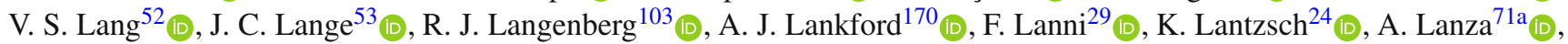

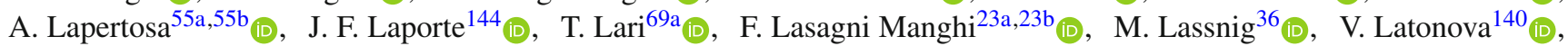

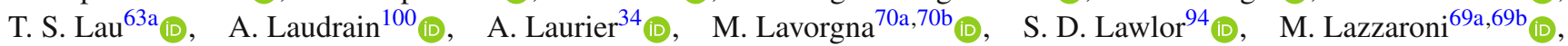
B. Le ${ }^{101}$, E. Le Guirriec ${ }^{102}$ (D) A. Lebedev ${ }^{79}$ (D) M. LeBlanc ${ }^{7}$ (D) T. LeCompte ${ }^{6}$ (D) F. Ledroit-Guillon ${ }^{58}$ (ID, A. C. A. Lee ${ }^{95}$,

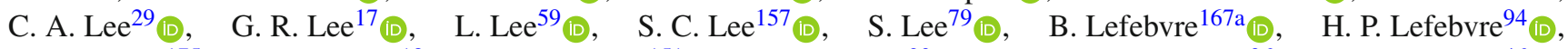

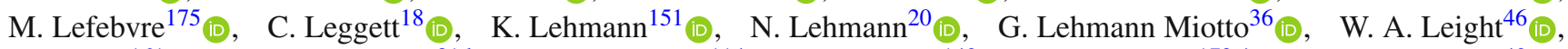
A. Leisos ${ }^{161, \mathrm{u}}$ (D), M. A. L. Leite ${ }^{81 d}$ (D), C. E. Leitgeb ${ }^{114}$ (D), R. Leitner ${ }^{142}$ (D), D. Lellouch ${ }^{179, *}$ (D), K. J. C. Leney ${ }^{42}$ (D),

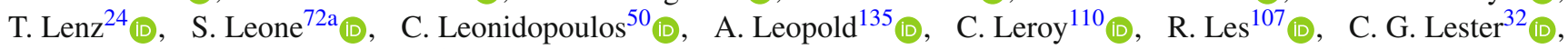

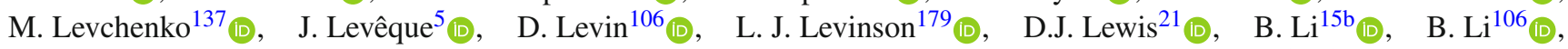

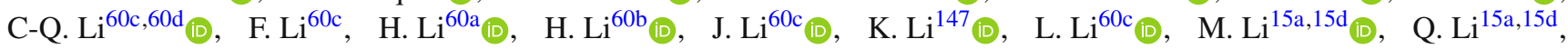

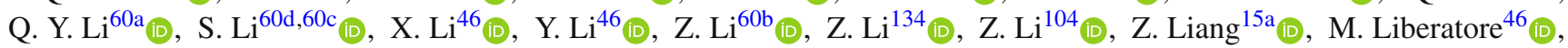

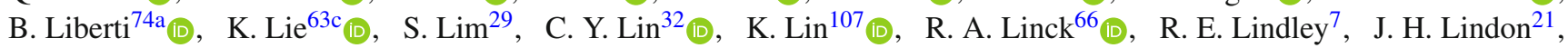

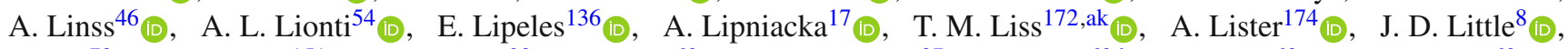

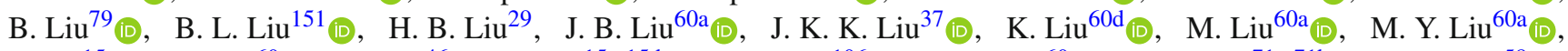

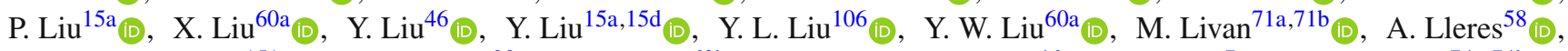

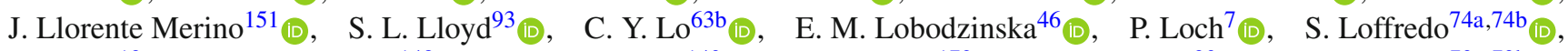

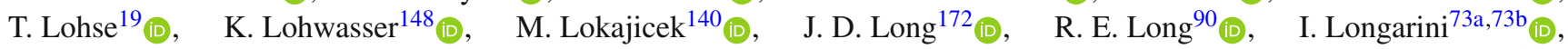
L. Longo ${ }^{36}$ (1), K. A. Looper ${ }^{127}$ (D), I. Lopez Paz ${ }^{101}$, A. Lopez Solis ${ }^{148}$ (D), J. Lorenz ${ }^{114}$ (I), N. Lorenzo Martinez ${ }^{5}$ (D),

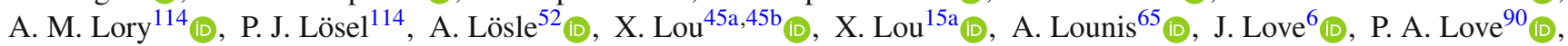

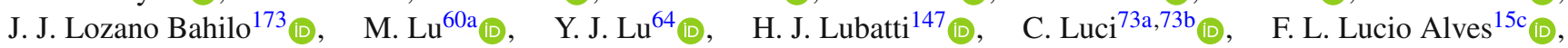
A. Lucotte ${ }^{58}$ (D), F. Luehring ${ }^{66}$ (D), I. Luise ${ }^{154}$ (D), L. Luminari ${ }^{73 a}$, B. Lund-Jensen ${ }^{153}$ (D), M. S. Lutz ${ }^{160}$ (D), D. Lynn ${ }^{29}$ (D),

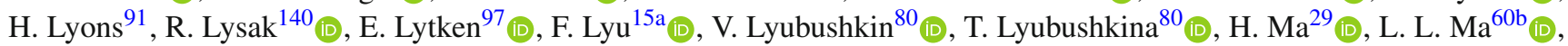
Y. Ma ${ }^{95}$ (D) D. M. Mac Donell ${ }^{175}$ (D) , G. Maccarrone ${ }^{51}$ (D), A. Macchiolo ${ }^{115}$ (D) C. M. Macdonald ${ }^{148}$ (D) J. C. MacDonald ${ }^{148}$ (D),

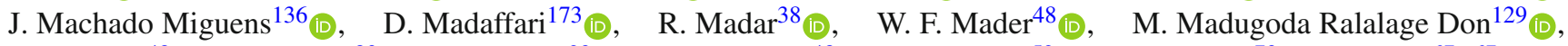

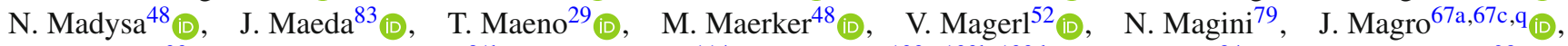

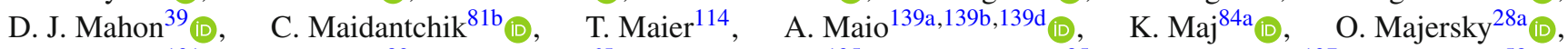
S. Majewski ${ }^{131}$ (D), Y. Makida ${ }^{82}$, N. Makovec $^{65}$ (D), B. Malaescu ${ }^{135}$ (D) Pa. Malecki ${ }^{85}$ (I), V. P. Maleev ${ }^{137}$ (D), F. Malek ${ }^{58}$ (D),

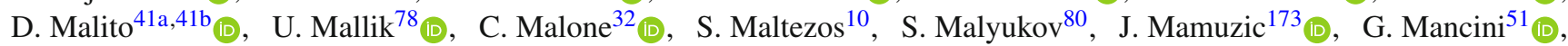
I. Mandić92 (D) L. Manhaes de Andrade Filho ${ }^{81 a}$ (D) I. M. Maniatis ${ }^{161}$ (D) J. Manjarres $\operatorname{Ramos}^{48}$ (D) K. H. Mankinen ${ }^{97}$ (D),

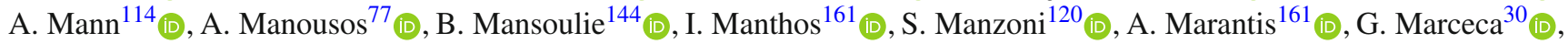

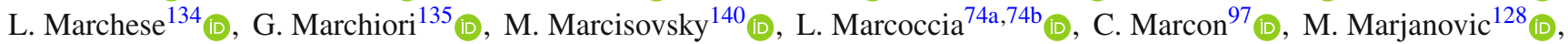

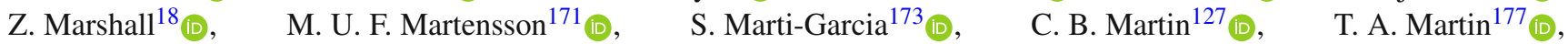

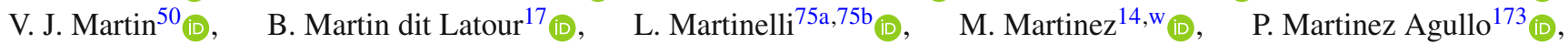

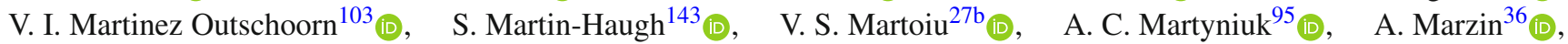
S. R. Maschek ${ }^{115}$ (D) L. Masetti ${ }^{100}$ (D), T. Mashimo ${ }^{162}$ (D) , R. Mashinistov ${ }^{111}$ (D) J. Masik ${ }^{101}$ (D), A. L. Maslennikov ${ }^{122 a, 122 b}$ (D),

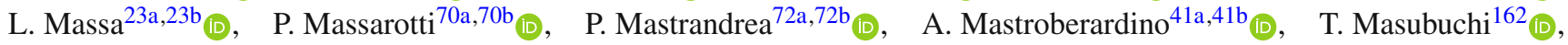

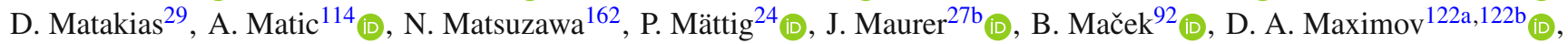




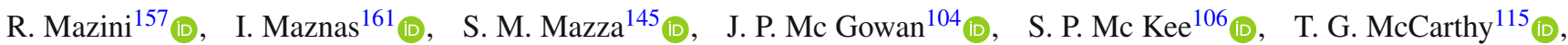

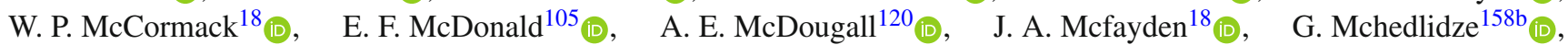

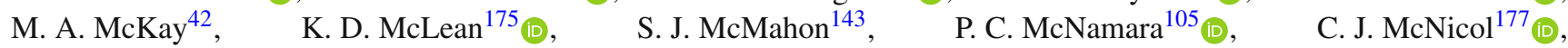

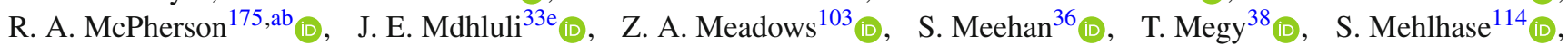

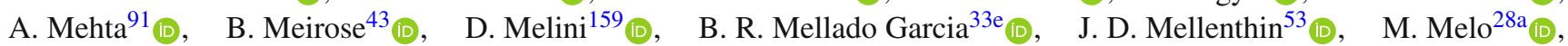

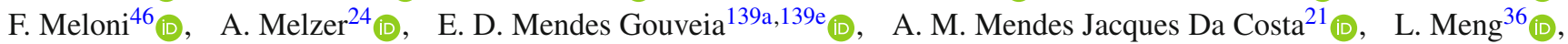
X. T. Meng ${ }^{106}$ (ID), S. Menke ${ }^{115}$ (D), $\quad$ E. Meoni ${ }^{41 \mathrm{a}, 41 \mathrm{~b}}, \quad$ S. Mergelmeyer ${ }^{19}, \quad$ S. A. M. Merkt ${ }^{138}, \quad$ C. Merlassino ${ }^{134} \mathbb{C}_{(\mathrm{D})}$,

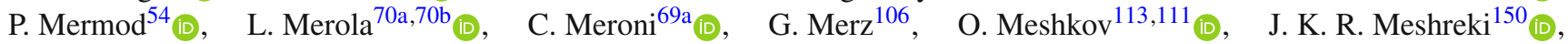

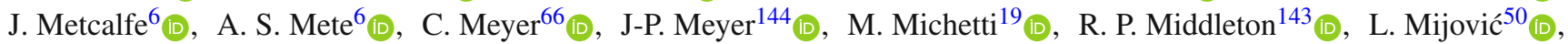

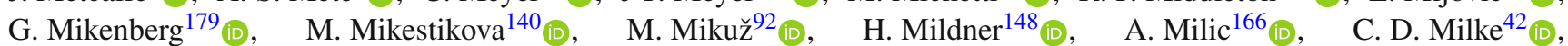
D. W. Mille ${ }^{37}$ (D), L. S. Miller ${ }^{34}$ (D, A. Milov ${ }^{179}$ (D), D. A. Milstead ${ }^{45 a, 45 b}, \quad$ R. A. Mina ${ }^{152}$ (D), A. A. Minaenko ${ }^{123}$ (D), I. A. Minashvili ${ }^{158 b}$ (D), L. Mince ${ }^{57}$ (D), A. I. Mincer $^{125}$ (D), B. Mindur ${ }^{84 a}$ (D), M. Mineev ${ }^{80}$ (D), Y. Minegishi ${ }^{162}$, Y. Mino ${ }^{86}$ (D),

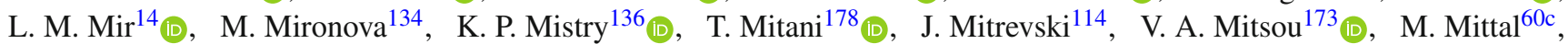

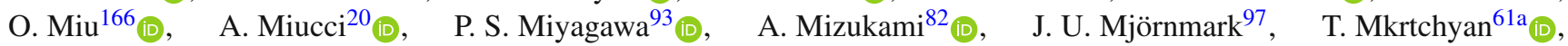

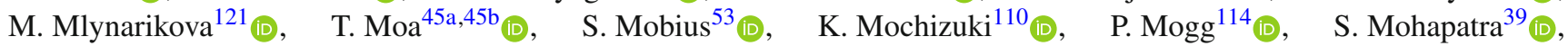
R. Moles-Valls ${ }^{24}$ (D) K. Mönig ${ }^{46}$ (D), E. Monnier ${ }^{102}$ (D), A. Montalbano ${ }^{151}$ (D), J. Montejo Berlingen ${ }^{36}$ (D) M. Montella ${ }^{95}$ (D),

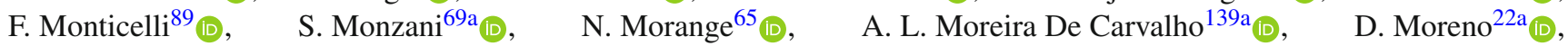
M. Moreno Llácer ${ }^{173}$ (D), C. Moreno Martinez ${ }^{14}$ (i),$\quad$ P. Morettini ${ }^{55 b}$ (D), M. Morgenstern ${ }^{159}$ (i), S. Morgenstern ${ }^{48}$ (D),

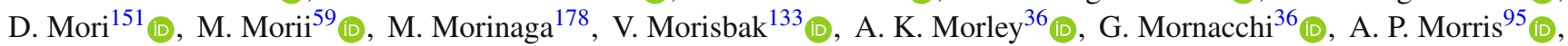

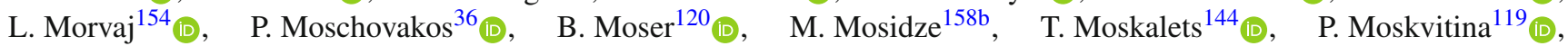
J. Moss ${ }^{31, m_{1}}$, E. J. W. Moyse ${ }^{103}$ (D), S. Muanza ${ }^{102}$ (D), J. Mueller ${ }^{138}$ (D), R. S. P. Mueller ${ }^{14}$, D. Muenstermann ${ }^{90} \mathbb{C}_{\text {D }}$,

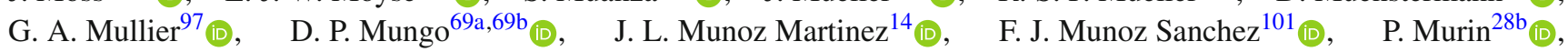

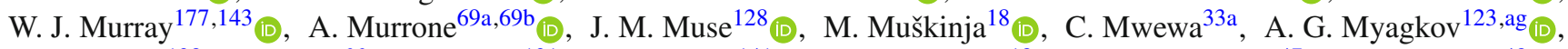
A. A. Myers ${ }^{138}$, G. Myers ${ }^{66}$ (D) J. Myers ${ }^{131}$ (D) M. Myska ${ }^{141}$ (D), B. P. Nachman ${ }^{18}$ (D), O. Nackenhorst ${ }^{47}$ (D) A. Nag Nag $^{48}$ (D), K. Nagai ${ }^{134}$ (D), K. Nagano ${ }^{82}$ (D), Y. Nagasaka ${ }^{62}$ (D) J. L. Nagle ${ }^{29}$ (D), E. Nagy ${ }^{102}$ (D), A. M. Nairz ${ }^{36}$ (D), Y. Nakahama ${ }^{117}$ (D), K. Nakamura ${ }^{82}$ (D), T. Nakamura ${ }^{162}$ (D), H. Nanjo ${ }^{132}$ (D), F. Napolitano ${ }^{61 a^{(D)}, \text { R. F. Naranjo Garcia }}{ }^{46}$ (D), R. Narayan ${ }^{42}$ (D),

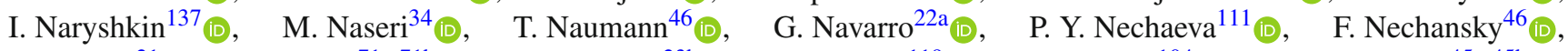

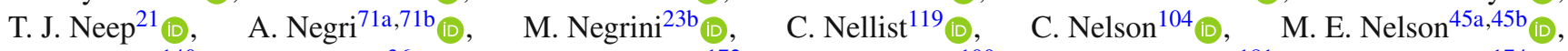

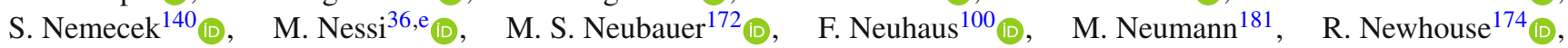

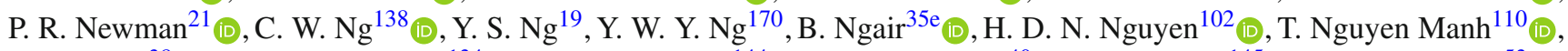
E. Nibigira ${ }^{38}$ (D), R. B. Nickerson ${ }^{134}$ (D), R. Nicolaidou ${ }^{144}$ (D), D. S. Nielsen ${ }^{40}$ (D), J. Nielsen ${ }^{145}$ (D), M. Niemeyer ${ }^{53}$ (D), N. Nikiforou ${ }^{11}$ (D), V. Nikolaenko ${ }^{123, a^{(D)}}$, I. Nikolic-Audit ${ }^{135}$ (D), K. Nikolopoulos ${ }^{21}$ (D) P. Nilsson ${ }^{29}$ (D) H. R. Nindhito ${ }^{54}$ (D),

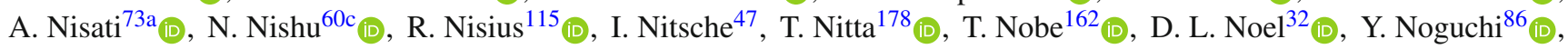

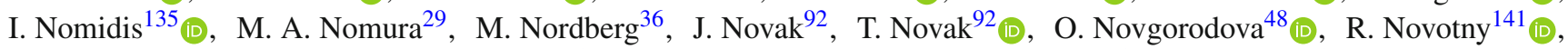

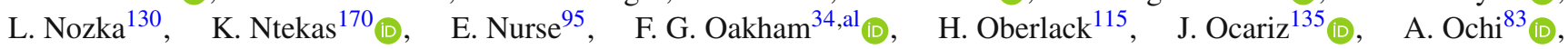

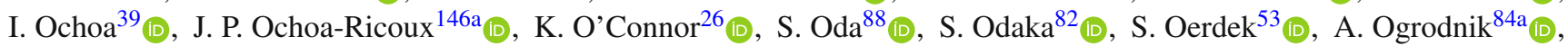

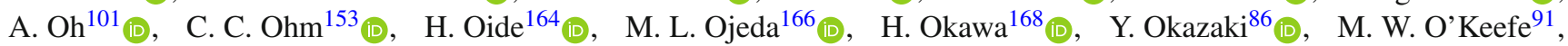

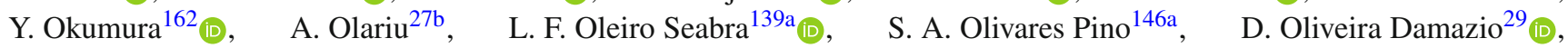
J. L. Oliver ${ }^{1}$ (D), M. J. R. Olsson ${ }^{170}$ (D), A. Olszewski ${ }^{85}$ (D), J. Olszowska ${ }^{85}$ (D), Ö. O. Öncel ${ }^{24}$ (D), D. C. O’Neil ${ }^{151}$ (D), A. P. O'neill ${ }^{134}$ (D) A. Onofre ${ }^{139 a, 139 e}$ (D) P. U. E. Onyisi ${ }^{11}$ (D) H. Oppen ${ }^{133}$, R. G. Oreamuno Madriz ${ }^{121}$, M. J. Oreglia ${ }^{37}$ (D),

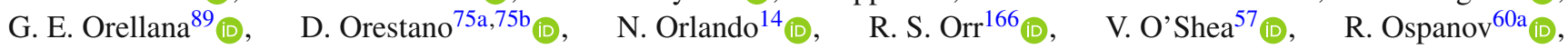

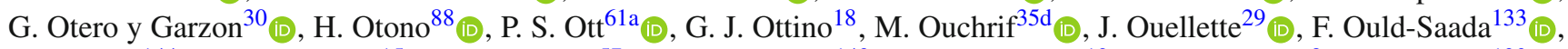

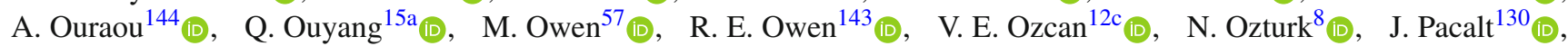

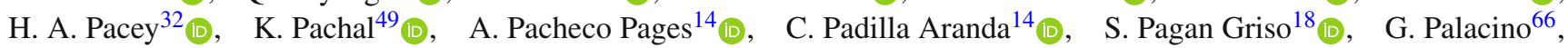
S. Palazzo ${ }^{50}$, S. Palestini ${ }^{36}$ (I) , M. Palka ${ }^{84 b}$ (D), P. Palni ${ }^{84 a}$ (D), C. E. Pandini ${ }^{54}$ (D) J. G. Panduro Vazquez ${ }^{94}$ (D), P. Pani ${ }^{46}$ (D),

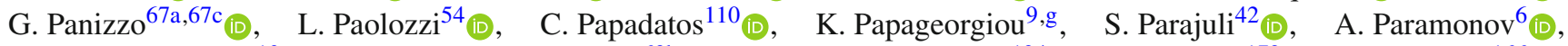

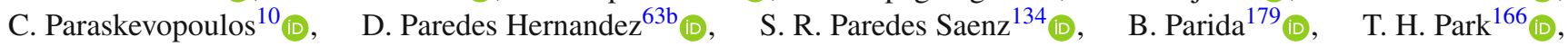

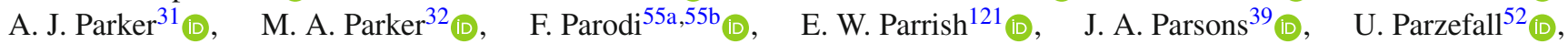
L. Pascual Dominguez ${ }^{135}$ (D), V. R. Pascuzzi ${ }^{18}$ (D), J. M. P. Pasner ${ }^{145}$ (D), F. Pasquali ${ }^{120}$ (D), $\quad$ E. Pasqualucci $^{73 a^{\infty} \text {, }}$,

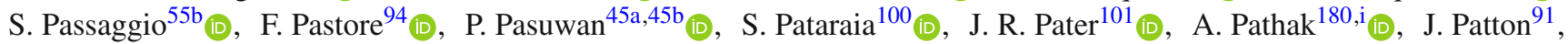

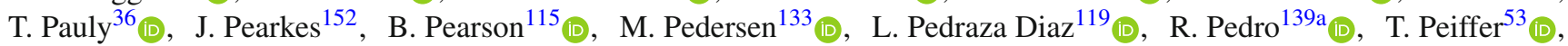

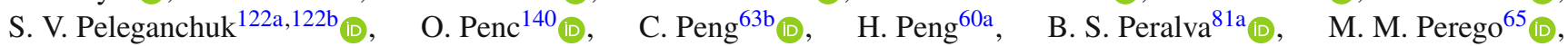

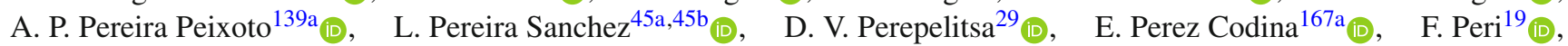




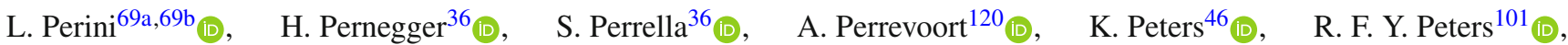

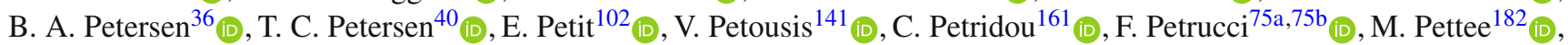

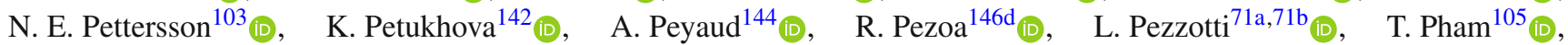

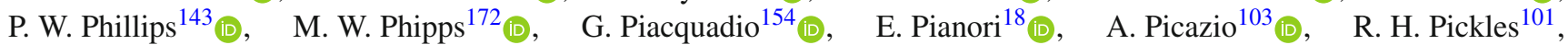

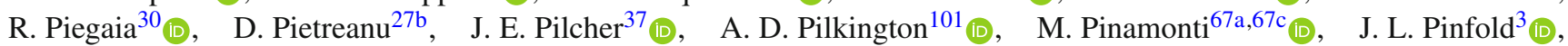

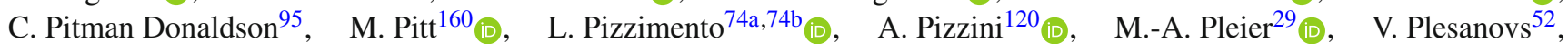
V. Pleskot ${ }^{142}$ (D), E. Plotnikova ${ }^{80}, \quad$ P. Podberezko ${ }^{122 a, 122 b}$ (D), R. Poettgen ${ }^{97}$ (D), R. Poggi ${ }^{54}$ (D), L. Poggioli $^{135}$ (D),

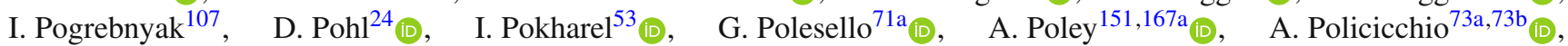
R. Polifka ${ }^{142}$ (D), A. Polini ${ }^{23 b}$ (D) $_{\text {, C. S. Pollard }}^{46}{ }_{(\text {D }}$, V. Polychronakos ${ }^{29}$ (D), D. Ponomarenko ${ }^{112}$ (D), L. Pontecorvo ${ }^{36}$ (D),

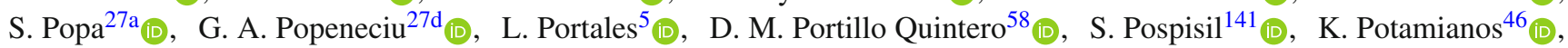
I. N. Potrap ${ }^{80}$ (i), C. J. Potter ${ }^{32}$ (D), H. Potti ${ }^{11}$ (D), T. Poulsen ${ }^{97}$ (D), J. Poveda ${ }^{173}$ (D), T. D. Powell ${ }^{148}$ (D), G. Pownall ${ }^{46}$, M. E. Pozo Astigarraga ${ }^{36}$ (D) A. Prades Ibanez ${ }^{173}$ (D), P. Pralavorio ${ }^{102}$ (D), M. M. Prapa ${ }^{44}$ (D), S. Prell ${ }^{79}$ (D), D. Price ${ }^{101}$ (D),

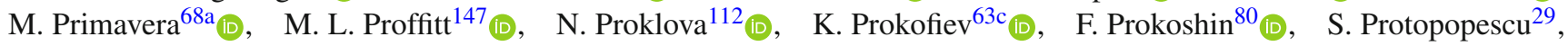
J. Proudfoot ${ }^{6}$ (D) M. Przybycien ${ }^{84 a}$ (D), D. Pudzha ${ }^{137}$ (D), A. Puri ${ }^{172}$ (D), P. Puzo ${ }^{65}$, D. Pyatiizbyantseva ${ }^{112}$ (D), J. Qian ${ }^{106}$ (D),

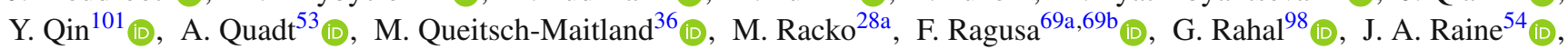

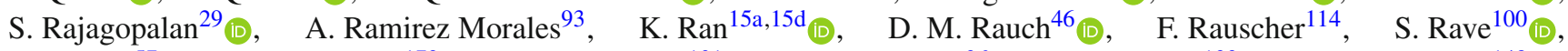

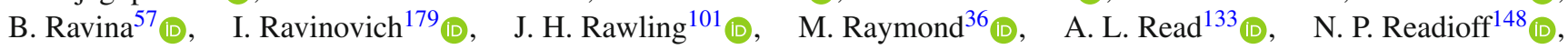

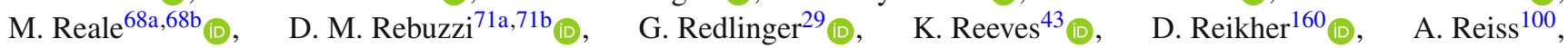

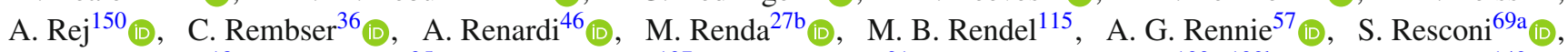

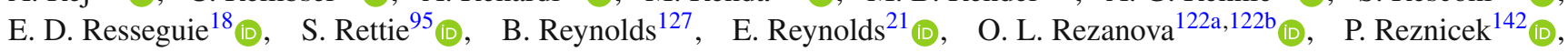

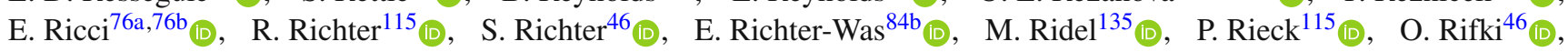

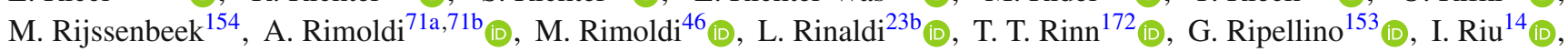

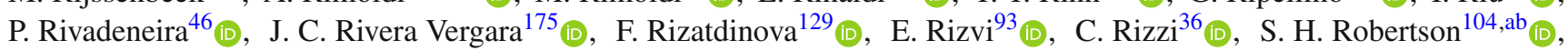

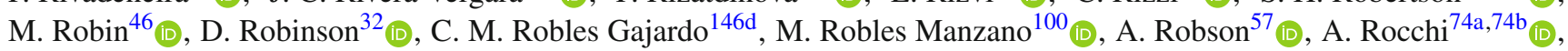

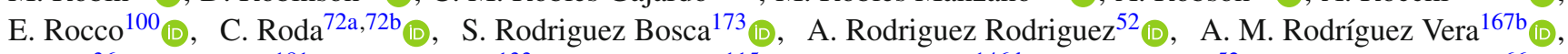

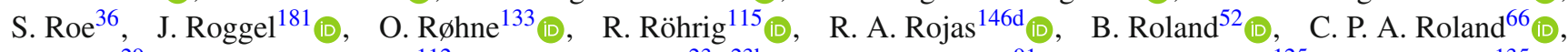

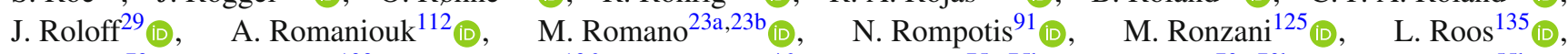

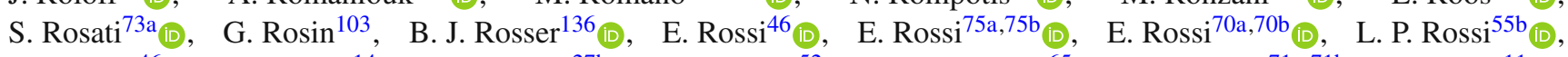

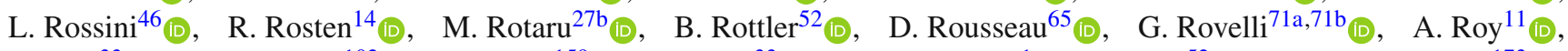

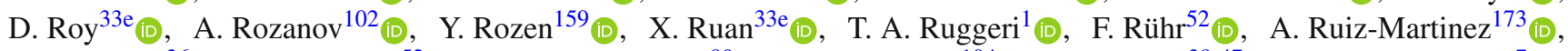
A. Rummler ${ }^{36}$ (D), Z. Rurikova ${ }^{52}$ (D), N. A. Rusakovich ${ }^{80}$ (D) H. L. Russell ${ }^{104}$ (I) , L. Rustige ${ }^{38,47}$ (D), J. P. Rutherfoord ${ }^{7}$, E. M. Rüttinger ${ }^{148}$ (D), M. Rybar ${ }^{142}, \quad$ G. Rybkin ${ }^{65}$ (D), E. B. Rye ${ }^{133}$ (D), A. Ryzhov ${ }^{123}$ (D), J. A. Sabater Iglesias ${ }^{46}$ (D),

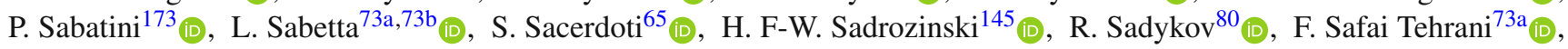

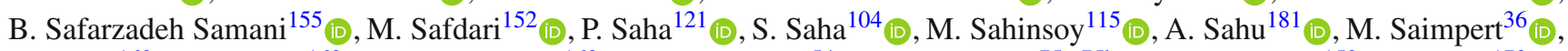

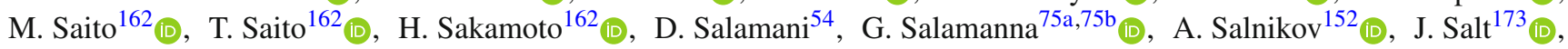

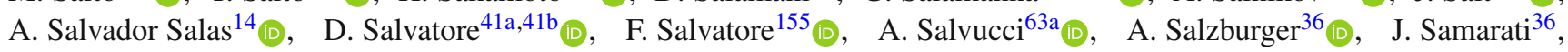

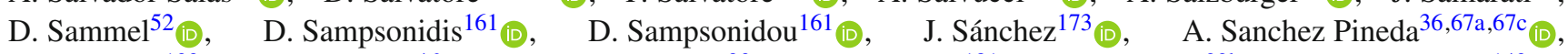

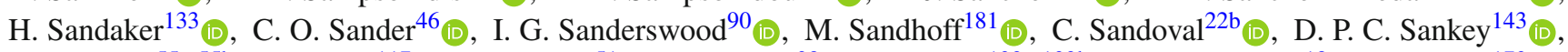

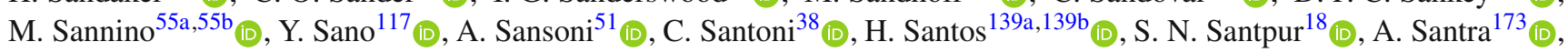

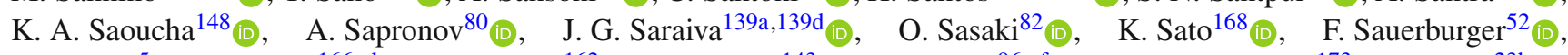

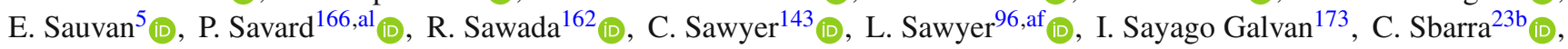
A. Sbrizzi ${ }^{67 a}, 67 \mathrm{c}\left(\mathbb{D}, \quad\right.$ T. Scanlon ${ }^{95}$ (D), J. Schaarschmidt ${ }^{147}$ (D), P. Schacht ${ }^{115}$ (D), D. Schaefer ${ }^{37}$ (D), L. Schaefer ${ }^{136} \mathbb{D}_{0}$, U. Schäfer ${ }^{100}$ (D), A. C. Schaffer ${ }^{65}$ (D), D. Schaile ${ }^{114}$ (D), R. D. Schamberger ${ }^{154}$ (D), E. Schanet ${ }^{114}$ (D), C. Scharf ${ }^{19}$ (D),

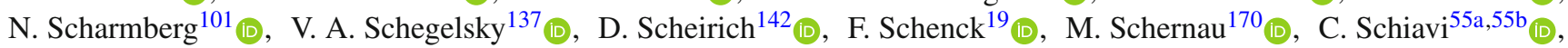

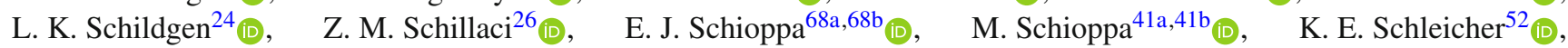

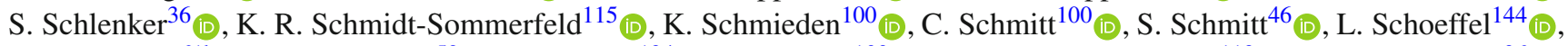

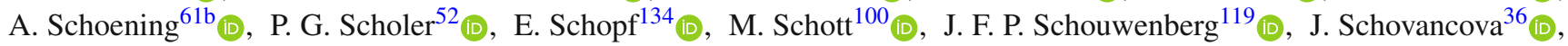
S. Schramm ${ }^{54}$ (D) F. Schroeder ${ }^{181}$ (D), A. Schulte ${ }^{100}$ (D) H-C. Schultz-Coulon ${ }^{61 \mathrm{a}}$ (D) M. Schumacher ${ }^{52}$ (D) B. A. Schumm ${ }^{145}$ (D), Ph. Schune ${ }^{144}$ (D), A. Schwartzman ${ }^{152}$ (D), T. A. Schwarz ${ }^{106}$ (D), Ph. Schwemling ${ }^{144}$ (D), R. Schwienhorst ${ }^{107}$ (D),

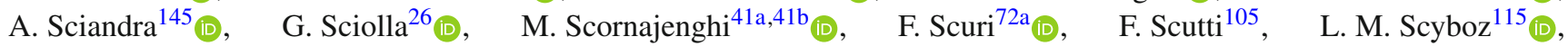
C. D. Sebastiani ${ }^{11}$ (D) K. Sedlaczek ${ }^{47}$ (D) P. Seema ${ }^{19}$ (D) S. C. Seidel ${ }^{118}$ (D), A. Seiden ${ }^{145}$ (D), B. D. Seidlitz ${ }^{29}$ (D), T. Seiss ${ }^{37}$ (D),

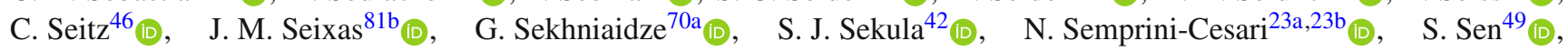

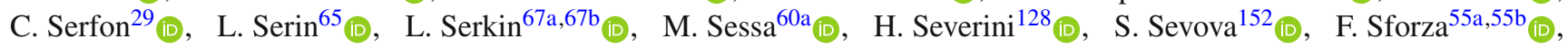




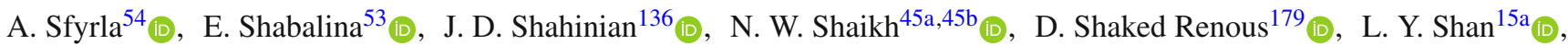
M. Shapiro ${ }^{18}$ (D) A. Sharma ${ }^{36}$ (D) A. S. Sharma ${ }^{1}$ (D), P. B. Shatalov ${ }^{124}$ (D), K. Shaw ${ }^{155}$ (D), S. M. Shaw ${ }^{101}$ (D), M. Shehade ${ }^{179}$, Y. Shen ${ }^{128}$, A. D. Sherman ${ }^{25}$, P. Sherwood ${ }^{95}$ (D) L. Shi ${ }^{95}$ (D), C. O. Shimmin ${ }^{182}$ (D), Y. Shimogama ${ }^{178}$, M. Shimojima ${ }^{116}$ (D), J. D. Shinner ${ }^{94}$ (D), I. P. J. Shipsey ${ }^{134}$ (D), S. Shirabe ${ }^{164}$ (D) $\quad$ M. Shiyakova ${ }^{80, z}$ (D), J. Shlomi ${ }^{179}$ (D), A. Shmeleva ${ }^{111}$, M. J. Shochet ${ }^{37}$ (D) J. Shojaii ${ }^{105}$ (D), D. R. Shope ${ }^{153}$, S. Shrestha ${ }^{127}$ (D), E. M. Shrif ${ }^{33}{ }_{(D)}$, M. J. Shroff ${ }^{175}$ (D), E. Shulga ${ }^{179}{ }_{(\mathbb{D})}$,

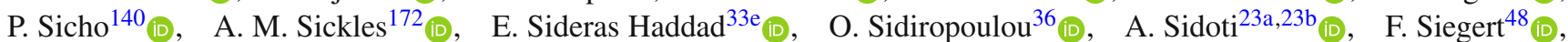

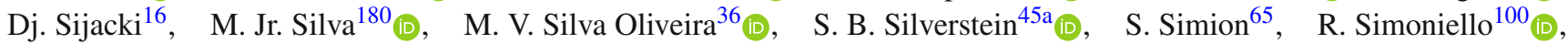

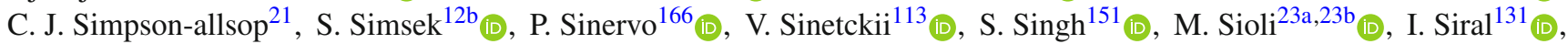
S. Yu. Sivoklokov ${ }^{113}$ (D), J. Sjölin ${ }^{45 a, 45 b}$ (D), A. Skaf ${ }^{53}$ (D), E. Skorda ${ }^{97}$, P. Skubic ${ }^{128}$ (D), M. Slawinska ${ }^{85}$ (D), K. Sliwa ${ }^{169}$ (D),

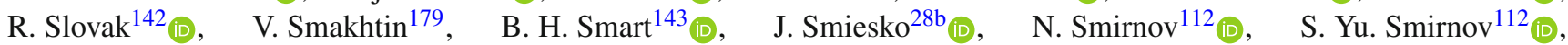

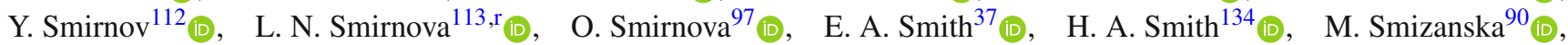
K. Smolek ${ }^{141}$ (D)， A. Smykiewicz ${ }^{85}$ (D), A. A. Snesarev ${ }^{111}$ (D), H. L. Snoek ${ }^{120}$ (D), I. M. Snyder ${ }^{131}$ (D), S. Snyder ${ }^{29}$ (D),

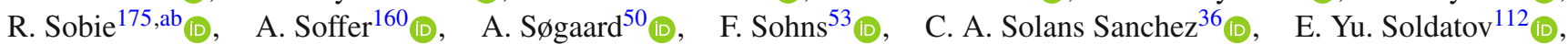
U. Soldevila ${ }^{173}$ (D), A. A. Solodkov ${ }^{123}$ (I) , A. Soloshenko ${ }^{80}$ (D) O. V. Solovyanov ${ }^{123}$ (D), V. Solovyev ${ }^{137}$ (D), P. Sommer ${ }^{148}$ (D),

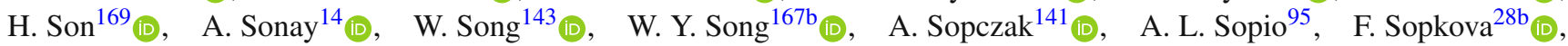

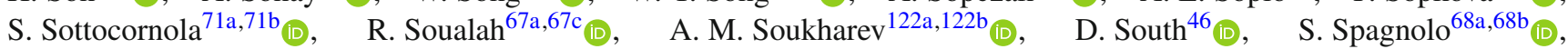

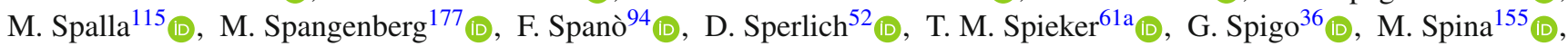

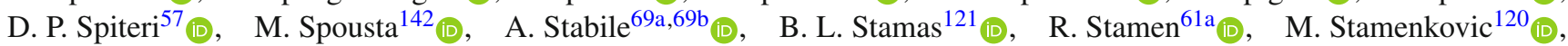

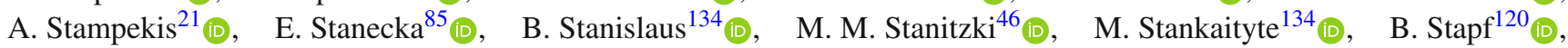

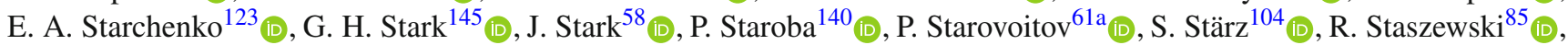

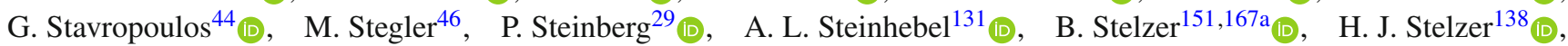

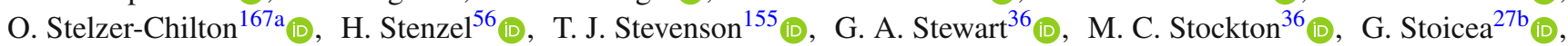

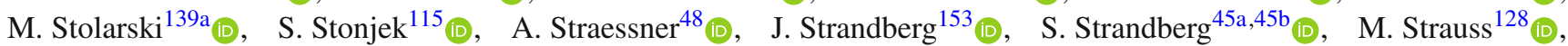

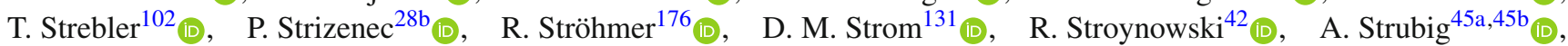

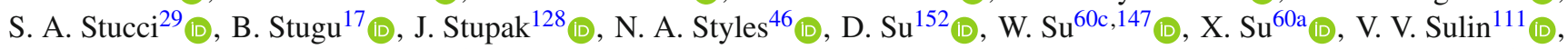

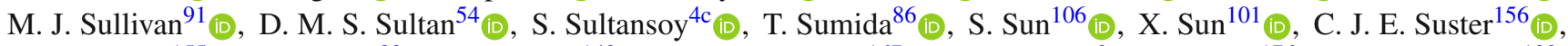

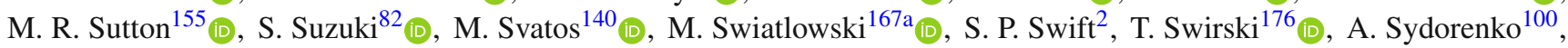

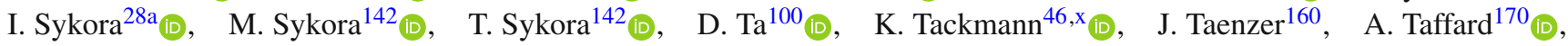
R. Tafirout ${ }^{167 a}$ (D), E. Tagiev ${ }^{123}$ (D), R. Takashima ${ }^{87}$, K. Takeda $^{83}$ (I) T. Takeshita $^{149}$ (D), E. P. Takeva ${ }^{50}$ (D), Y. Takubo ${ }^{82}$ (D), M. Talby ${ }^{102}$ (D) A. A. Talyshev ${ }^{122 a, 122 b}$, K. C. Tam ${ }^{63 b}$, N. M. Tamir ${ }^{160}$, J. Tanaka ${ }^{162}$ (D), R. Tanaka ${ }^{65}$ (D) S. Tapia Araya ${ }^{172}$ (D),

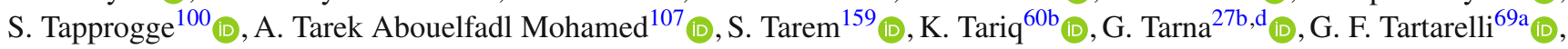

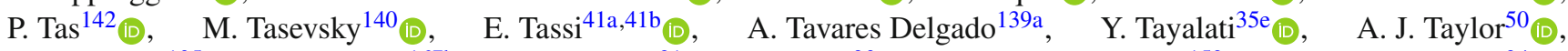
G. N. Taylor ${ }^{105}$ (D), W. Taylor ${ }^{167 b} \mathbb{C}_{0}, \quad$ H. Teagle ${ }^{91}, \quad$ A. S. Tee ${ }^{90}, \quad$ R. Teixeira De Lima ${ }^{152}$ (D), P. Teixeira-Dias ${ }^{94}$ (D),

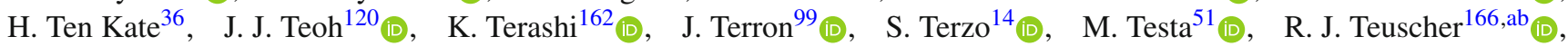
S. J. Thais ${ }^{182}$ (D, N. Themistokleous ${ }^{50}$ (i), T. Theveneaux-Pelzer ${ }^{46}$ (D) F. Thiele ${ }^{40}$ (D) D. W. Thomas ${ }^{94}$, J. O. Thomas ${ }^{42}$,

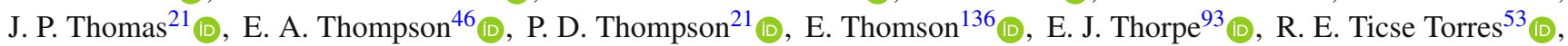

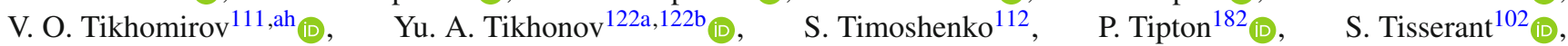

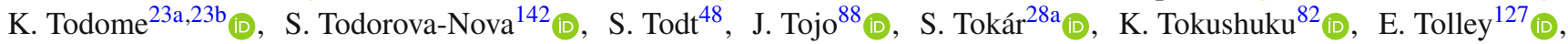

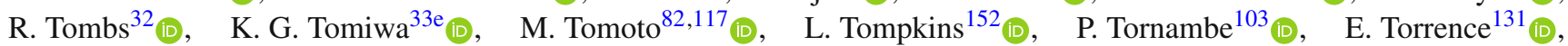

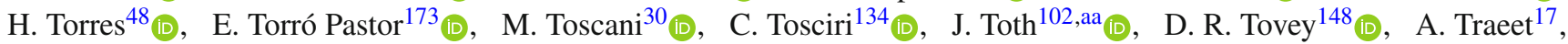

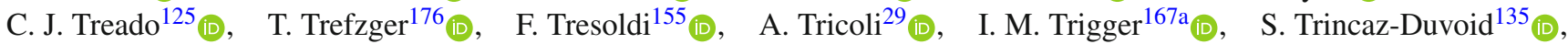

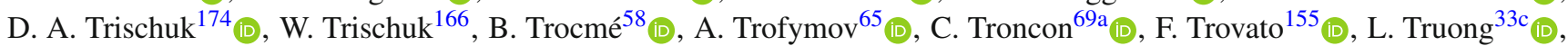

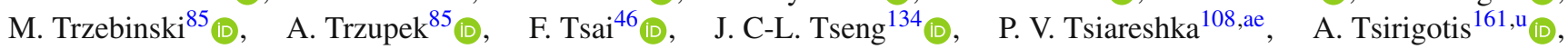
V. Tsiskaridze ${ }^{154}$ (D), E. G. Tskhadadze ${ }^{158 a}$, M. Tsopoulou ${ }^{161}$ (D), I. I. Tsukerman ${ }^{124}$ (D), V. Tsulaia ${ }^{18}$ (D), S. Tsuno ${ }^{82}$ (D),

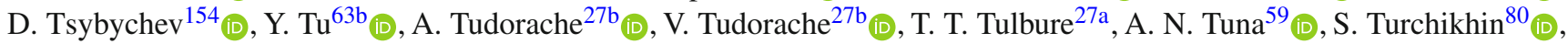

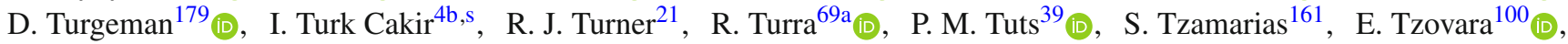

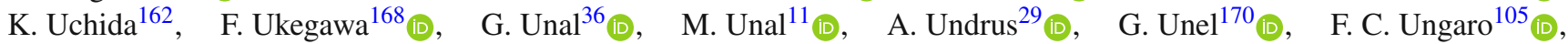

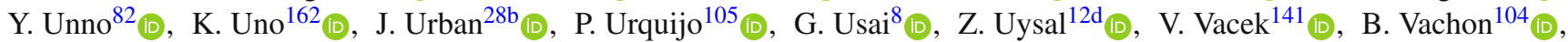

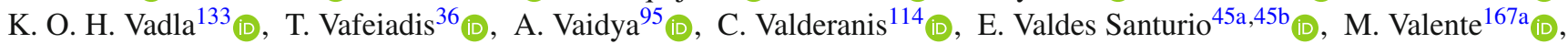

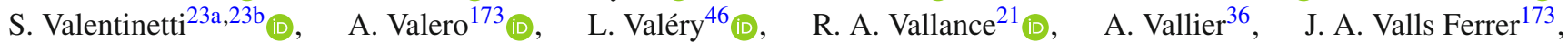

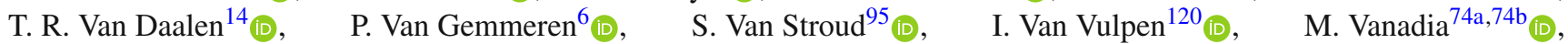
W. Vandelli ${ }^{36}$ (D), M. Vandenbroucke ${ }^{144}$ (D), E. R. Vandewall ${ }^{129}$ (D), A. Vaniachine ${ }^{165}$ (D), D. Vannicola ${ }^{73 a}, 73 b$ (D),

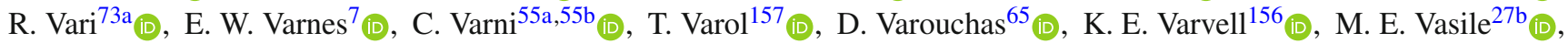


G. A. Vasquez ${ }^{175}$ (D), F. Vazeille ${ }^{38}$ (D), D. Vazquez Furelos ${ }^{14}$ (I), T. Vazquez Schroeder $^{36}$ (D), J. Veatch ${ }^{53}$ (D), V. Vecchio ${ }^{101}$ (D),

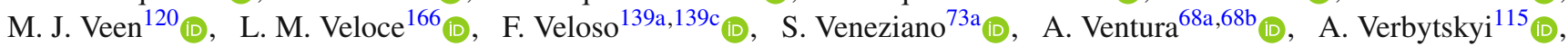

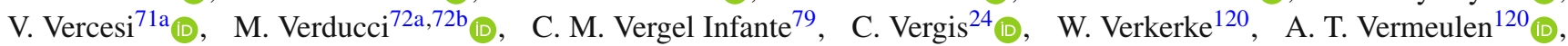

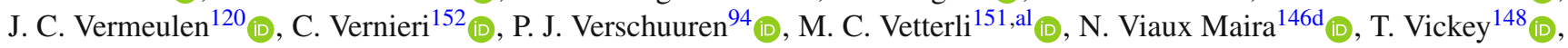

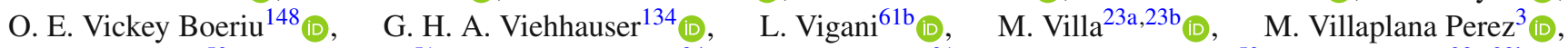

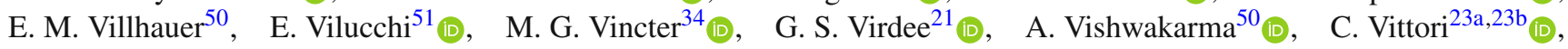

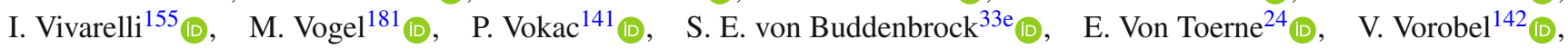

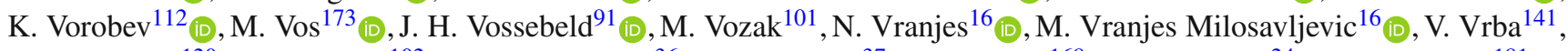
M. Vreeswijk ${ }^{120}$ (D) N. K. Vu ${ }^{102}$ (i), R. Vuillermet ${ }^{36}$ (D) I. Vukotic ${ }^{37}$ (D) S. Wada ${ }^{168}$ (D), P. Wagner ${ }^{24}$ (D), W. Wagner ${ }^{181}$ (D), J. Wagner-Kuhr ${ }^{114}$ (D), S. Wahdan ${ }^{181}$ (D), H. Wahlberg ${ }^{89}$ (D), R. Wakasa ${ }^{168}$ (D), V. M. Walbrecht ${ }^{115}$ (I),$\quad$ J. Walder ${ }^{143}$ (D),

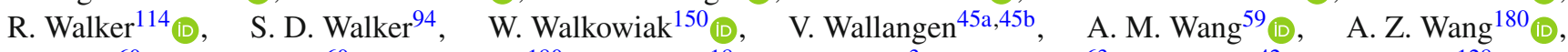

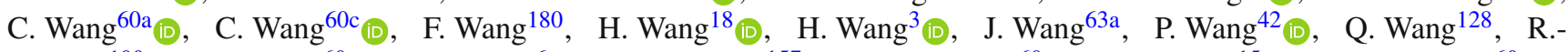

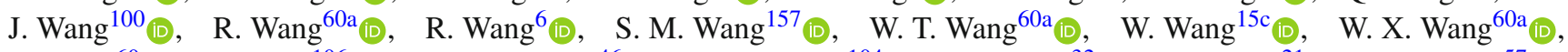

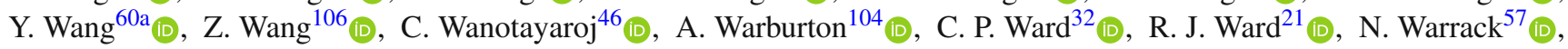
A. T. Watson ${ }^{21}$ (D) M. F. Watson ${ }^{21}$ (D), G. Watts ${ }^{147}$ (D), B. M. Waugh ${ }^{95}$ (D) A. F. Webb ${ }^{11}$ (D) C. Weber ${ }^{29}$ (D), M. S. Weber ${ }^{20}$ (D),

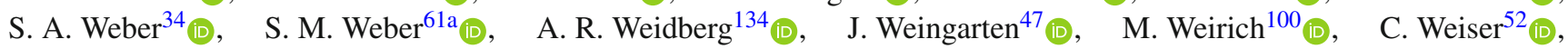

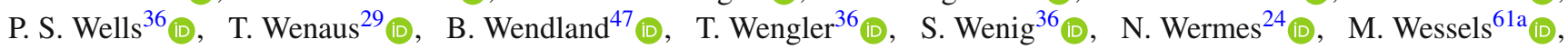

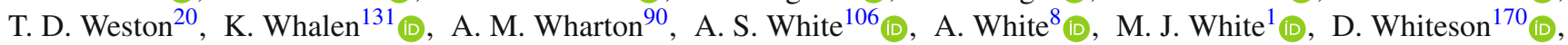
B. W. Whitmore ${ }^{90}$ (D), W. Wiedenmann ${ }^{180}$ (D), C. Wiel ${ }^{48}$ (D), M. Wielers ${ }^{143}$ (D), N. Wieseotte ${ }^{100}$, C. Wiglesworth ${ }^{40}$ (D), L. A. M. Wiik-Fuchs ${ }^{52}$ (D) H. G. Wilkens ${ }^{36}$ (D) L. J. Wilkins ${ }^{94}$ (D) H. H. Williams ${ }^{136}$, S. Williams ${ }^{32}$, S. Willocq ${ }^{103}$ (D), P. J. Windischhofer ${ }^{134}$ (D), I. Wingerter-Seez ${ }^{5}$, E. Winkels ${ }^{155}$ (D), F. Winklmeier ${ }^{131}$ (D), B. T. Winter ${ }^{52}$ (D), M. Wittgen ${ }^{152}$,

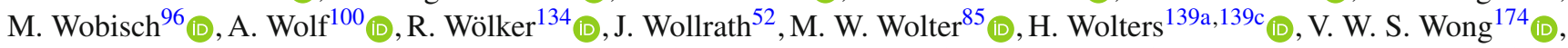

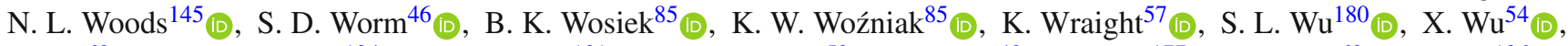

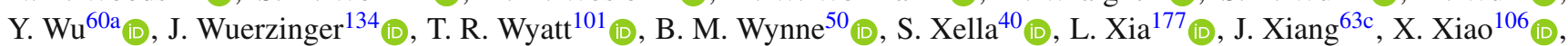

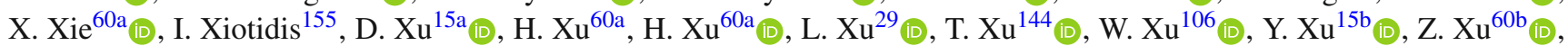

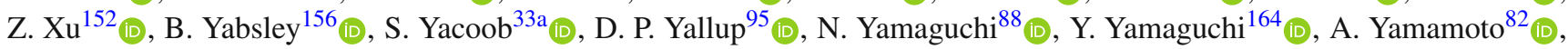

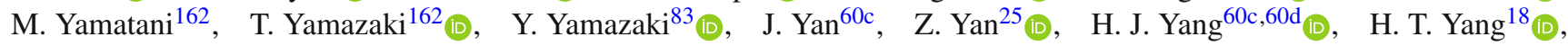

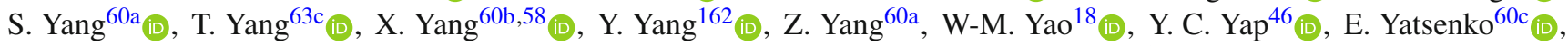

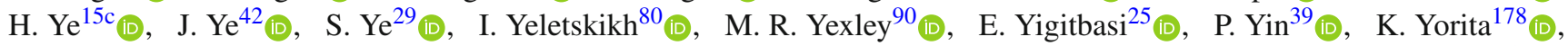

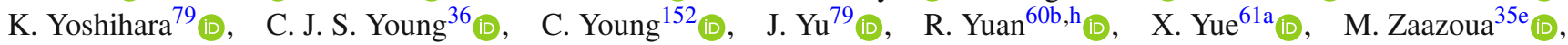

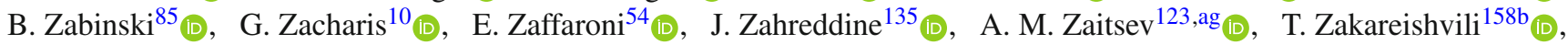
N. Zakharchuk ${ }^{34}$ (D) S. Zambito ${ }^{36}$ (D) D. Zanzi ${ }^{36}$ (D) S. V. Zeißner ${ }^{47}$ (D) C. Zeitnitz ${ }^{181}$ (D), G. Zemaityte ${ }^{134}$ (D), J. C. Zeng ${ }^{172}$ (D),

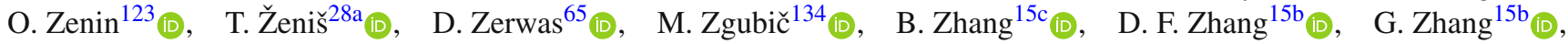

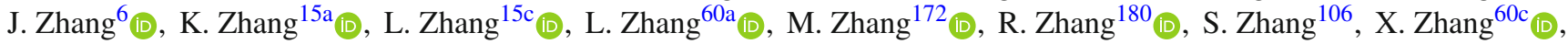

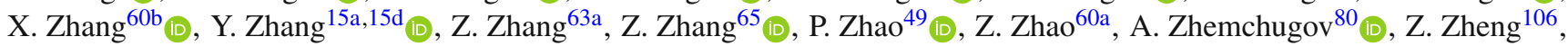

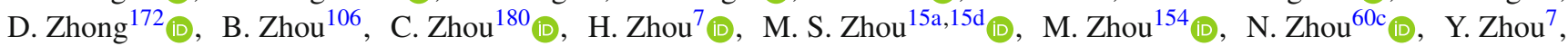

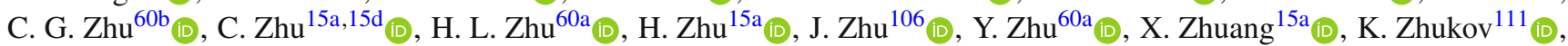

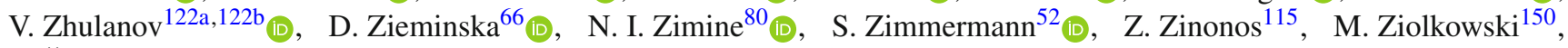

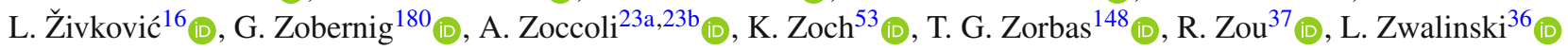

${ }^{1}$ Department of Physics, University of Adelaide, Adelaide, Australia

${ }^{2}$ Physics Department, SUNY Albany, Albany, NY, USA

${ }^{3}$ Department of Physics, University of Alberta, Edmonton, AB, Canada

4 (a) Department of Physics, Ankara University, Ankara, Turkey; ${ }^{(b)}$ Istanbul Aydin University, Application and Research Center for Advanced Studies, Istanbul, Turkey; ${ }^{(c)}$ Division of Physics, TOBB University of Economics and Technology, Ankara, Turkey

${ }^{5}$ LAPP, Université Grenoble Alpes, Université Savoie Mont Blanc, CNRS/IN2P3, Annecy, France

${ }^{6}$ High Energy Physics Division, Argonne National Laboratory, Argonne, IL, USA

${ }^{7}$ Department of Physics, University of Arizona, Tucson, AZ, USA

${ }^{8}$ Department of Physics, University of Texas at Arlington, Arlington, TX, USA

${ }^{9}$ Physics Department, National and Kapodistrian University of Athens, Athens, Greece

${ }^{10}$ Physics Department, National Technical University of Athens, Zografou, Greece

${ }^{11}$ Department of Physics, University of Texas at Austin, Austin, TX, USA 
12 (a) Faculty of Engineering and Natural Sciences, Bahcesehir University, Istanbul, Turkey; ${ }^{(b)}$ Faculty of Engineering and Natural Sciences, Istanbul Bilgi University, Istanbul, Turkey; ${ }^{(c)}$ Department of Physics, Bogazici University, Istanbul, Turkey; ${ }^{(d)}$ Department of Physics Engineering, Gaziantep University, Gaziantep, Turkey

${ }^{13}$ Institute of Physics, Azerbaijan Academy of Sciences, Baku, Azerbaijan

${ }^{14}$ Institut de Física d'Altes Energies (IFAE), Barcelona Institute of Science and Technology, Barcelona, Spain

15 (a) Institute of High Energy Physics, Chinese Academy of Sciences, Beijing, China; ${ }^{(b)}$ Physics Department, Tsinghua University, Beijing, China; ${ }^{(c)}$ Department of Physics, Nanjing University, Nanjing, China; ${ }^{(d)}$ University of Chinese Academy of Science (UCAS), Beijing, China

${ }^{16}$ Institute of Physics, University of Belgrade, Belgrade, Serbia

${ }^{17}$ Department for Physics and Technology, University of Bergen, Bergen, Norway

${ }^{18}$ Physics Division, Lawrence Berkeley National Laboratory and University of California, Berkeley, CA, USA

${ }^{19}$ Institut für Physik, Humboldt Universität zu Berlin, Berlin, Germany

${ }^{20}$ Albert Einstein Center for Fundamental Physics and Laboratory for High Energy Physics, University of Bern, Bern, Switzerland

${ }^{21}$ School of Physics and Astronomy, University of Birmingham, Birmingham, UK

22 (a) Facultad de Ciencias y Centro de Investigaciónes, Universidad Antonio Nariño, Bogotá, Colombia; ${ }^{(b)}$ Departamento de Física, Universidad Nacional de Colombia, Bogotá, Colombia

23 (a) Dipartimento di Fisica, INFN Bologna and Universita' di Bologna, Bologna, Italy; ${ }^{(b)}$ INFN Sezione di Bologna, Bologna, Italy

${ }^{24}$ Physikalisches Institut, Universität Bonn, Bonn, Germany

${ }^{25}$ Department of Physics, Boston University, Boston, MA, USA

${ }^{26}$ Department of Physics, Brandeis University, Waltham, MA, USA

27 (a) Transilvania University of Brasov, Brasov, Romania; ${ }^{(b)}$ Horia Hulubei National Institute of Physics and Nuclear Engineering, Bucharest, Romania; ${ }^{(c)}$ Department of Physics, Alexandru Ioan Cuza University of Iasi, Iasi,

Romania; ${ }^{(d)}$ Physics Department, National Institute for Research and Development of Isotopic and Molecular Technologies, Cluj-Napoca, Romania; ${ }^{(e)}$ University Politehnica Bucharest, Bucharest, Romania; ${ }^{(f)}$ West University in Timisoara, Timisoara, Romania

28 (a) Faculty of Mathematics, Physics and Informatics, Comenius University, Bratislava, Slovak Republic; (b) Department of Subnuclear Physics, Institute of Experimental Physics of the Slovak Academy of Sciences, Kosice, Slovak Republic

${ }^{29}$ Physics Department, Brookhaven National Laboratory, Upton, NY, USA

${ }^{30}$ Departamento de Física, Universidad de Buenos Aires, Buenos Aires, Argentina

${ }^{31}$ California State University, Long Beach, CA, USA

${ }^{32}$ Cavendish Laboratory, University of Cambridge, Cambridge, UK

33 (a) Department of Physics, University of Cape Town, Cape Town, South Africa; ${ }^{(b)}$ iThemba Labs, Western Cape, South Africa; ${ }^{(c)}$ Department of Mechanical Engineering Science, University of Johannesburg, Johannesburg, South Africa; ${ }^{(d)}$ Department of Physics, University of South Africa, Pretoria, South Africa; ${ }^{(e)}$ School of Physics, University of the Witwatersrand, Johannesburg, South Africa

${ }^{34}$ Department of Physics, Carleton University, Ottawa, ON, Canada

35 (a) Faculté des Sciences Ain Chock, Réseau Universitaire de Physique des Hautes Energies, Université Hassan II, Casablanca, Morocco; ${ }^{(b)}$ Faculté des Sciences, Université Ibn-Tofail, Kénitra, Morocco; ${ }^{(c)}$ Faculté des Sciences Semlalia, Université Cadi Ayyad, LPHEA, Marrakech, Morocco; ${ }^{(d)}$ Faculté des Sciences, Université Mohamed Premier and LPTPM, Oujda, Morocco; ${ }^{(\mathrm{e})}$ Faculté des sciences, Université Mohammed V, Rabat, Morocco

${ }^{36}$ CERN, Geneva, Switzerland

${ }^{37}$ Enrico Fermi Institute, University of Chicago, Chicago, IL, USA

${ }^{38}$ LPC, Université Clermont Auvergne, CNRS/IN2P3, Clermont-Ferrand, France

${ }^{39}$ Nevis Laboratory, Columbia University, Irvington, NY, USA

${ }^{40}$ Niels Bohr Institute, University of Copenhagen, Copenhagen, Denmark

41 (a) Dipartimento di Fisica, Università della Calabria, Rende, Italy; ${ }^{(b)}$ INFN Gruppo Collegato di Cosenza, Laboratori Nazionali di Frascati, Frascati, Italy

${ }^{42}$ Physics Department, Southern Methodist University, Dallas, TX, USA

${ }^{43}$ Physics Department, University of Texas at Dallas, Richardson, TX, USA

${ }^{44}$ National Centre for Scientific Research "Demokritos", Agia Paraskevi, Greece

45 (a) Department of Physics, Stockholm University, Stockholm, Sweden; ${ }^{(b)}$ Oskar Klein Centre, Stockholm, Sweden 
${ }^{46}$ Deutsches Elektronen-Synchrotron DESY, Hamburg and Zeuthen, Germany

${ }^{47}$ Lehrstuhl für Experimentelle Physik IV, Technische Universität Dortmund, Dortmund, Germany

${ }^{48}$ Institut für Kern- und Teilchenphysik, Technische Universität Dresden, Dresden, Germany

${ }^{49}$ Department of Physics, Duke University, Durham, NC, USA

${ }^{50}$ SUPA-School of Physics and Astronomy, University of Edinburgh, Edinburgh, UK

${ }^{51}$ INFN e Laboratori Nazionali di Frascati, Frascati, Italy

52 Physikalisches Institut, Albert-Ludwigs-Universität Freiburg, Freiburg, Germany

${ }^{53}$ II. Physikalisches Institut, Georg-August-Universität Göttingen, Göttingen, Germany

54 Département de Physique Nucléaire et Corpusculaire, Université de Genève, Geneva, Switzerland

55 (a) Dipartimento di Fisica, Università di Genova, Genoa, Italy; ${ }^{(b)}$ INFN Sezione di Genova, Genoa, Italy

${ }^{56}$ II. Physikalisches Institut, Justus-Liebig-Universität Giessen, Giessen, Germany

${ }^{57}$ SUPA-School of Physics and Astronomy, University of Glasgow, Glasgow, UK

${ }^{58}$ LPSC, Université Grenoble Alpes, CNRS/IN2P3, Grenoble INP, Grenoble, France

${ }^{59}$ Laboratory for Particle Physics and Cosmology, Harvard University, Cambridge, MA, USA

60 (a) Department of Modern Physics and State Key Laboratory of Particle Detection and Electronics, University of Science and Technology of China, Hefei, China; ${ }^{(b)}$ Institute of Frontier and Interdisciplinary Science and Key Laboratory of Particle Physics and Particle Irradiation (MOE), Shandong University, Qingdao, China; ${ }^{(c)}$ School of Physics and Astronomy, Shanghai Jiao Tong University, KLPPAC-MoE, SKLPPC, Shanghai, China; ${ }^{(d)}$ Tsung-Dao Lee Institute, Shanghai, China

61 (a) Kirchhoff-Institut für Physik, Ruprecht-Karls-Universität Heidelberg, Heidelberg, Germany; ${ }^{(b)}$ Physikalisches Institut, Ruprecht-Karls-Universität Heidelberg, Heidelberg, Germany

${ }^{62}$ Faculty of Applied Information Science, Hiroshima Institute of Technology, Hiroshima, Japan

63 (a) Department of Physics, Chinese University of Hong Kong, Shatin, N.T., Hong Kong, China; ${ }^{(b)}$ Department of Physics, University of Hong Kong, Hong Kong, China; ${ }^{\left({ }^{c}\right)}$ Department of Physics and Institute for Advanced Study, Hong Kong

University of Science and Technology, Clear Water Bay, Kowloon, Hong Kong, China

${ }^{64}$ Department of Physics, National Tsing Hua University, Hsinchu, Taiwan

${ }^{65}$ IJCLab, Université Paris-Saclay, CNRS/IN2P3, 91405 Orsay, France

${ }^{66}$ Department of Physics, Indiana University, Bloomington, IN, USA

67 (a) INFN Gruppo Collegato di Udine, Sezione di Trieste, Udine, Italy; ${ }^{(b)}$ ICTP, Trieste, Italy; ${ }^{(c)}$ Dipartimento Politecnico di Ingegneria e Architettura, Università di Udine, Udine, Italy

68 (a) INFN Sezione di Lecce, Lecce, Italy; ${ }^{\left({ }^{b}\right)}$ Dipartimento di Matematica e Fisica, Università del Salento, Lecce, Italy

69 (a) INFN Sezione di Milano, Milan, Italy; ${ }^{(b)}$ Dipartimento di Fisica, Università di Milano, Milan, Italy

70 (a) INFN Sezione di Napoli, Naples, Italy; ${ }^{(b)}$ Dipartimento di Fisica, Università di Napoli, Naples, Italy

71 (a) INFN Sezione di Pavia, Pavia, Italy; ${ }^{(b)}$ Dipartimento di Fisica, Università di Pavia, Pavia, Italy

72 (a) INFN Sezione di Pisa, Pisa, Italy; ${ }^{\text {(b) }}$ Dipartimento di Fisica E. Fermi, Università di Pisa, Pisa, Italy

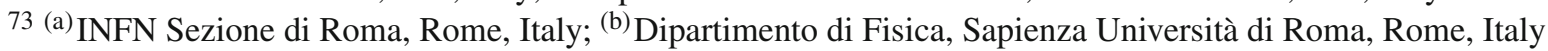

74 (a) INFN Sezione di Roma Tor Vergata, Rome, Italy; ${ }^{(b)}$ Dipartimento di Fisica, Università di Roma Tor Vergata, Rome, Italy

75 (a) INFN Sezione di Roma Tre, Rome, Italy; ${ }^{(b)}$ Dipartimento di Matematica e Fisica, Università Roma Tre, Rome, Italy

76 (a) INFN-TIFPA, Trento, Italy; (b) Università degli Studi di Trento, Trento, Italy

${ }^{77}$ Institut für Astro- und Teilchenphysik, Leopold-Franzens-Universität, Innsbruck, Austria

${ }^{78}$ University of Iowa, Iowa City, IA, USA

${ }^{79}$ Department of Physics and Astronomy, Iowa State University, Ames, IA, USA

${ }^{80}$ Joint Institute for Nuclear Research, Dubna, Russia

81 (a) Departamento de Engenharia Elétrica, Universidade Federal de Juiz de Fora (UFJF), Juiz de Fora,

Brazil; ${ }^{(b)}$ Universidade Federal do Rio De Janeiro COPPE/EE/IF, Rio de Janeiro, Brazil; ${ }^{\left({ }^{c}\right)}$ Universidade Federal de São João del Rei (UFSJ), São João del Rei, Brazil; ${ }^{(d)}$ Instituto de Física, Universidade de São Paulo, São Paulo, Brazil

${ }^{82}$ KEK, High Energy Accelerator Research Organization, Tsukuba, Japan

${ }^{83}$ Graduate School of Science, Kobe University, Kobe, Japan

84 (a) Faculty of Physics and Applied Computer Science, AGH University of Science and Technology, Krakow,

Poland; ${ }^{(b)}$ Marian Smoluchowski Institute of Physics, Jagiellonian University, Krakow, Poland

${ }^{85}$ Institute of Nuclear Physics Polish Academy of Sciences, Krakow, Poland

${ }^{86}$ Faculty of Science, Kyoto University, Kyoto, Japan 
${ }^{87}$ Kyoto University of Education, Kyoto, Japan

${ }^{88}$ Research Center for Advanced Particle Physics and Department of Physics, Kyushu University, Fukuoka, Japan

${ }^{89}$ Instituto de Física La Plata, Universidad Nacional de La Plata and CONICET, La Plata, Argentina

${ }^{90}$ Physics Department, Lancaster University, Lancaster, UK

${ }^{91}$ Oliver Lodge Laboratory, University of Liverpool, Liverpool, UK

${ }^{92}$ Department of Experimental Particle Physics, Jožef Stefan Institute and Department of Physics, University of Ljubljana, Ljubljana, Slovenia

${ }^{93}$ School of Physics and Astronomy, Queen Mary University of London, London, UK

${ }^{94}$ Department of Physics, Royal Holloway University of London, Egham, UK

${ }^{95}$ Department of Physics and Astronomy, University College London, London, UK

${ }^{96}$ Louisiana Tech University, Ruston, LA, USA

${ }^{97}$ Fysiska institutionen, Lunds universitet, Lund, Sweden

${ }^{98}$ Centre de Calcul de l'Institut National de Physique Nucléaire et de Physique des Particules (IN2P3), Villeurbanne,

France

${ }^{99}$ Departamento de Física Teorica C-15 and CIAFF, Universidad Autónoma de Madrid, Madrid, Spain

${ }^{100}$ Institut für Physik, Universität Mainz, Mainz, Germany

${ }^{101}$ School of Physics and Astronomy, University of Manchester, Manchester, UK

102 CPPM, Aix-Marseille Université, CNRS/IN2P3, Marseille, France

${ }^{103}$ Department of Physics, University of Massachusetts, Amherst, MA, USA

104 Department of Physics, McGill University, Montreal, QC, Canada

105 School of Physics, University of Melbourne, Melbourne, VIC, Australia

${ }^{106}$ Department of Physics, University of Michigan, Ann Arbor, MI, USA

107 Department of Physics and Astronomy, Michigan State University, East Lansing, MI, USA

108 B.I. Stepanov Institute of Physics, National Academy of Sciences of Belarus, Minsk, Belarus

109 Research Institute for Nuclear Problems of Byelorussian State University, Minsk, Belarus

${ }^{110}$ Group of Particle Physics, University of Montreal, Montreal, QC, Canada

${ }^{111}$ P.N. Lebedev Physical Institute of the Russian Academy of Sciences, Moscow, Russia

${ }^{112}$ National Research Nuclear University MEPhI, Moscow, Russia

113 D.V. Skobeltsyn Institute of Nuclear Physics, M.V. Lomonosov Moscow State University, Moscow, Russia

${ }^{114}$ Fakultät für Physik, Ludwig-Maximilians-Universität München, Munich, Germany

115 Max-Planck-Institut für Physik (Werner-Heisenberg-Institut), Munich, Germany

${ }^{116}$ Nagasaki Institute of Applied Science, Nagasaki, Japan

117 Graduate School of Science and Kobayashi-Maskawa Institute, Nagoya University, Nagoya, Japan

118 Department of Physics and Astronomy, University of New Mexico, Albuquerque, NM, USA

${ }^{119}$ Institute for Mathematics, Astrophysics and Particle Physics, Radboud University Nijmegen/Nikhef, Nijmegen, The Netherlands

${ }^{120}$ Nikhef National Institute for Subatomic Physics and University of Amsterdam, Amsterdam, The Netherlands

121 Department of Physics, Northern Illinois University, DeKalb, IL, USA

122 (a) Budker Institute of Nuclear Physics and NSU, SB RAS, Novosibirsk, Russia; ${ }^{(b)}$ Novosibirsk State University

Novosibirsk, Novosibirsk, Russia

${ }^{123}$ Institute for High Energy Physics of the National Research Centre Kurchatov Institute, Protvino, Russia

${ }^{124}$ Institute for Theoretical and Experimental Physics named by A.I. Alikhanov of National Research Centre "Kurchatov Institute", Moscow, Russia

${ }^{125}$ Department of Physics, New York University, New York, NY, USA

${ }^{126}$ Ochanomizu University, Otsuka, Bunkyo-ku, Tokyo, Japan

127 Ohio State University, Columbus, OH, USA

${ }^{128}$ Homer L. Dodge Department of Physics and Astronomy, University of Oklahoma, Norman, OK, USA

${ }^{129}$ Department of Physics, Oklahoma State University, Stillwater, OK, USA

${ }^{130}$ Palacký University, RCPTM, Joint Laboratory of Optics, Olomouc, Czech Republic

${ }^{131}$ Institute for Fundamental Science, University of Oregon, Eugene, OR, USA

${ }^{132}$ Graduate School of Science, Osaka University, Osaka, Japan

${ }^{133}$ Department of Physics, University of Oslo, Oslo, Norway

${ }^{134}$ Department of Physics, Oxford University, Oxford, UK 
${ }^{135}$ LPNHE, Sorbonne Université, Université de Paris, CNRS/IN2P3, Paris, France

136 Department of Physics, University of Pennsylvania, Philadelphia, PA, USA

${ }^{137}$ Konstantinov Nuclear Physics Institute of National Research Centre "Kurchatov Institute", PNPI, St. Petersburg, Russia

138 Department of Physics and Astronomy, University of Pittsburgh, Pittsburgh, PA, USA

139 (a) Laboratório de Instrumentação e Física Experimental de Partículas - LIP, Lisbon, Portugal; ${ }^{(b)}$ Departamento de Física, Faculdade de Ciências, Universidade de Lisboa, Lisbon, Portugal; ${ }^{\left({ }^{c}\right)}$ Departamento de Física, Universidade de Coimbra, Coimbra, Portugal; ${ }^{(d)}$ Centro de Física Nuclear da Universidade de Lisboa, Lisbon, Portugal; ${ }^{(e)}$ Departamento de Física, Universidade do Minho, Braga, Portugal; ${ }^{(f)}$ Departamento de Física Teórica y del Cosmos, Universidad de Granada, Granada, Spain; ${ }^{(\mathrm{g})}$ Dep Física and CEFITEC of Faculdade de Ciências e Tecnologia, Universidade Nova de Lisboa, Caparica, Portugal; ${ }^{(\mathrm{h})}$ Instituto Superior Técnico, Universidade de Lisboa, Lisbon, Portugal

${ }^{140}$ Institute of Physics of the Czech Academy of Sciences, Prague, Czech Republic

${ }^{141}$ Czech Technical University in Prague, Prague, Czech Republic

${ }^{142}$ Faculty of Mathematics and Physics, Charles University, Prague, Czech Republic

${ }^{143}$ Particle Physics Department, Rutherford Appleton Laboratory, Didcot, UK

${ }^{144}$ IRFU, CEA, Université Paris-Saclay, Gif-sur-Yvette, France

${ }^{145}$ Santa Cruz Institute for Particle Physics, University of California Santa Cruz, Santa Cruz, CA, USA

146 (a) Departamento de Física, Pontificia Universidad Católica de Chile, Santiago, Chile; ${ }^{\left({ }^{b}\right)}$ Department of Physics,

Universidad Andres Bello, Santiago, Chile; ( ${ }^{(c)}$ Instituto de Alta Investigación, Universidad de Tarapacá, Arica,

Chile; ${ }^{(d)}$ Departamento de Física, Universidad Técnica Federico Santa María, Valparaíso, Chile

${ }^{147}$ Department of Physics, University of Washington, Seattle, WA, USA

${ }^{148}$ Department of Physics and Astronomy, University of Sheffield, Sheffield, UK

${ }^{149}$ Department of Physics, Shinshu University, Nagano, Japan

${ }^{150}$ Department Physik, Universität Siegen, Siegen, Germany

${ }^{151}$ Department of Physics, Simon Fraser University, Burnaby, BC, Canada

${ }^{152}$ SLAC National Accelerator Laboratory, Stanford, CA, USA

153 Physics Department, Royal Institute of Technology, Stockholm, Sweden

${ }^{154}$ Departments of Physics and Astronomy, Stony Brook University, Stony Brook, NY, USA

155 Department of Physics and Astronomy, University of Sussex, Brighton, UK

${ }^{156}$ School of Physics, University of Sydney, Sydney, Australia

${ }^{157}$ Institute of Physics, Academia Sinica, Taipei, Taiwan

158 (a) E. Andronikashvili Institute of Physics, Iv. Javakhishvili Tbilisi State University, Tbilisi, Georgia; ${ }^{(b)}$ High Energy

Physics Institute, Tbilisi State University, Tbilisi, Georgia

${ }^{159}$ Department of Physics, Technion, Israel Institute of Technology, Haifa, Israel

${ }^{160}$ Raymond and Beverly Sackler School of Physics and Astronomy, Tel Aviv University, Tel Aviv, Israel

${ }^{161}$ Department of Physics, Aristotle University of Thessaloniki, Thessaloniki, Greece

${ }^{162}$ International Center for Elementary Particle Physics and Department of Physics, University of Tokyo, Tokyo, Japan

${ }^{163}$ Graduate School of Science and Technology, Tokyo Metropolitan University, Tokyo, Japan

${ }^{164}$ Department of Physics, Tokyo Institute of Technology, Tokyo, Japan

165 Tomsk State University, Tomsk, Russia

${ }^{166}$ Department of Physics, University of Toronto, Toronto, ON, Canada

167 (a) TRIUMF, Vancouver, BC, Canada; ${ }^{(b)}$ Department of Physics and Astronomy, York University, Toronto, ON, Canada

168 Division of Physics and Tomonaga Center for the History of the Universe, Faculty of Pure and Applied Sciences, University of Tsukuba, Tsukuba, Japan

${ }^{169}$ Department of Physics and Astronomy, Tufts University, Medford, MA, USA

${ }^{170}$ Department of Physics and Astronomy, University of California Irvine, Irvine, CA, USA

${ }^{171}$ Department of Physics and Astronomy, University of Uppsala, Uppsala, Sweden

${ }^{172}$ Department of Physics, University of Illinois, Urbana, IL, USA

${ }^{173}$ Instituto de Física Corpuscular (IFIC), Centro Mixto Universidad de Valencia - CSIC, Valencia, Spain

${ }^{174}$ Department of Physics, University of British Columbia, Vancouver, BC, Canada

175 Department of Physics and Astronomy, University of Victoria, Victoria, BC, Canada

${ }^{176}$ Fakultät für Physik und Astronomie, Julius-Maximilians-Universität Würzburg, Würzburg, Germany

177 Department of Physics, University of Warwick, Coventry, UK

178 Waseda University, Tokyo, Japan 
179 Department of Particle Physics and Astrophysics, Weizmann Institute of Science, Rehovot, Israel

${ }^{180}$ Department of Physics, University of Wisconsin, Madison, WI, USA

${ }^{181}$ Fakultät für Mathematik und Naturwissenschaften, Fachgruppe Physik, Bergische Universität Wuppertal, Wuppertal, Germany

182 Department of Physics, Yale University, New Haven, CT, USA

${ }^{a}$ Also at Borough of Manhattan Community College, City University of New York, New York, NY, USA

b Also at Centro Studi e Ricerche Enrico Fermi, Roma, Italy

${ }^{c}$ Also at CERN, Geneva, Switzerland

${ }^{d}$ Also at CPPM, Aix-Marseille Université, CNRS/IN2P3, Marseille, France

e Also at Département de Physique Nucléaire et Corpusculaire, Université de Genève, Geneva, Switzerland

${ }^{\mathrm{f}}$ Also at Departament de Fisica de la Universitat Autonoma de Barcelona, Barcelona, Spain

g Also at Department of Financial and Management Engineering, University of the Aegean, Chios, Greece

${ }^{\mathrm{h}}$ Also at Department of Physics and Astronomy, Michigan State University, East Lansing, MI, USA

${ }^{\mathrm{i}}$ Also at Department of Physics and Astronomy, University of Louisville, Louisville, KY, USA

j Also at Department of Physics, Ben Gurion University of the Negev, Beer Sheva, Israel

${ }^{\mathrm{k}}$ Also at Department of Physics, California State University, East Bay, USA

${ }^{1}$ Also at Department of Physics, California State University, Fresno, USA

m Also at Department of Physics, California State University, Sacramento, USA

${ }^{n}$ Also at Department of Physics, King's College London, London, UK

${ }^{\circ}$ Also at Department of Physics, St. Petersburg State Polytechnical University, St. Petersburg, Russia

p Also at Department of Physics, University of Fribourg, Fribourg, Switzerland

${ }^{\mathrm{q}}$ Also at Dipartimento di Matematica, Informatica e Fisica, Università di Udine, Udine, Italy

${ }^{r}$ Also at Faculty of Physics, M.V. Lomonosov Moscow State University, Moscow, Russia

${ }^{s}$ Also at Giresun University, Faculty of Engineering, Giresun, Turkey

${ }^{t}$ Also at Graduate School of Science, Osaka University, Osaka, Japan

"Also at Hellenic Open University, Patras, Greece

v Also at IJCLab, Université Paris-Saclay, CNRS/IN2P3, 91405, Orsay, France

${ }^{\text {w }}$ Also at Institucio Catalana de Recerca i Estudis Avancats, ICREA, Barcelona, Spain

${ }^{x}$ Also at Institut für Experimentalphysik, Universität Hamburg, Hamburg, Germany

${ }^{y}$ Also at Institute for Mathematics, Astrophysics and Particle Physics, Radboud University Nijmegen/Nikhef, Nijmegen, The Netherlands

${ }^{\mathrm{z}}$ Also at Institute for Nuclear Research and Nuclear Energy (INRNE) of the Bulgarian Academy of Sciences, Sofia, Bulgaria

aa Also at Institute for Particle and Nuclear Physics, Wigner Research Centre for Physics, Budapest, Hungary

${ }^{\text {ab }}$ Also at Institute of Particle Physics (IPP), Montreal, Canada

${ }^{\text {ac }}$ Also at Institute of Physics, Azerbaijan Academy of Sciences, Baku, Azerbaijan

ad Also at Instituto de Fisica Teorica, IFT-UAM/CSIC, Madrid, Spain

ae Also at Joint Institute for Nuclear Research, Dubna, Russia

${ }^{a}$ Also at Louisiana Tech University, Ruston, LA, USA

ag Also at Moscow Institute of Physics and Technology State University, Dolgoprudny, Russia

ah Also at National Research Nuclear University MEPhI, Moscow, Russia

ai Also at Physics Department, An-Najah National University, Nablus, Palestine

aj Also at Physikalisches Institut, Albert-Ludwigs-Universität Freiburg, Freiburg, Germany

ak Also at The City College of New York, New York, NY, USA

${ }^{\text {al }}$ Also at TRIUMF, Vancouver, BC, Canada

am Also at Universita di Napoli Parthenope, Naples, Italy

${ }^{\text {an }}$ Also at University of Chinese Academy of Sciences (UCAS), Beijing, China

ao $*$ Deceased 